NOTE TO USERS

This reproduction is the best copy available.

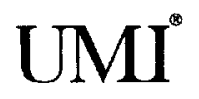





\title{
THE EFFECT OF POSTERIOR CAPSULAR PLICATION ON SUBACROMIAL PRESSURE DURING SIMULATED ACTIVE ABDUCTION IN THE SCAPULAR PLANE
}

By

\author{
Philippe Poitras
}

A thesis submitted to the Department of Biomedical

Engineering in conformity with the requirements

For the degree of Master of Applied Science

\author{
Carleton University \\ Ottawa, Ontario \\ June 30, 2009
}

Copyright ${ }^{\circ}$ Philippe Poitras, 2009 
Library and Archives

Canada

Published Heritage

Branch

395 Wellington Street

Ottawa ON K1A ON4

Canada
Bibliothèque et

Archives Canada

Direction du

Patrimoine de l'édition

395, rue Wellington

Ottawa ON K1A ON4

Canada
Your file Votre référence

ISBN: 978-0-494-60226-3

Our file Notre référence

ISBN: 978-0-494-60226-3
NOTICE:

The author has granted a nonexclusive license allowing Library and Archives Canada to reproduce, publish, archive, preserve, conserve, communicate to the public by telecommunication or on the Internet, loan, distribute and sell theses worldwide, for commercial or noncommercial purposes, in microform, paper, electronic and/or any other formats.

The author retains copyright ownership and moral rights in this thesis. Neither the thesis nor substantial extracts from it may be printed or otherwise reproduced without the author's permission.

\begin{abstract}
AVIS:
L'auteur a accordé une licence non exclusive permettant à la Bibliothèque et Archives Canada de reproduire, publier, archiver, sauvegarder, conserver, transmettre au public par télécommunication ou par l'Internet, prêter, distribuer et vendre des thèses partout dans le monde, à des fins commerciales ou autres, sur support microforme, papier, électronique et/ou autres formats.
\end{abstract}

L'auteur conserve la propriété du droit d'auteur et des droits moraux qui protège cette thèse. $\mathrm{Ni}$ la thèse ni des extraits substantiels de celle-ci ne doivent être imprimés ou autrement reproduits sans son autorisation.
In compliance with the Canadian Privacy Act some supporting forms may have been removed from this thesis.

While these forms may be included in the document page count, their removal does not represent any loss of content from the thesis.
Conformément à la loi canadienne sur la protection de la vie privée, quelques formulaires secondaires ont été enlevés de cette thèse.

Bien que ces formulaires aient inclus dans la pagination, il n'y aura aucun contenu manquant. 
THE EFFECT OF POSTERIOR CAPSULAR PLICATION ON SUBACROMIAL PRESSURE DURING SIMULATED ACTIVE ABDUCTION IN THE SCAPULAR PLANE

PHILIPPE POITRAS

2009 


\section{Abstract}

Subacromial impingement (SI) syndrome is a painful condition that occurs during overhead activities as the rotator cuff is compressed in the subacromial space. Posterior capsular tightness is often cited as a secondary cause of SI, however scientific evidence is lacking. The primary objective of this study was to evaluate the effect of posterior capsular plication on peak subacromial pressure during simulated active abduction in the scapular plane.

Ten fresh-frozen human cadaver shoulder specimens were mounted on a custom designed shoulder simulator. The deltoid and rotator cuff muscles were loaded to elevate the humerus in the scapular plane. For each treatment $(0 \mathrm{~cm}, 1 \mathrm{~cm}$ and $2 \mathrm{~cm}$ plication of the posterior capsule), contact pressure and glenohumeral kinematics were recorded.

Posterior capsular plication did not significantly increase subacromial pressure during abduction in the scapular plane. A future study simulating active flexion is necessary to fully characterize the contribution of posterior capsular tightness in SI. 


\section{Acknowledgments}

To Donald Russell, my supervisor, thank you for your continued support and encouragements. Your problem solving skills and engineering knowledge are second to none.

To Hans K. Uhthoff, my mentor, your wisdom, passion for research and relentless efforts to further understand our knowledge of the musculoskeletal system is inspiring. It has been my privilege to work with you. I am forever indebted to you for the countless hours we have spent together brainstorming and sharing ideas as well as for the lessons learned both in research and life.

To Peter Lapner, my colleague and friend, our research collaboration started eight years ago spending one full day a week at the Anatomy Laboratory working on the DRUJ project. One that is based on trust, understanding and mutual respect, I hope that we can continue our research collaboration for many years to come. Thank you for your support.

To David Backman, thank you for giving me my first opportunity at the Orthopaedic Biomechanics Laboratory. I will always remember your continued support and encouragement as well as your genuine enthusiasm for research.

To Nanthan Ramachandran, my colleague and friend, many thanks for your initial work on the design of the fixtures for the shoulder simulator. To Andrew Speirs thank you for all your help throughout the latter stages of this project. To both of you, many thanks for your patience and support.

To Stephen Kingwell and to Othman Ramadan, orthopaedic residents, I thoroughly enjoyed working with both of you. Stephen, you were instrumental during the 
grant writing process and Othman thank you for the numerous hours spent at the Anatomy Laboratory.

Finally, to Geoffrey F. Dervin and Alan Giachino as well as to all my colleagues at the Division of Orthopaedic Surgery, I am forever grateful for your continued support. 


\section{Table of Contents}

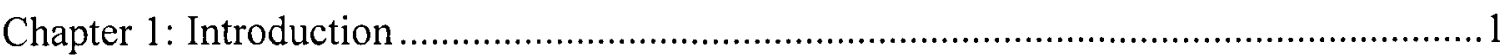

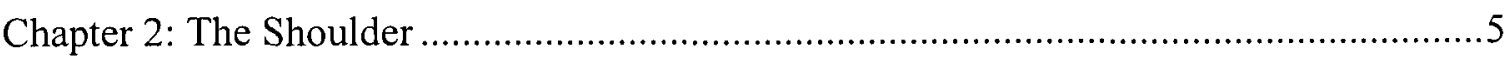

2.1 Anatomy and Biomechanics of the Glenohumeral Joint ........................................5

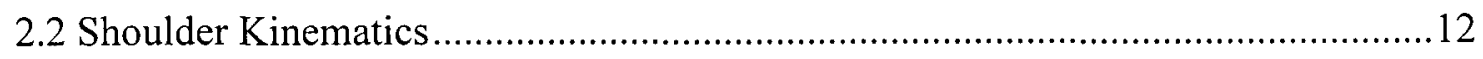

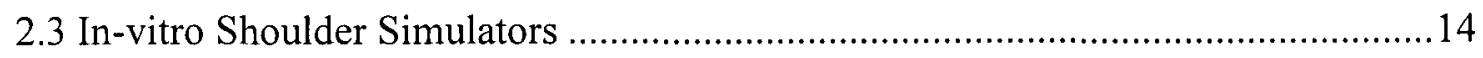

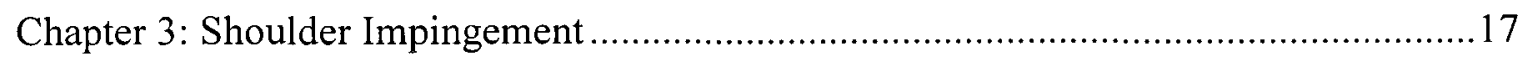

3.1 Definition, Diagnosis and Treatment.................................................................17

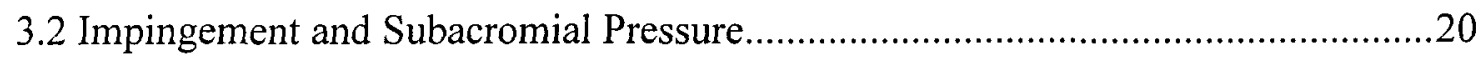

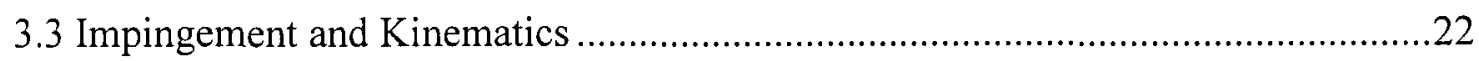

3.4 Role of Posterior Capsule in Shoulder Impingement ...........................................24

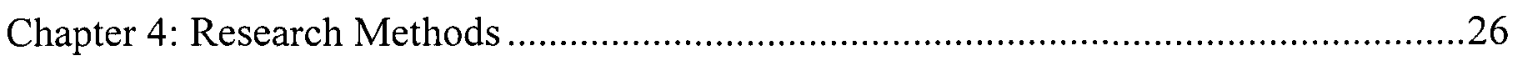

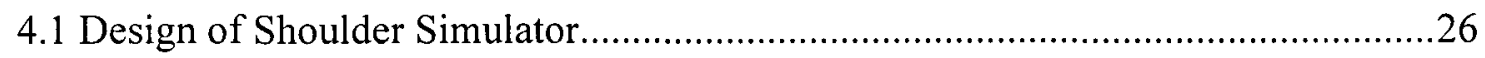

4.2 Measurement of Subacromial Pressure..............................................................34

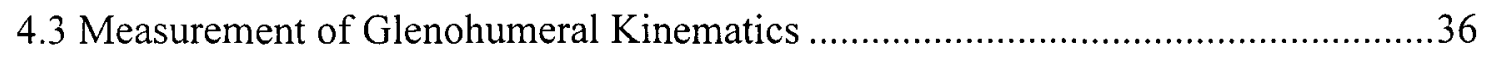

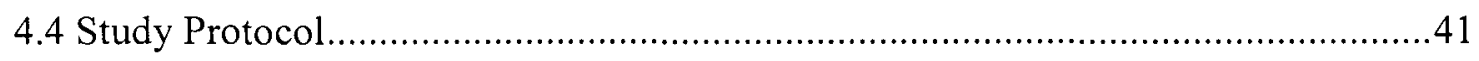

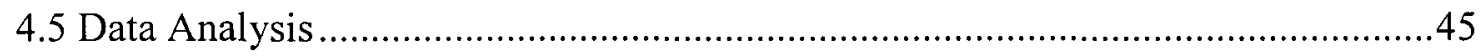

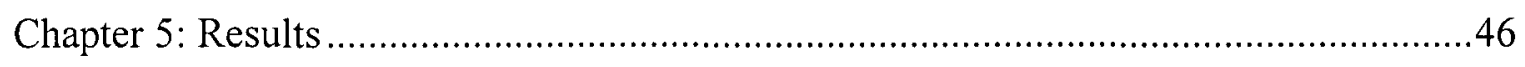

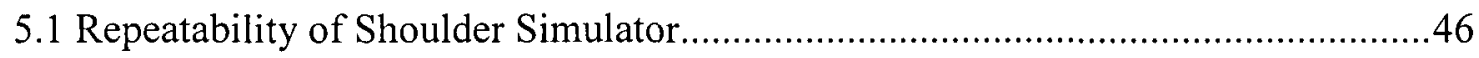

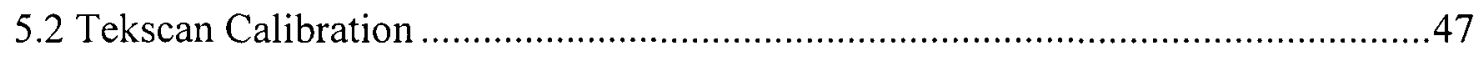

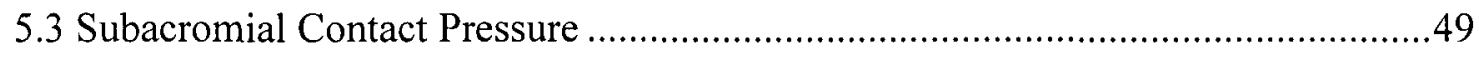

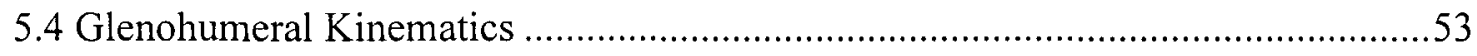

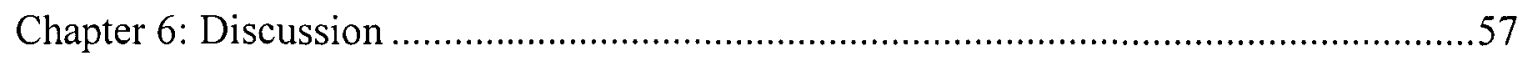




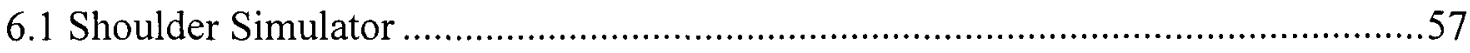

6.2 Subacromial Contact Pressure ........................................................61

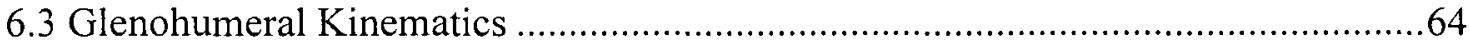

6.4 Effect of Posterior Capsular Plication .......................................................67

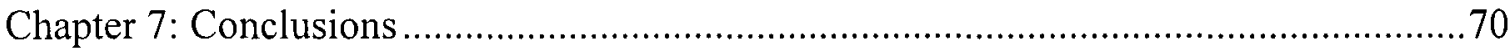

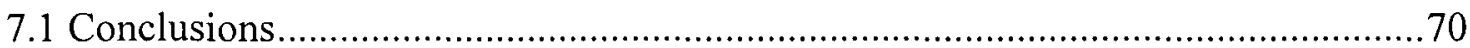

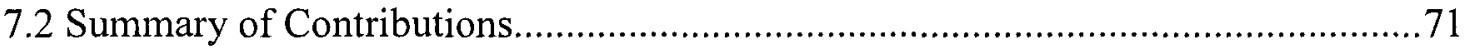

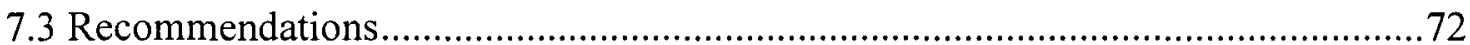

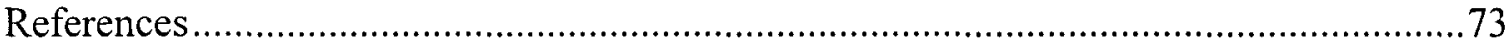

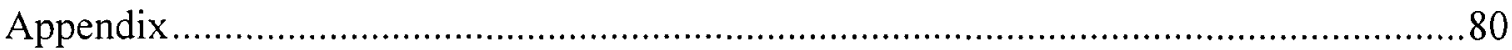




\section{List of Figures}

Figure 1: The shoulder is composed of the glenohumeral $(\mathrm{GH})$, acromioclavicular $(\mathrm{AC})$ and sternoclavicular joints. ${ }^{16}$

Figure 2: Medial and Posterior Offset of Center of Humeral Head. ${ }^{18}$................................ 6

Figure 3: (a) pear-shaped profile of glenoid (lateral view) ${ }^{19}$ and (b) retroversion and vertical tilt of glenoid. ${ }^{20}$ 7

Figure 4: Lateral cross-section of glenohumeral joint showing coracoacomial arch (arrow) which spans from the posterior aspect of the acromion to the coracoid process. ${ }^{23}$ 8

Figure 5: Ligaments at the glenohumeral joint: glenohumeral ligaments (capsular ligament), coraco-acromial ligament and coracohumeral ligament. ${ }^{25}$ 9

Figure 6: Rotator cuff (SSP, ISP, TM and SUB) and deltoid act to provide great range of motion and stabilize the glenohumeral joint. ${ }^{26}$ 10

Figure 7: Anterior, middle and posterior deltoids. ${ }^{27}$ 11

Figure 8: Forward flexion (A) and extension (B), abduction (C) ………………......... 12

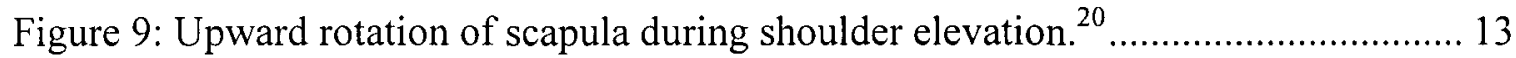

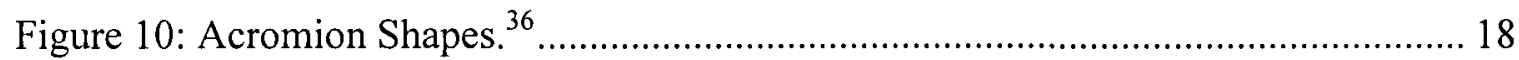

Figure 11: Schematic Representation of shoulder simulator.......................................... 27

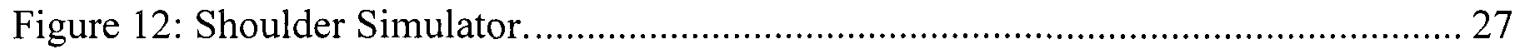

Figure 13: Alignment of Potting Cup in Shoulder Simulator with X-Y table................. 29

Figure 14: Deltoid cable activated by MTS load frame. Superior-inferior (SI) and mediolateral (ML) adjustment completed with slots. 
Figure 15: Static weights applied to rotator cuff: Supraspinatus (SSP), Subscapularis

(SUB) and Infraspinatus (ISP).

Figure 16: Rotator cuff static loading - effective gear ratio.

Figure 17: Adjustable eyelets align the rotator cuff cables with physiological lines of action. 33

Figure 18: Tekscan Calibration Fixtures..................................................................... 35

Figure 19: Definitions of Scapula and Humerus Coordinate Systems. ............................. 37

Figure 20: Schematic representation of posterior capsular plication............................... 42

Figure 21: Calibration curves $\left(y=A x^{b}\right)$ plotted for 150psi sensors............................... 48

Figure 22: Typical subacromial pressure map............................................................ 49

Figure 23: Peak subacromial contact pressure versus glenohumeral abduction angle for each specimen (pre-test data). ${ }^{*}$ subacromial contact pressure after 60 degrees reached saturation due to abnormal bump on greater tubercle. ${ }^{* *}$ specimen 2 reached saturation pressure of sensor 50

Figure 24: Effect of posterior capsular plication on peak subacromial pressure shown for each specimen. 50

Figure 25: Peak and average (mean \pm sd) subacromial contact pressure for no plication, 1 $\mathrm{cm}$ and $2 \mathrm{~cm}$ plication of the posterior capsule $(\mathrm{n}=8)$

Figure 26: Humerus axial rotation (deg) versus glenohumeral abduction angle (deg) for each specimen (pre-test data).

Figure 27: Plane of elevation (deg) versus glenohumeral abduction angle (deg) for each specimen (pre-test data). 
Figure 28: Humeral elevation ( $\mathrm{mm}$ ) versus glenohumeral abduction angle (deg) for each specimen (pre-test data).

Figure 29: Anterior translation ( $\mathrm{mm}$ ) versus glenohumeral abduction angle (deg) for each specimen (pre-test data). 55

Figure 30: Initially deltoid cable was to be redirected with eyelet attached to acromion. 57 Figure $31: 4.5 \mathrm{~mm}$ fracture fixation plates secured to the medial and lateral borders of the

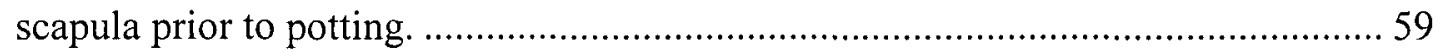

Figure 32: Greater tubercle comes into close proximity with acromion ..........................65 


\section{List of Tables}

Table 1: Loading distribution of deltoid and cuff muscles for previously published shoulder simulators.

Table 2: Constant rotator cuff muscle ratios.

Table 3: Calibration factors for calibration $\left(\mathrm{y}=\mathrm{Ax} \mathrm{x}^{\mathrm{b}}\right)$ of Tekscan sensors (model 5051).

* exceeded saturation pressure.

Table 4: Subacromial contact (peak and average pressure, force and area) averaged (mean \pm sd) for all specimens at the position of peak pressure throughout abduction cycle $(\mathrm{n}=8)$. 52

Table 5: Glenohumeral kinematics: abduction angle, plane of abduction, external rotation and anterior, superior humeral head translation averaged (mean \pm sd) for all specimens at the position of peak pressure throughout abduction cycle. ${ }^{*}$ denotes significant difference in external rotation for $1 \mathrm{~cm}$ plication group compared to no plication and a $2 \mathrm{~cm}$ plication $(\mathrm{p}<0.05)$. 


\section{List of Appendices}

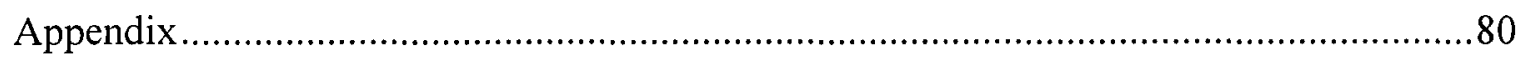

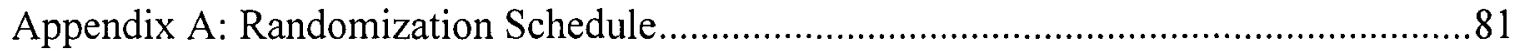

Appendix B: Peak Subacromial Pressure versus Abduction Angle...................................82

Appendix C.1: Shoulder Simulator - Engineering Drawings.........................................8

Appendix C.2: Polaris Passive Marker Tools - Engineering Drawings ...........................116 


\section{Glossary}

Abduction: Moving of a body part away from the central axis of the body. Shoulder abduction consists of elevation of the humerus away from the body in the frontal plane. Anterior: Anatomical direction in sagittal plane - situated toward the front of the body. Distal: Farther from a point of reference such as an origin, a point of attachment, or the midline of the body.

Flexion: Shoulder flexion consists of elevation of the humerus away from the body in the sagittal plane.

Frontal plane: A plane parallel to the long axis of the body and perpendicular to the sagittal plane that separates the body into front and back portions.

Lateral: Anatomical direction in frontal plane - situated away from the midline of the body.

Medial: Anatomical direction in frontal plane - situated near the midline of the body.

Plication: The operation of taking tucks in a structure to shorten it.

Posterior: Anatomical direction in sagittal plane - situated toward the back of the body.

Proximal: Nearer to a point of reference such as an origin, a point of attachment, or the midline of the body.

Sagittal plane: A plane parallel to the long axis of the body and perpendicular to the frontal plane that separates the body into left and right portions.

Scapular abduction: Elevation of the humerus away from the body in the scapular plane. 


\section{Chapter 1: Introduction}

Subacromial impingement (SI) syndrome is thought to produce pain by causing the greater tubercle to come into close proximity with the undersurface of the acromion, leading to compression of the rotator cuff. ${ }^{1}$ Acromial morphology is an accepted cause of subacromial impingement. Increasing curvature of the acromion may lead to impingement of the rotator cuff by decreasing the amount of subacromial space between the greater tubercle and the coracoacromial arch. ${ }^{2,3}$ In these cases, anterior acromioplasty may be indicated. ${ }^{4}$ The surgery can be performed open or arthroscopic and consists of removing the anterior undersurface of the acromion with a burr and debridement of the subacromial bursa. Results of anterior acromioplasty are inconsistent ${ }^{3}$ and failure to recognize secondary causes of SI may contribute to this variability.

Secondary causes of SI lead to compression of the rotator cuff as a result of another process. ${ }^{3}$ A number of secondary causes, independent of acromion morphology, are thought to contribute to the clinical syndrome. Secondary causes include shoulder instability $^{5}$, scapulothoracic weakness related to muscle inhibition ${ }^{6}$, neurological injury ${ }^{3}$, and posterior capsular tightness. ${ }^{7,8}$

The role of the posterior capsule in the development of SI is not well understood. Indirect evidence supports the hypothesis that a tight posterior capsule may lead to subacromial impingement. Ticker et al..$^{9}$ treated a group of nine patients with painful loss of internal rotation associated with refractory SI. Posterior capsular release resulted in substantial relief of pain. 
Harryman et al. ${ }^{7}$ in a cadaver study examined the effect of posterior capsular plication on humeral head translation during passive glenohumeral motion. Operatively tightening the posterior capsule resulted in increased superior and anterior translation during flexion which is hypothesized to increase subacromial contact pressure. However, Werner et al. ${ }^{10}$ found that plication of the posterior capsule had very little effect on glenohumeral translation during passive motion of the shoulder. Contrary to Harryman ${ }^{7}$, they reported a slight decrease in anterior translation during flexion. Lin et al. ${ }^{11}$ compared glenohumeral kinematics of patients with tight anterior and tight posterior shoulders during abduction in the scapular plane. They observed increased anterior and superior translation in patients with tight posterior shoulders but the authors did not compare groups with either controls or contralateral shoulders.

Posterior capsular tightness seems to be associated with SI but it is not clear whether it plays a causative role. Posterior capsule contraction may be an under recognized secondary cause of subacromial impingement leading to treatment failure. Definitive evidence of the relationship between posterior capsule plication and increased subacromial pressure would increase surgeon awareness and alter management in certain patients.

We hypothesize that a plicated posterior capsule leads to increased peak subacromial pressure during scapular abduction. The primary objective of this study was to evaluate the effect of posterior capsular plication on peak subacromial contact pressure during active abduction in the scapular plane using a cadaver shoulder simulator. The secondary objective was to compare glenohumeral kinematics at the position of peak pressure. 
Ten fresh-frozen human cadaver shoulder specimens were thawed, dissected and mounted on a custom designed shoulder simulator. With the scapula fixed, the deltoid and rotator cuff muscles were loaded in discrete static steps with a constant ratio to elevate the humerus in the scapular plane. For each treatment (no plication, $1 \mathrm{~cm}$ and 2 $\mathrm{cm}$ plication of the posterior capsule), pressure in the subacromial space and glenohumeral kinematics were recorded during abduction. The treatment order was randomly assigned to each specimen. Subacromial contact (peak pressure, average pressure, force and contact area) as well as glenohumeral kinematics at the position of peak pressure were compared using a repeated measures analysis of variance (ANOVA).

Summarizing the results of this thesis, peak subacromial pressures [mean \pm standard deviation (sd)] were similar between treatment groups: $345 \pm 152 \mathrm{kPa}, 410 \pm 213$ $\mathrm{kPa}$ and $330 \pm 164 \mathrm{kPa}$ for no plication, $1 \mathrm{~cm}$ and $2 \mathrm{~cm}$ plications, respectively ( $\mathrm{p}>0.05$ ). The glenohumeral abduction angle at the position of peak pressure was approximately 52 degrees which agrees with previous biomechanical studies. ${ }^{2,12,13}$ No significant differences in either glenohumeral abduction angle or plane of abduction at the position of peak pressure were noted between treatment groups. A statistically significant difference was observed in axial rotation of the humerus following a $1 \mathrm{~cm}$ plication compared to either no plication or a $2 \mathrm{~cm}$ plication although the clinical significance of two degrees is questionable. No significant differences were found for either superior or anterior translation of the humeral head at the peak pressure position.

The main strength of the current study is the ability to isolate the effect of posterior capsular plication on subacromial pressure and glenohumeral kinematics. Results show that posterior capsular plication in isolation does not increase peak 
subacromial pressure during abduction in the scapular plane nor does it significantly alter glenohumeral kinematics at this position. It is generally believed that a tight posterior shoulder with positive impingement signs originates from a thick and shortened capsule but a recent case report suggests that it may also originate from muscular contractures of the infraspinatus and teres minor. ${ }^{14}$ Our findings indirectly support this hypothesis.

The posterior capsule is most stretched at the limits of flexion where capsular tightness may contribute to obligate anterior and superior translation and increased subacromial pressure as suggested by Harryman et al. $^{7}$ A simulator for active shoulder flexion accompanied with internal rotation must be developed to fully understand the role of posterior capsular tightness as a factor in causation of SI. 


\section{Chapter 2: The Shoulder}

An appropriate model to study the relationship between posterior capsule plication and subacromial pressure is the fresh frozen cadaver shoulder because the effect of capsular plication in isolation may be studied. This chapter reviews shoulder biomechanics and previously published shoulder simulators.

\subsection{Anatomy and Biomechanics of the Glenohumeral Joint}

The large range of motion in the shoulder joint is due to the synergy between three distinct joints: the glenohumeral $(\mathrm{GH})$, acromioclavicular $(\mathrm{AC})$ and sternoclavicular (SC) joints, and two sliding spaces: the subacromial and scapulothoracic spaces (Figure 1). Through the complex interaction of all three joints, the shoulder provides the largest range of motion of any joint in the human body. ${ }^{15}$

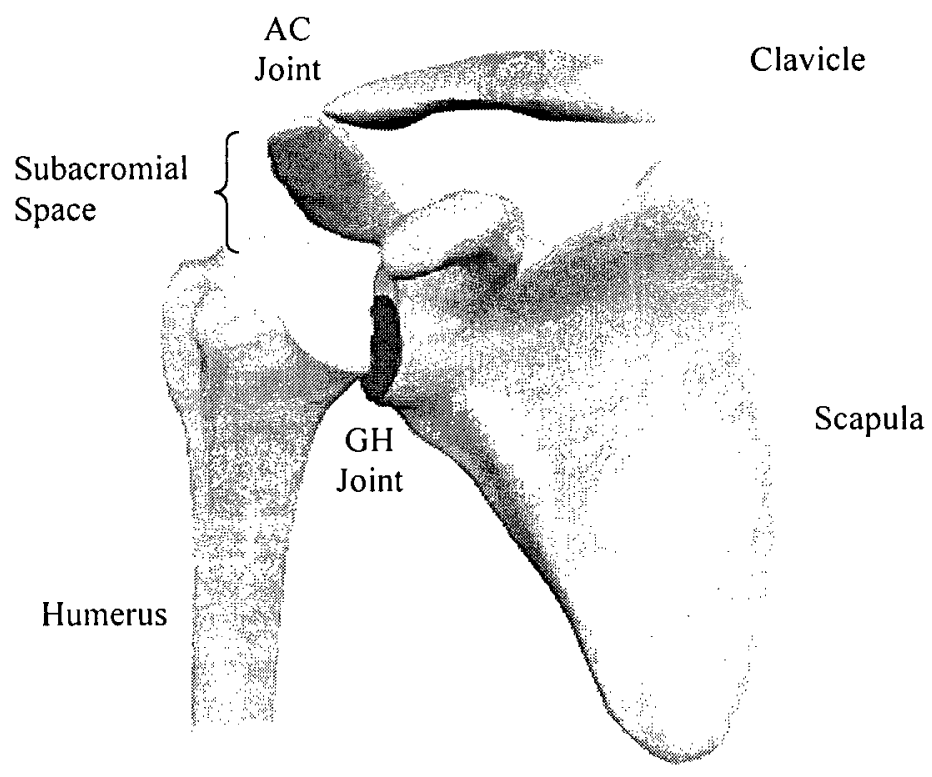

Figure 1: The shoulder is composed of the glenohumeral (GH), acromioclavicular ( $\mathrm{AC}$ ) and sternoclavicular joints. ${ }^{16}$ 
Our focus is directed to the glenohumeral joint and subacromial space. The GH joint is often described as a ball and socket joint similar to the hip joint but in reality, the large humeral head can rotate and slide on the glenoid. The humeral head is spherical and its radius of curvature is much larger than that of the glenoid. The radius of curvature of the humeral head ranges from $20-30 \mathrm{~mm}$ and is smaller in women than men. ${ }^{17}$

The center of the humeral head is translated medially from the long axis of the humerus and is offset posteriorly (Figure 2). The medial and lateral offsets range from 4 to $14 \mathrm{~mm}$ and -2 to $10 \mathrm{~mm}$ respectively. ${ }^{17}$
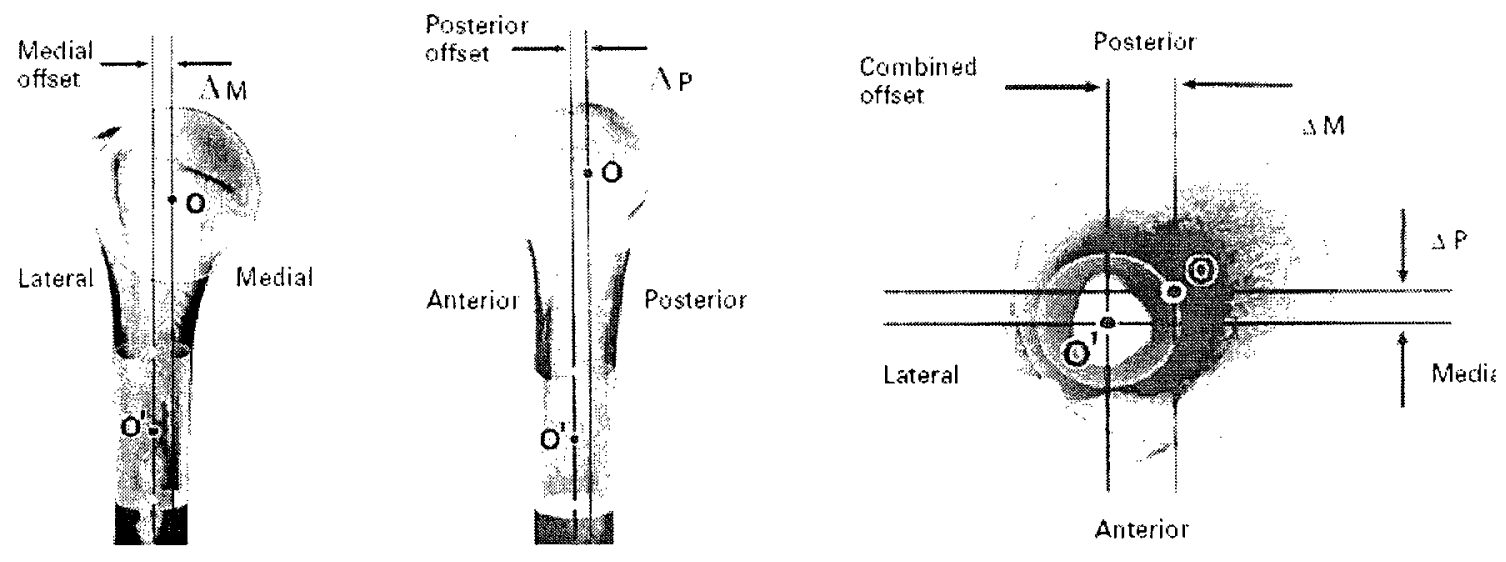

Figure 2: Medial and Posterior Offset of Center of Humeral Head. ${ }^{18}$

$O$ - center of humeral head; $O^{\prime}$ - center of humeral canal.

A lateral view of the glenoid (Figure 3a) shows the pear-shaped profile of the glenoid: the mean vertical dimension is $35 \mathrm{~mm}$ and the mean transverse diameter is 25 $\mathrm{mm} .{ }^{19}$ The frontal view reveals that the glenoid has a medial vertical tilt of approximately 5 degrees (Figure 3b). A large range in version (angle formed by the plane of the glenoid with respect to the plane of the scapula) of the glenoid can be found: according to one study, $75 \%$ of shoulders are retroverted with an average of 7.4 degrees (Figure $4 b$ ) and 
$25 \%$ are anteverted from 2 to 10 degrees. $^{19}$ It is suggested that the retroversion is important for maintaining clinical stability and counteracting anterior translation of the humeral head.

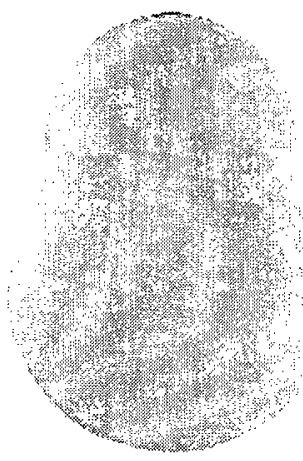

(a)

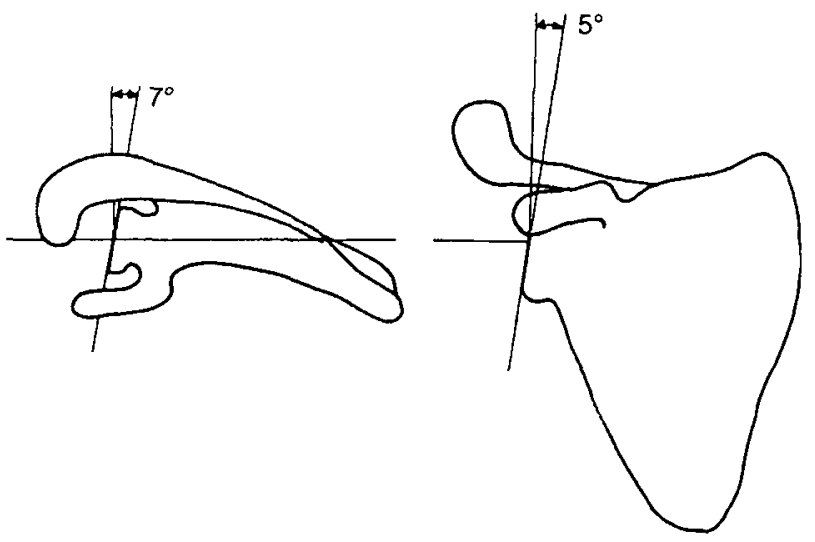

(b)

Figure 3: (a) pear-shaped profile of glenoid (lateral view) ${ }^{19}$ and (b) retroversion and vertical tilt of glenoid. ${ }^{20}$

The osseous mismatch in radius of curvature between the humerus and glenoid is between $8-9 \mathrm{~mm}$ but when one considers the cartilage and glenoid labrum, the mismatch is approximately $0.1 \mathrm{~mm} .^{21}$ The glenohumeral mismatch allows for the gliding of the humeral head against the glenoid providing large range of motion. During elevation, the center of the humeral head translates on average $1.1 \mathrm{~mm}$ superiorly and $1.5 \mathrm{~mm}$ in the anterior-posterior direction. $^{22}$

The subacromial space is the space formed between the humeral head and the coracoacromial arch which consists of the undersurface of the acromion, the coracoacromial ligament and corocoid process (Figure 4). As the humerus is abducted, the humeral head translates superiorly and comes into contact with the acromion. The 
pressure exerted on the supraspinatus tendon by the roof of the coracoacromial arch is termed impingement.

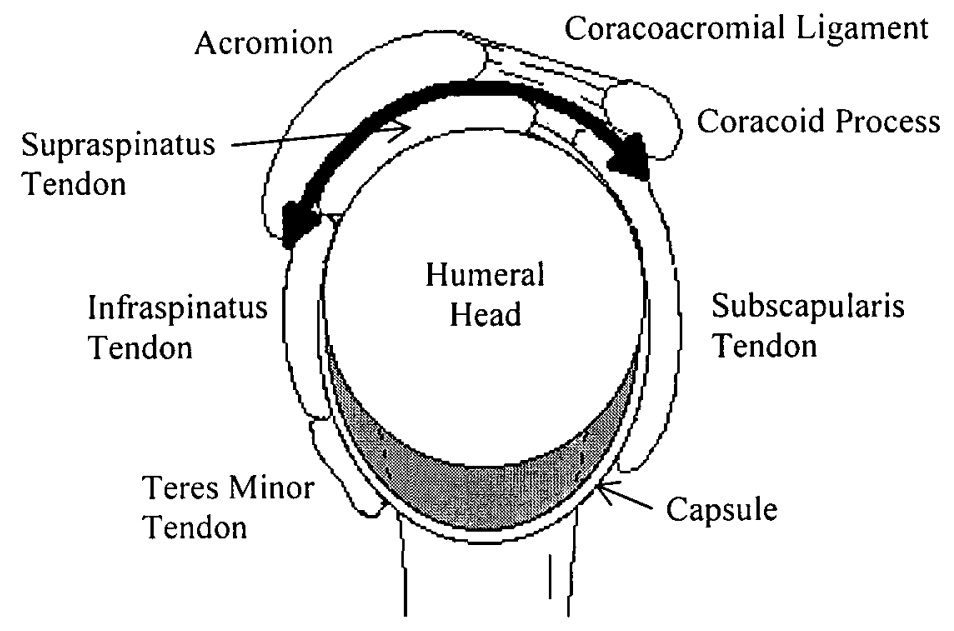

Figure 4: Lateral cross-section of glenohumeral joint showing coracoacomial arch (arrow) which spans from the posterior aspect of the acromion to the coracoid process. ${ }^{23}$

The clinical stability of the glenohumeral joint is provided by static (negative intra-articular pressure, joint capsule, labrum and ligaments) and dynamic (rotator cuff muscles) stabilizers. The joint capsules attach laterally to the proximal humerus around the edge of the articular cartilage and attach medially to the glenoid rim and provide passive constraints.

The labrum serves to absorb shear forces and distribute stresses over a larger area on the glenoid reducing peak contact stresses. It has also been suggested that the labrum provides increased clinical stability of the humeral head on the glenoid, assists with lubrication and serves as an attachment for the glenohumeral ligament. ${ }^{24}$

Ligaments at the glenohumeral joint consist of the capsular ligaments (superior, mid and inferior) attached to the anterior capsule, the glenohumeral ligament, the 
coracohumeral ligament and the coracoacromial ligament (Figure 5). The coracoacromial ligament spans from the acromion to the coracoid process forming the coracoacromial arch. The coracohumeral ligament spans from the coracoid process to the anatomical neck of the humerus near the greater tubercle. The capsular ligaments strengthen the anterior capsule and contribute to clinical glenohumeral stability. The superior glenohumeral ligament and coracohumeral ligaments aid in the prevention of downward displacement of the humeral head. ${ }^{24}$

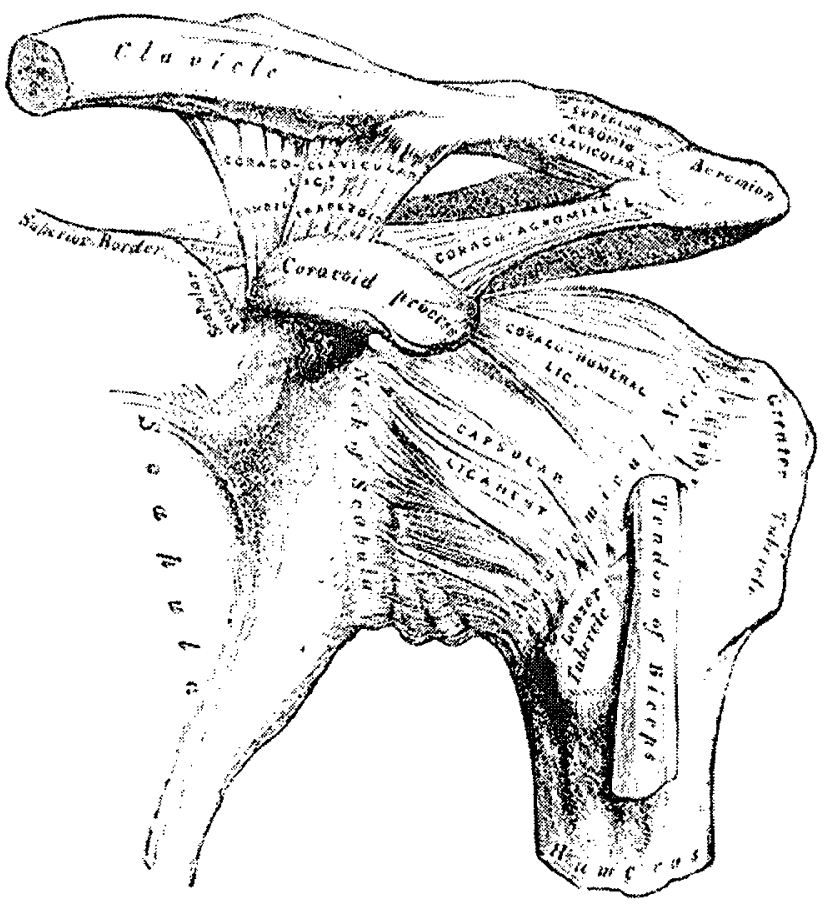

Figure 5: Liga ments at the glenohumeral joint: glenohumeral ligaments (capsular ligament), coracoacromial ligament and coracohumeral ligament. ${ }^{25}$

The rotator cuff acts to stabilize the joint, provide great freedom of movement and fixes the fulcrum of the upper extremity against which the deltoid can contract to elevate the humerus. The rotator cuff consists of four muscles that act around the GH joint: the 
supraspinatus (SSP), infraspinatus (ISP), teres minor (TM) and subscapularis (SUB) (Figure 6).

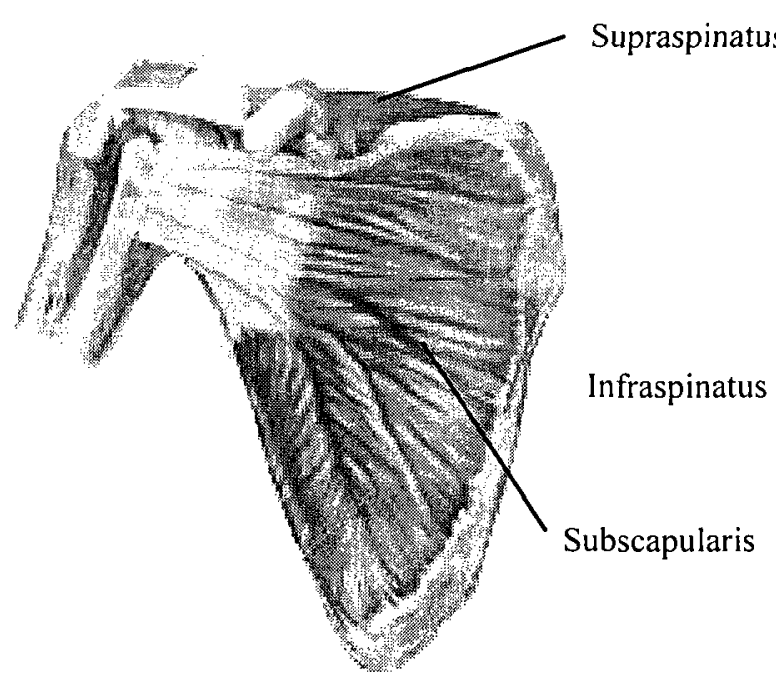

Anterior View

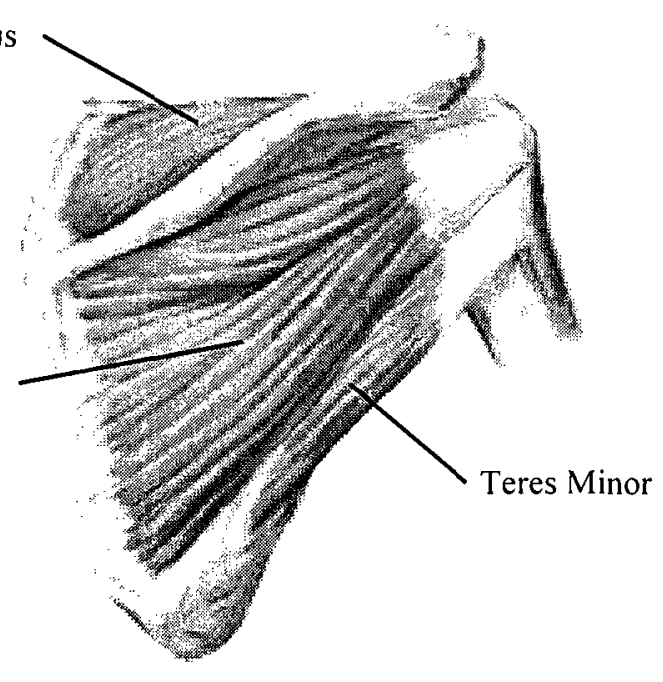

Posterior View

Figure 6: Rotator cuff (SSP, ISP, TM and SUB) and deltoid act to provide great range of motion and stabilize the glenohumeral joint. ${ }^{26}$

The supraspinatus assists with elevation and also acts as a humeral head depressor. The infraspinatus (ISP) and teres minor (TM) are both posterior to the scapula and act to externally rotate the humerus; the subscapularis (SUB) is anterior to the scapula and thus acts to internally rotate the arm. The ISP, TM and SUB also act as humeral head depressors.

The deltoid muscle is the primary abductor force and can be separated in three separate heads: anterior, middle and posterior (Figure 7). The anterior deltoid muscle originates on the anterior border of the clavicle, the middle deltoid on the superior surface of the acromion and the posterior deltoid on the inferior margin of the scapula spine. All 
three heads insert on the humeral tuberosity. The middle deltoid acts mainly to elevate the humerus in the scapular plane, the anterior deltoid assists with forward flexion while the posterior deltoid assists with extension.

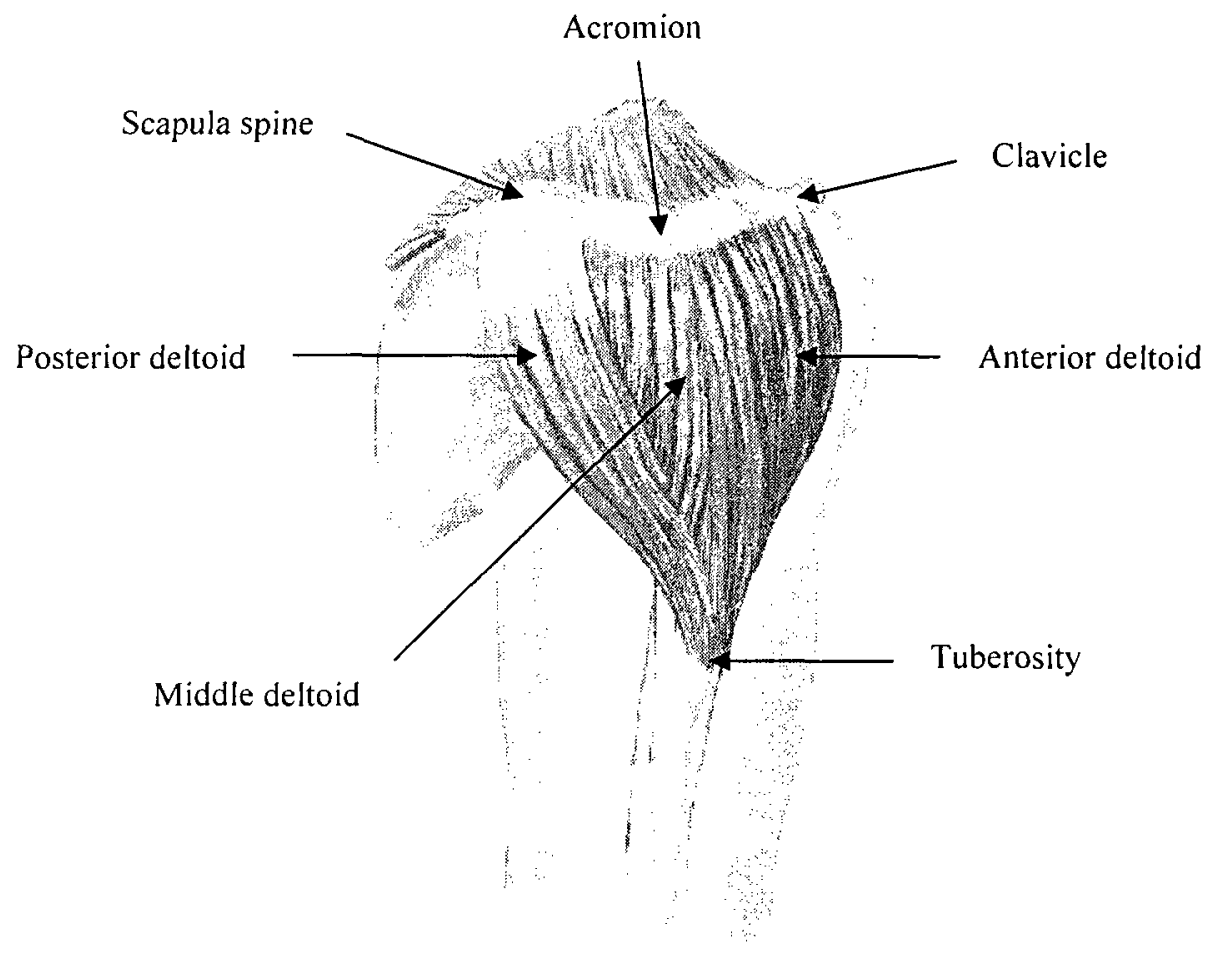

Figure 7: Anterior, middle and posterior deltoids. ${ }^{27}$ 


\subsection{Shoulder Kinematics}

To describe the motion of the shoulder, three anatomical planes are used: the sagittal, coronal and scapular planes. The scapular plane is rotated externally from the coronal plane by approximately 30 degrees. ${ }^{28}$

Elevation of the humerus in the sagittal plane is termed forward flexion or elevation, elevation in the coronal plane is abduction and elevation in the scapular plane is termed scapular abduction. Figure 8 illustrates forward flexion (A), abduction (C) and internal and external rotation (D). The average forward elevation in men is 167 degrees and in women is 171 degrees. ${ }^{20}$ Elevation in the plane of the scapula is considered more functional because the capsule is not twisted and the musculature of the shoulder is optimally aligned for elevation. ${ }^{20}$
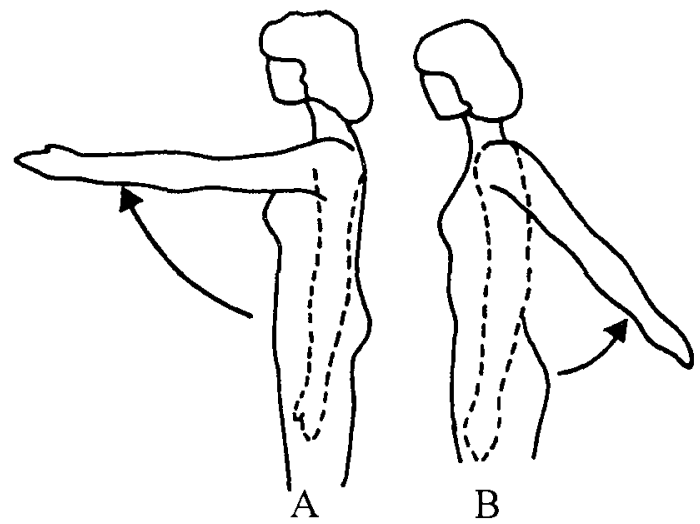

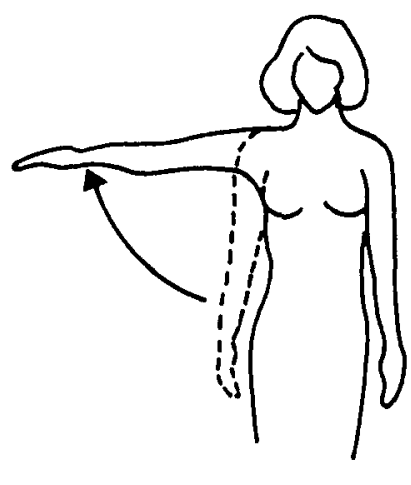

$\mathrm{C}$

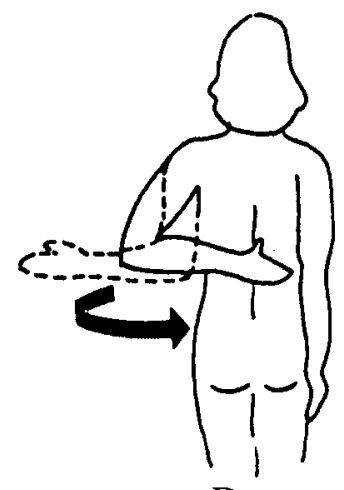

$\mathrm{D}$

Figure 8: Forward flexion (A) and extension (B), abduction (C) and external and internal rotation (D). ${ }^{20}$ 
When describing the abduction or elevation angle one needs to properly define the reference frame. In this paper, shoulder abduction will describe the elevation angle of the humerus with respect to the sagittal plane and glenohumeral abduction will describe the elevation angle of the humerus with respect to the fixed scapula. As the humerus is elevated, the scapula also undergoes an upward rotation (Figure 9). The resulting ratio of shoulder abduction to glenohumeral abduction is approximately $1.5: 1 .{ }^{29}$
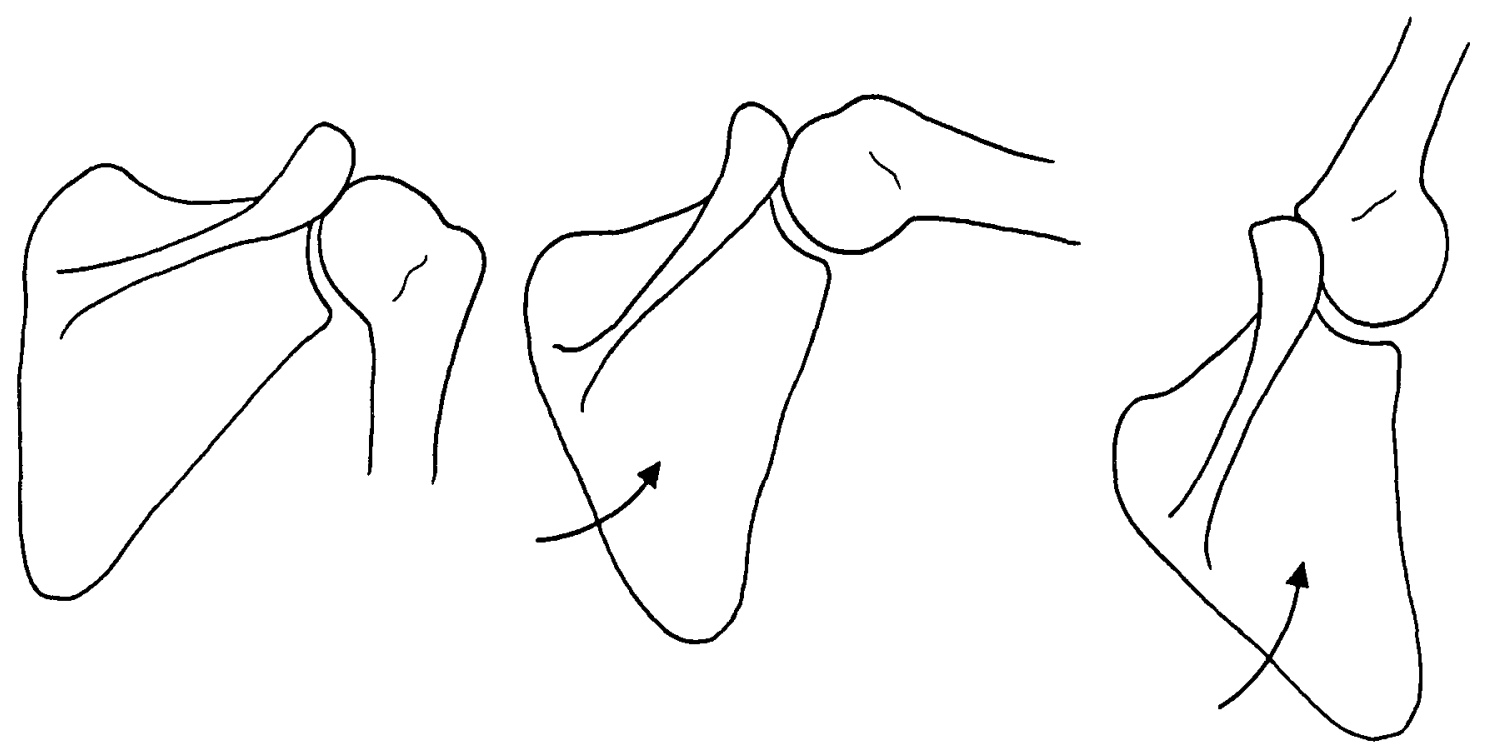

Figure 9: Upward rotation of scapula during shoulder elevation. ${ }^{20}$ 


\subsection{In-vitro Shoulder Simulators}

Wuelker et al. have designed a dynamic shoulder simulator to study the subacromial pressure in human cadavers for different positions of abduction in the scapular plane. ${ }^{12,30-32}$ With the scapula fixed, the simulator allows for the application of forces with hydraulic cylinders along the lines of action of the deltoid and rotator cuff muscles to elevate the arm in the scapular plane. The anterior, middle and posterior deltoid as well as the supraspinatus, subscapularis and infraspinatus combined with teres minor were loaded in the simulation. The ratios of forces were based on the crosssectional area of each muscle and remained constant for all angles of abduction (Table 1, p.16). Subacromial contact pressure was measured in 10 cadaver specimens with the Emed capacitive pressure sensor array (Novel, Munich Germany). Pressure was highest between 51 and 82 degrees of glenohumeral abduction in the scapular plane ( 68 degrees on average). ${ }^{31}$

Payne et al. used a dynamic model to evaluate the dynamic and static contributions of each muscle to subacromial impingement. ${ }^{2}$ Their dynamic model was similar to Wuelker with differences including the loading regimen and the inclusion of the biceps muscle. The loading regimen was determined based on EMG data as a function of glenohumeral abduction and the cross-sectional area of each muscle. Muscle ratios at 90 degrees of glenohumeral abduction are included in Table 1 (p.16). A constant load of $10 \mathrm{~N}$ was applied to the long head of the biceps to simulate the weak head depressing effect of the biceps tendon. Payne et al. measured pressure in five specific locations (anterolateral, posterolateral, anteromedial, CA ligament and coracoid) with miniature pressure transducers in each location. The highest pressures were recorded at 
the anterolateral acromion. They determined that anterior pressures decreased by $99 \%$ after anterior acromioplasty in cadavers with type III acromia. ${ }^{2}$

To achieve abduction in the scapular plane, Payne et al. ${ }^{2}$ loaded the middle deltoid with the rotator cuff muscles balanced. In addition, the anterior and/or posterior deltoid required minimal loading in a small number of specimens to re-align the motion in the scapular plane. The authors defined criteria to determine when loading of the anterior or posterior deltoid was necessary: movement of the arm greater than 30 degrees anteriorly or posteriorly from true scapular abduction required additional muscle loading to balance and stabilize the arm. Wuelker et al. ${ }^{12}$ loaded the middle deltoid and accepted spontaneous deviations from the scapular plane to keep the arm undisturbed from external forces.

Various authors have published similar cadaver shoulder simulators to investigate conditions other than shoulder impingement. Bono et $\mathrm{al}^{33}$ designed a static shoulder simulator to study the effect of displacement of greater tubercle fractures on shoulder mechanics. They used static weights to apply loads to the cuff muscles and a servo hydraulic actuator to transmit load to the deltoid. Mura et al. ${ }^{34}$ also designed a dynamic shoulder model to study the effect of infraspinatus disruption on glenohumeral torque and superior migration. They used static weights to apply loads to all muscles groups. In both models, the same muscle groups were loaded: deltoid, supraspinatus, infraspinatus with teres minor and subscapularis. The loading distributions of the muscles for these two models are also shown in Table 1. 
Table 1: Loading distribution of deltoid and cuff muscles for previously published shoulder simulators.

\begin{tabular}{cccccc}
\hline & Wuelker & Payne & Bono & Mura & $\begin{array}{c}\text { Mean } \\
\text { (SD) }\end{array}$ \\
\hline Deltoid & 43 & 60 & 55 & 44 & $50(8)$ \\
SSP & 9 & 5 & 9 & 12 & $9(3)$ \\
ISP+TM & 22 & 23 & 15 & 24 & $21(4)$ \\
SUB & 26 & 12 & 21 & 22 & $20(6)$ \\
\hline
\end{tabular}




\section{Chapter 3: Shoulder Impingement}

\subsection{Definition, Diagnosis and Treatment}

Neer was the first to describe subacromial impingement (SI) syndrome in $1972 .^{4}$ SI is thought to produce pain by causing the greater tubercle to come into close proximity with the undersurface of the acromion, leading to compression of the rotator cuff. ${ }^{1}$ Impingement of the supraspinatus tendon has been demonstrated to occur primarily at the acromial end of the coracoacromial ligament and anterior tip of the acromion during arcs of flexion and internal rotation. ${ }^{35}$

Clinical diagnosis of shoulder impingement consists of reproducing the pain by recreating the condition of impingement of the rotator cuff between the greater tubercle and the coracoacromial arch. The Neer test is carried out with passive forward elevation of the arm in neutral rotation while holding the scapular fixed. ${ }^{37}$ The Hawkins test is carried out with glenohumeral internal rotation from a starting position of 90 degrees of forward elevation and 90 degrees of elbow flexion with neutral rotation. ${ }^{1}$ In both cases, a positive test requires reproduction of pain. Three dimensional MRI analysis has shown a significant position-dependant decrease in the acromiohumeral distance in patients with impingement syndrome compared with healthy controls. ${ }^{38}$

According to Bigliani and Levine ${ }^{3}$, the causes of SI can be broadly classified into intrinsic (intratendinous) and extrinsic (extratendinous) factors. Intrinsic factors include rotator cuff weakness, shoulder overuse and degenerative tendinopathy. Extrinsic etiologies can further be classified into primary and secondary. 
Primary causes of SI lead to direct compression between the rotator cuff and the acromion. Acromial morphology is a thoroughly studied cause of primary, extrinsic subacromial impingement. The acromial morphology has been classified into three shapes; flat (type I), curved (type II) and hooked (type III) (Figure 10). ${ }^{3}$ Increasing curvature of the acromion may lead to impingement of the rotator cuff by decreasing the amount of subacromial space between the greater tubercle and the coracoacromial arch.
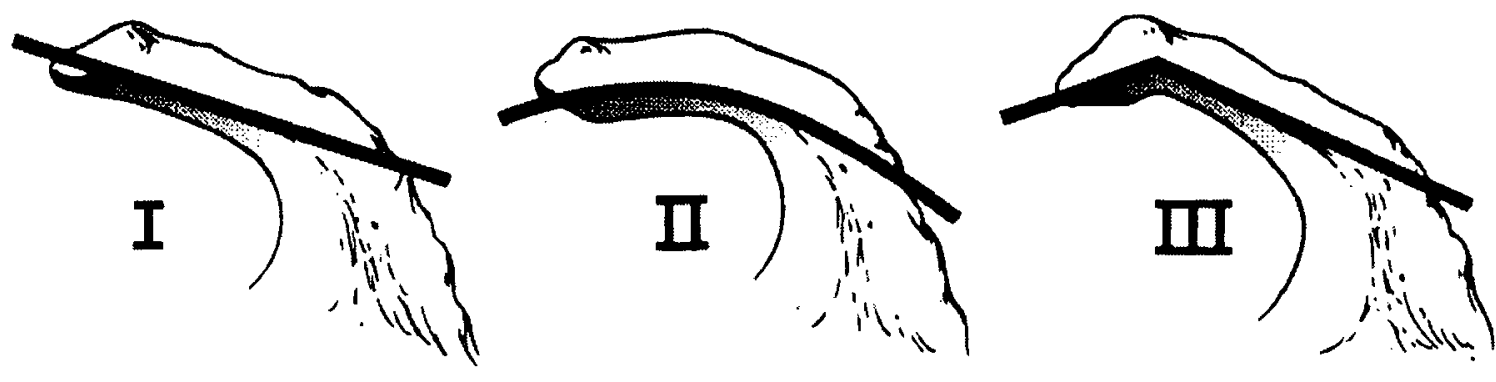

Figure 10: Acromion Shapes. ${ }^{36}$

Secondary causes of SI lead to compression of the rotator cuff as a result of another process. ${ }^{3}$ A number of secondary factors, independent of acromion morphology, are thought to contribute to the clinical syndrome. Secondary causes include shoulder instability $^{5}$, scapulothoracic weakness related to muscle inhibition ${ }^{6}$, neurological injury ${ }^{3}$ and posterior capsular tightness. ${ }^{7}$

Non-operative treatment remains the most common intervention and includes modification of activity, use of non-steroidal anti-inflammatory (NSAID's) medications, subacromial injections of steroids and physical therapy. ${ }^{3}$ The duration of non-operative treatment ranges from 12 to 18 months. ${ }^{3}$ Satisfactory results are achieved in $67 \%$ of cases according to a study by Morrison et al. ${ }^{39}$ 
If non-operative treatments fail, anterior acromioplasty may be indicated. ${ }^{4}$ The technique can be performed with open or arthroscopic surgery and consists of removing the anterior undersuface of the acromion with a burr and debridement of the subacromial bursa. Satisfactory results of open anterior and arthroscopic subacromial acromioplasty range from $46-97 \% .^{3}$ Failure to recognize secondary causes of subacromial impingement syndrome may contribute to this variability. Soyer et al. ${ }^{40}$ examined the correlation between the degree of acromial resection and functional outcomes following arthroscopic subacromial decompression. The measurement of the anterior acromial protuberance preoperatively was statistically less than the amount postoperatively. However, no correlation was found between the degree of resection and functional outcome as determined by the Constant score. The multifactorial nature of impingement syndrome is reinforced by the results of this study as technical success did not correlate with functional outcomes. 


\subsection{Impingement and Subacromial Pressure}

Subacromial contact pressure has been previously used as an outcome measure of impingement syndrome. Nordt et al. ${ }^{41}$ conducted a clinical study in which subacromial contact pressure was measured intra-operatively with a balloon catheter prior to and following subacromial decompression. Pressures increased to 90 degrees of clinical abduction in all cases to a mean pressure of $35.6 \mathrm{mmHg}$ (range 2.0 to $112.0 \mathrm{mmHg}$ ). Pressure was always higher with the shoulder in external rotation than in internal rotation at $90^{\circ}$ of clinical abduction. Pressures continued to increase at 180 degrees of hyperabduction to a mean pressure of $50.1 \mathrm{mmHg}$ (range 1.2 to 112.7). The authors concluded that contact pressure decreased significantly in all positions following decompression.

Werner et al. ${ }^{13}$ measured subacromial pressures in vivo in 11 healthy patients. An arterial catheter ( $1 \mathrm{~mm}$ diameter) was inserted into the subacromial bursa. Pressure was measured at $0,60,90$ degrees and maximum abduction in the scapular plane and during maximum external and internal rotation with the arm at the side. Pressure peaked at 90 degrees of clinical abduction ( $66.9 \mathrm{~mm} \mathrm{Hg}$, range 8 to $231 \mathrm{~mm} \mathrm{Hg}$ ) and was highest in internal rotation as compared to external rotation with the arm at the side. The authors did not investigate the effect of rotation at different abduction angles. They also noted that patients with strong external rotation with arm at their side had lower subacromial pressures.

Wuelker et al. $^{31}$ measured subacromial pressures during simulated active abduction in the scapular plane in 10 fresh-frozen cadaver shoulders. Peak pressure 
averaged $566 \mathrm{kPa}$ (range 174 to $1280 \mathrm{kPa}$ ) and occurred at a mean glenohumeral abduction angle of 68 degrees (range 51 to 82 degrees). Removing the supraspinatus had little effect on the subacromial pressure $(8 \%$ decrease $)$ while removing the infraspinatus/teres minor and subscapularis led to a significant increase in pressure $61 \%$, $\mathrm{p}<0.05)$

Similarly, Payne et al. ${ }^{2}$ measured subacromial pressure in a cadaver shoulder model simulating active abduction in the scapular plane. They found that the peak pressure was under the anterolateral aspect of the acromion and that it occurred at $48^{\circ}$ of glenohumeral abduction. The mean peak pressure was highest for a type III acromion $(1186 \pm 1675 \mathrm{kPa})$ as compared to a type I or II $(52 \pm 52 \mathrm{kPa})$ acromion. Failure to simulate a supraspinatus force decreased acromial pressure by $52 \%$ in shoulders with type III acromion. Finally, they found that pressures decrease by $99 \%$ following an anterior acromioplasty.

In summary, subacromial pressure has been previously recorded as a measure of shoulder impingement syndrome. In-vitro studies ${ }^{2,31}$ have shown that subacromial pressure can be affected by acromial shape ${ }^{2}$ or rotator cuff dysfunction. ${ }^{2,31}$ 


\subsection{Impingement and Kinematics}

In the normal shoulder, superior translation of the humeral head with active arm elevation is thought to be minimal due to the depressor effect of the rotator cuff. ${ }^{42}$ In symptomatic patients, altered glenohumeral kinematics during arm elevation, particularly superior or anterior humeral head translation, inadequate external rotation and decreased scapular upward rotation is believed to decrease subacromial space and increase pressure. $^{43}$

Patients with impingement syndrome have been shown to have altered glenohumeral kinematics. Deutsch et al. ${ }^{44}$ measured superior translation of the humeral head with planar x-ray in normal patients and patients with impingement and rotator cuff tears. They found significant superior translation of the humeral head during abduction in the scapular plane in patients with impingement and in patients with rotator cuff tears as compared to healthy controls. They hypothesized that this was a dynamic phenomenon from rotator cuff dysfunction. No difference between patients with impingement and rotator cuff tears was found. They did not investigate other potential contributing factors to humeral head translation such as posterior capsular tightness.

Ludewig et $\mathrm{al}^{8}{ }^{8}$ measured humeral head translation during active abduction in the scapular plane using an electromagnetic tracking system (FASTRAK, Polhemus Inc.). Patients with impingement syndrome had significantly greater anterior translation during active abduction but no difference in terms of superior translation was noted between both groups. A correlation between increased anterior translation and loss of cross-body adduction was found during elevation between 90-120 degrees. The authors conclude that 
further studies are needed to examine the relationship between posterior capsule tightness, rotator cuff function and abnormal humeral translations.

Hallström et al. ${ }^{43}$ compared glenohumeral kinematics of controls and patients with impingement during active abduction using dynamic radiostereometric analysis. Both groups exhibited similar patterns of flexion and rotation during active abduction. No difference in peak abduction angle was noted between both groups. During abduction in the scapular plane, the center of the humeral head translated in the medial, superior and anterior directions. In the impingement group, the center of the humeral head translated slightly more $(1-1.5 \mathrm{~mm})$ superiorly while no difference was found in either medial or anterior displacement. In normal patients, superior translation increased to 20 degrees of glenohumeral abduction to a maximum of approximately $1.3 \mathrm{~mm}$.

In summary, abnormal kinematics can lead to a decrease in subacromial space and increase in pressure between the rotator cuff and the undersuface of the acromion. Studies have demonstrated increased superior or anterior translation of the humeral head in patients with impingement syndrome $e^{8,43,44}$ which seems to indicate that another process other than acromial morphology may be responsible for SI. 


\subsection{Role of Posterior Capsule in Shoulder Impingement}

The role of the posterior capsule in the development of SI is not well understood. Clinically, posterior capsule tightness is felt to be manifested by limitations in internal rotation and cross-body adduction. ${ }^{7,45,46}$ Tyler et al ${ }^{46}$ demonstrated that a $1 \mathrm{~cm}$ loss of cross-body adduction results in a corresponding loss of 4 degrees of internal rotation in patients with impingement syndrome. They suggest that the loss of cross-body adduction is caused by a tight posterior capsule and thus provide indirect evidence that posterior capsule tightness is manifested clinically in patients with impingement syndrome. Ticker et al. ${ }^{9}$ performed a posterior capsular release in nine patients with painful loss of internal rotation associated with refractory SI which resulted in substantial relief of pain.

In a cadaver study, Gerber studied the passive range of motion of the glenohumeral joint following selective capsulorrhaphy. ${ }^{47}$ They observed a significant loss of internal rotation following a $1 \mathrm{~cm}$ plication of the posterior capsule. Harryman et al. ${ }^{7}$ examined the effect of posterior capsular plication on humeral head translation during passive glenohumeral motion in a cadaver study. Tightening the posterior capsule resulted in increased superior and anterior translation during flexion. The observed 'obligate' translation at the extreme of flexion has been suggested to decrease subacromial space and increase pressure leading to SI. However, Werner et al. ${ }^{10}$ found that plication of the posterior capsule had very little effect on glenohumeral translation during passive motion of the shoulder.

Lin et al. ${ }^{11}$ compared glenohumeral kinematics of patients with tight anterior and tight posterior shoulders during abduction in the scapular plane. They observed increased 
anterior and superior translation in patients with tight posterior shoulders but the authors did not compare groups with either controls or contralateral shoulders.

Posterior capsular tightness seems to be associated with SI but it is not clear whether it plays a causative role. Younger patients with SI and those without significant acromial abnormalities have had inconsistent results from anterior acromioplasty. Posterior capsule contraction may be an under recognized secondary cause of subacromial impingement leading to treatment failure. Definitive evidence of the relationship between posterior capsule tightness and increased subacromial pressure would increase surgeon awareness and alter management in certain patients. Specifically, intensive posterior capsule stretching would be indicated and if unsuccessful, posterior capsule release. ${ }^{15}$ This would result in earlier return of shoulder function, fewer inappropriate surgical interventions, and reduced health care costs. 


\section{Chapter 4: Research Methods}

\subsection{Design of Shoulder Simulator}

The simulator was designed to simulate shoulder abduction in the scapular plane and was adapted from previously published cadaveric shoulder simulators. ${ }^{2,32-34}$ Abduction in the scapular plane was first examined in this study because all muscle forces are aligned in this plane during this motion. In future research, a flexion simulator will be developed based on this first generation simulator.

The shoulder simulator (Figure 11 and 12) was secured to a materials testing load frame (MTS Sintech 1/G, MTS Systems Corp, Eden Prairie, MN). The primary abductor force (DEL) was generated with the MTS load frame and the load was held at discrete static positions while the subacromial contact pressure (section 4.2) and glenohumeral kinematics (section 4.3) were measured. A 5 kN load cell (100-090-859, MTS Systems Corp, Eden Prairie, MN) with a non-linearity of $0.04 \%$ and hysteresis of $0.03 \%$ measured the deltoid force. For each static position, the rotator cuff forces were applied with static weights: supraspinatus (SSP), infraspinatus combined with teres minor (ISP+TM) and subscapularis (SUB). A weight was attached distally to the humerus (A) to simulate the effective arm weight with the elbow fully extended. Passive marker tools attached on the humerus (B) and the scapula (C) were used for glenohumeral tracking. 


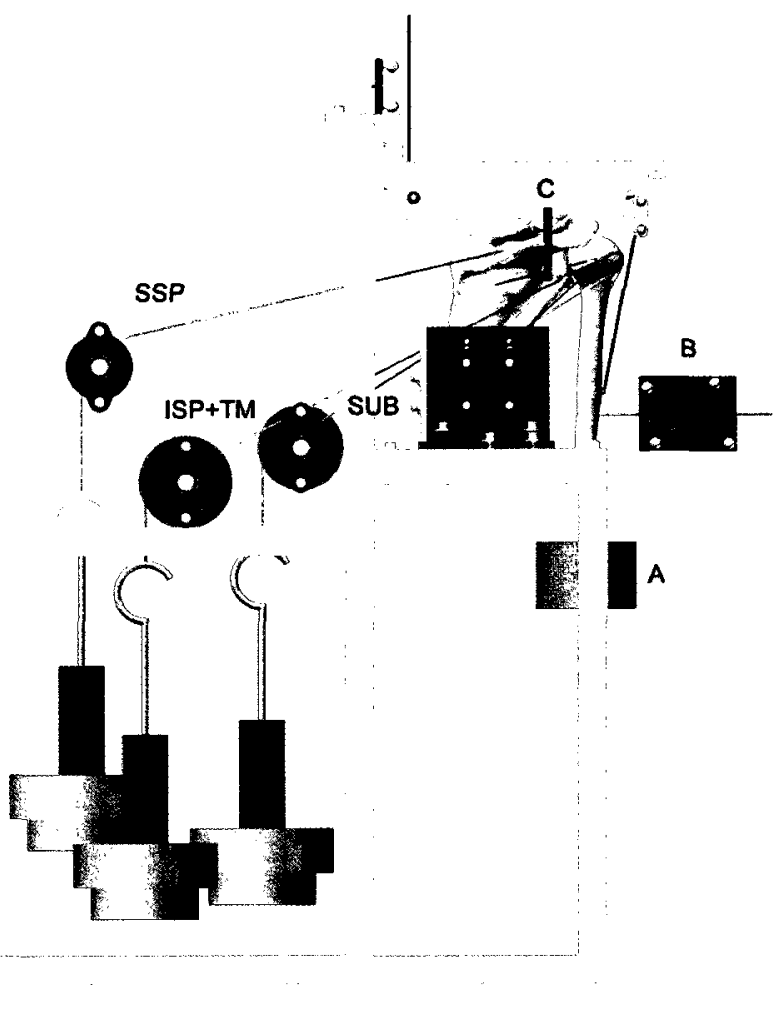

Figure 11: Schematic Representation of shoulder simulator.

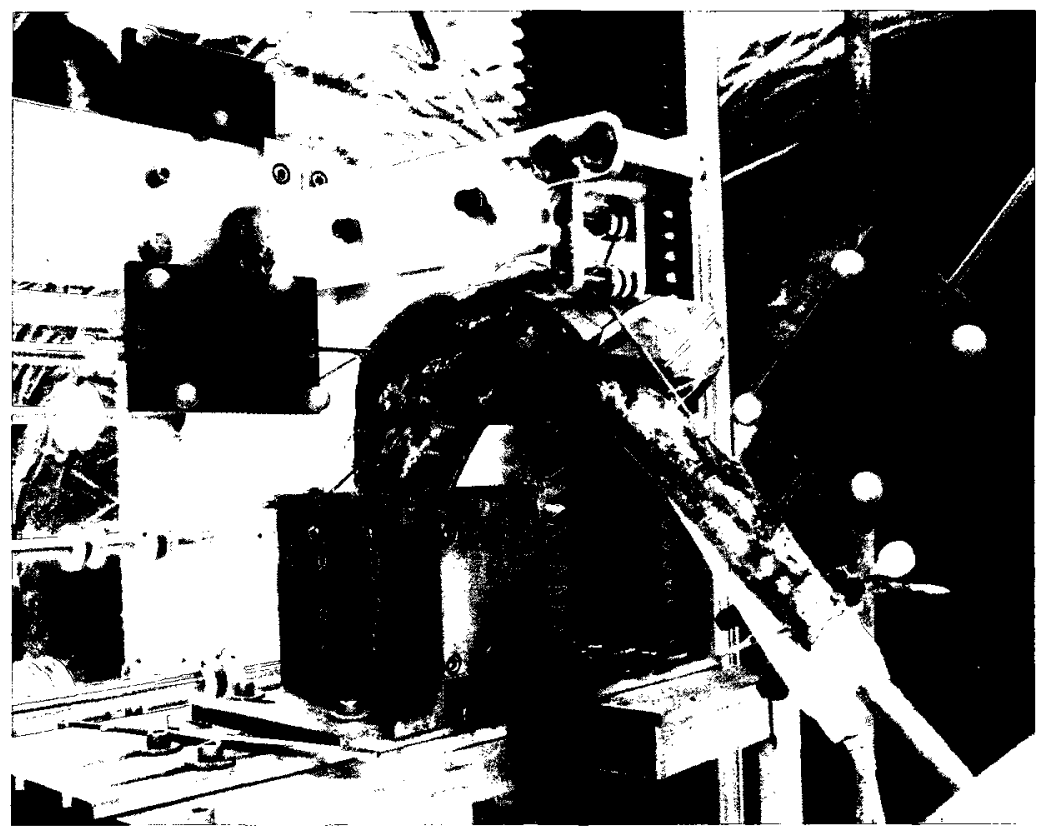

Figure 12: Shoulder Simulator. 
The reference position for the design of the simulator is the center of the humeral head. A solid model of the humerus and scapula were downloaded from the Université Libre de Bruxelles (ULB) 2009 at http://homepages.ulb.ac.be/ anatemb/welcome.html and imported into Autodesk Inventor R7 (Autodesk Inc., San Rafael, CA) to facilitate the design of the simulator. Engineering drawings of the shoulder simulator can be found in Appendix C.1.

The bottom one third of the scapula is fixed with bismuth in a potting cup such that the plane of the scapula (see definition in section 4.3) is parallel to the simulator and the medial border is vertical.

The potting cup is fixed in the simulator by aligning the center of the humeral head with the alignment fixture (Figure 13). The superior-inferior height (SI) of the potting cup is adjusted with a series of holes securing the potting cup to the X-Y table. The antero-posterior (AP) and medio-lateral (ML) adjustments are achieved with the X-Y table. 


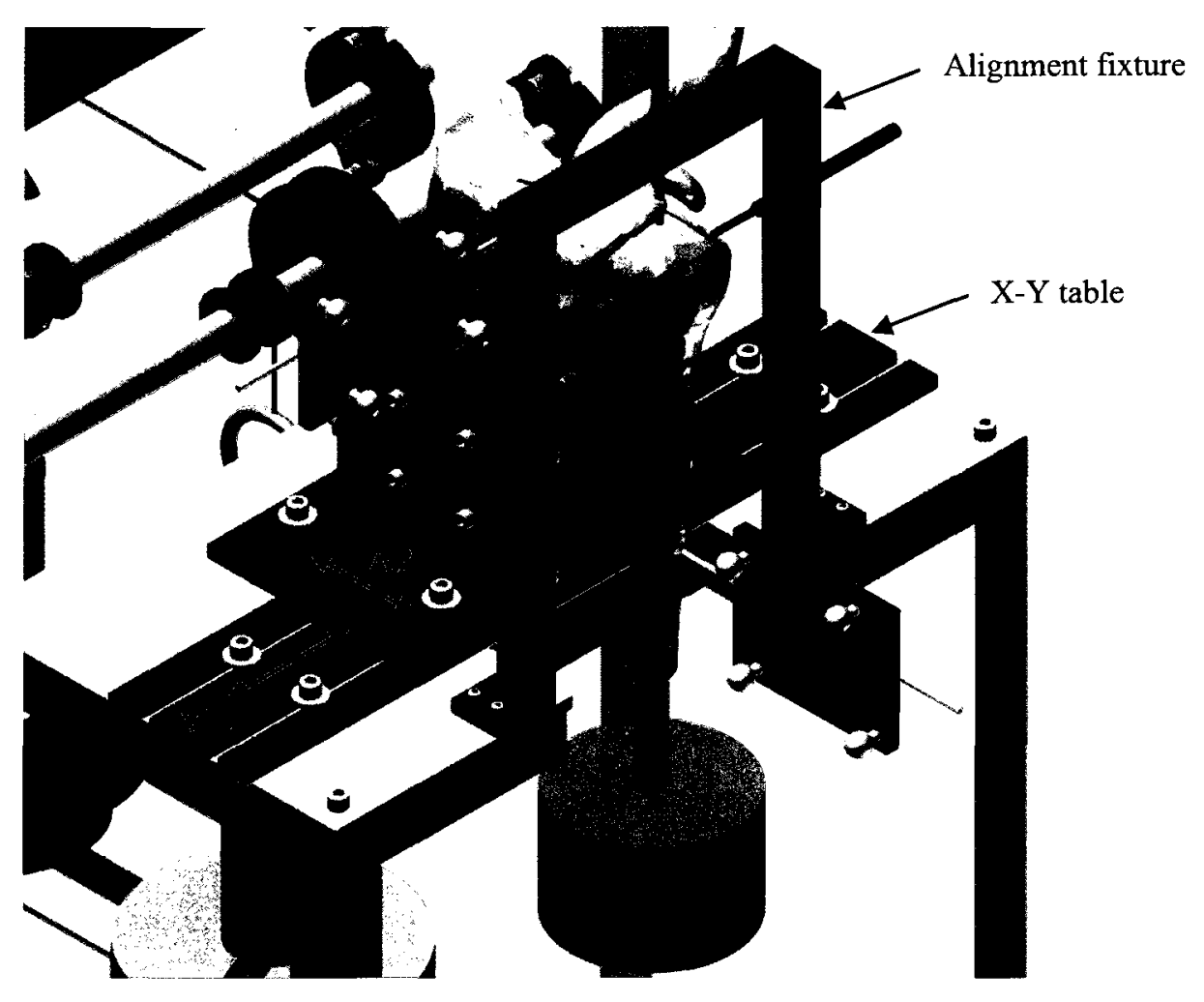

Figure 13: Alignment of Potting Cup in Shoulder Simulator with X-Y table.

To abduct the arm in the scapular plane, the cuff muscles are loaded with a constant ratio relative to the deltoid force (Table 2). The selected ratios represent the mean muscle force ratios of previously published simulators. ${ }^{2,32-34}$

Table 2: Constant rotator cuff muscle ratios.

\begin{tabular}{ll}
\hline Muscle & Ratio \\
\hline Deltoid & 1.00 \\
Supraspinatus & 0.18 \\
Infraspinatus and Teres Minor & 0.42 \\
Subscapularis & 0.40 \\
\hline
\end{tabular}


A cable attached to a bone screw at the deltoid insertion site applied the deltoid abductor force. The deltoid cable is aligned in the antero-posterior direction with the scapular plane. The location of the pulley is adjusted with slots in the medial-lateral and superior-inferior directions such that the line of action was closest to the acromion to reproduce the physiological moment arm of the deltoid (Figure 14). The deltoid cable runs through a series of pulleys mounted with needle bearings on shafts to reduce friction.
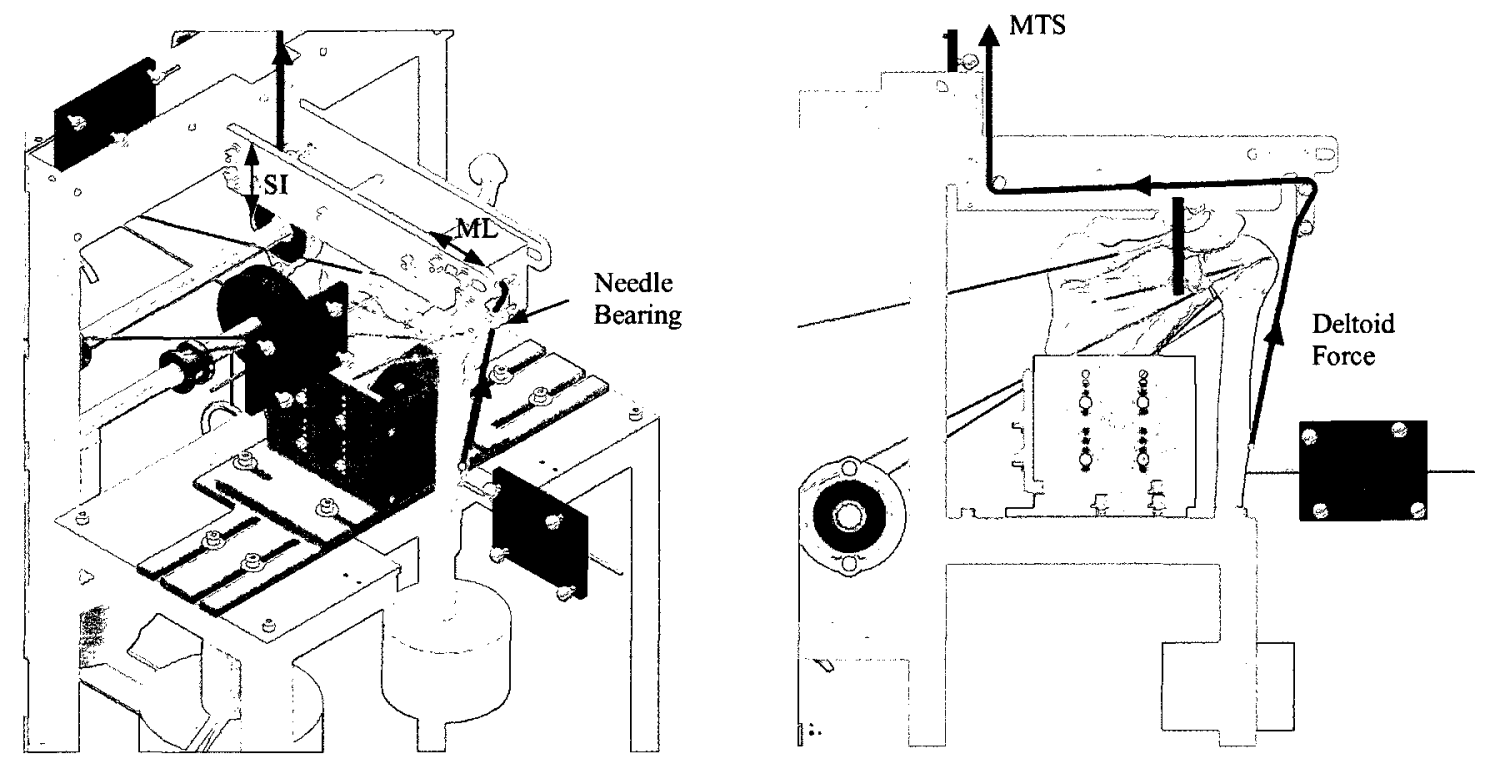

Figure 14: Deltoid cable activated by MTS load frame. Superior-inferior (SI) and medio-lateral (ML) adjustment completed with slots.

For each rotator cuff muscle, a pulley system is used to apply calibrated precision weights on a precision weight hanger (Troemner, Thorofare, NJ). Two pulleys are mounted on a stainless steel shaft rotating on bearings (eccentric collar locking type, SA202-10, KML Bearing Canada Inc, Dorval, QC) mounted to the side walls of the shoulder simulator (Figure 15). The alignment pulley is adjusted along the length of the shaft with set screws to align its center with the centroid of the respective muscle. The 
outer diameter of the weight and position pulley (Figure 16) is designed such that the ratios of the effective ratios (equation 1) respect the prescribed constant rotator cuff muscle ratios (Table 2). Taking advantage of the gear ratio (equation 2), equal weight is applied to each of the three hangers simplifying methodology during testing.

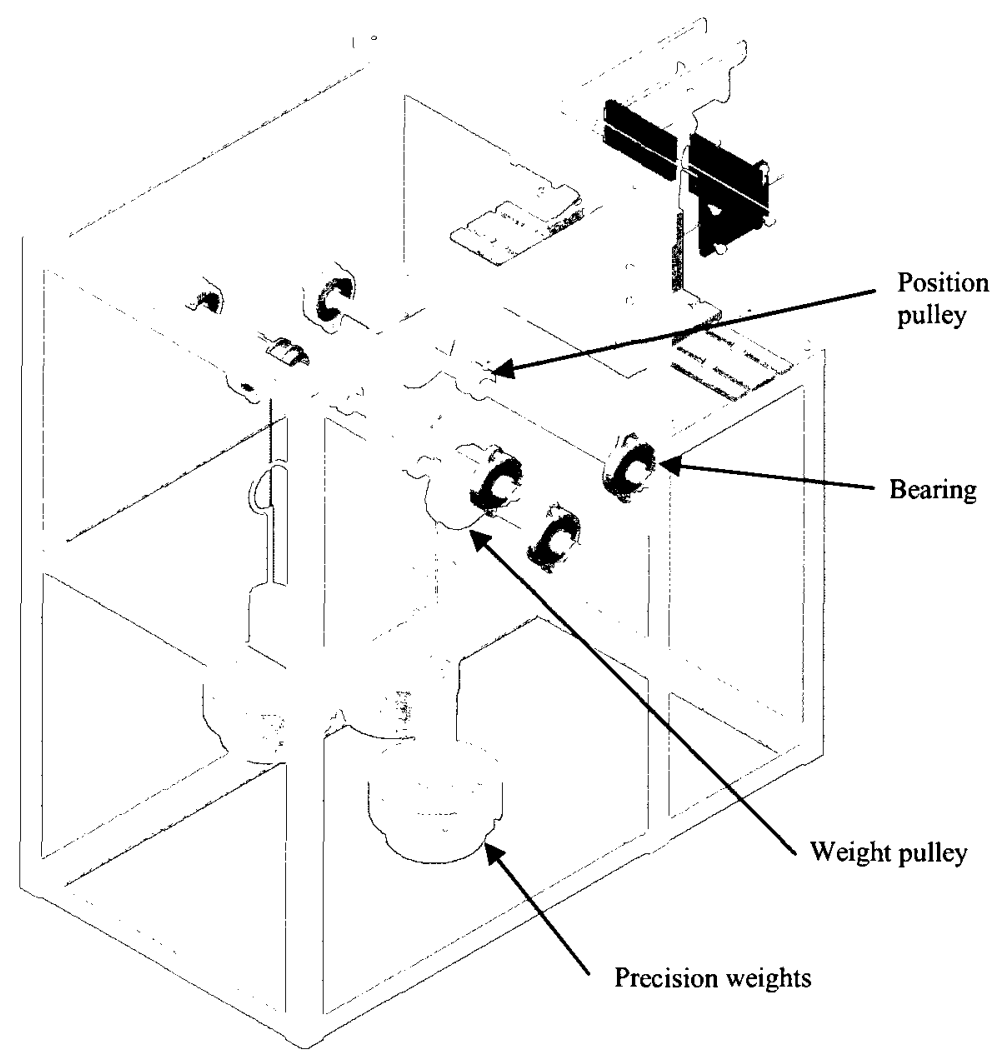

Figure 15: Static weights applied to rotator cuff: Supraspinatus (SSP), Subscapularis (SUB) and Infraspinatus (ISP). 


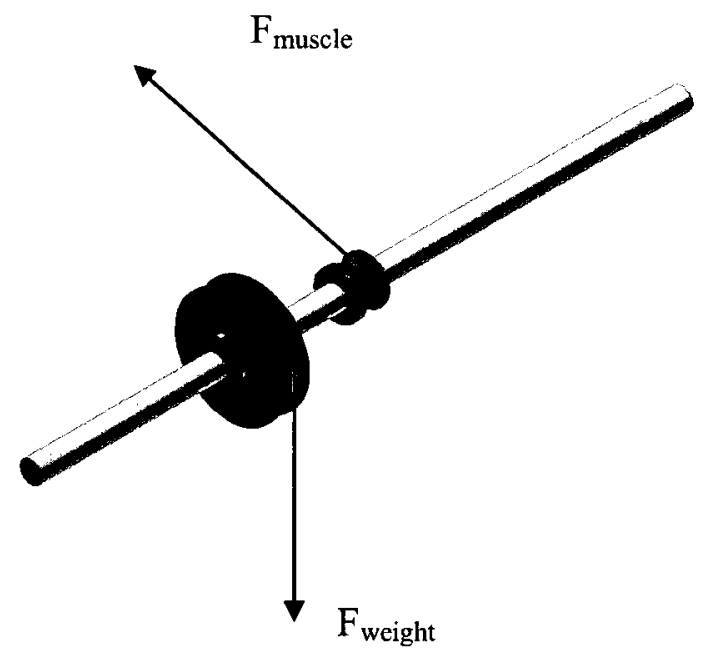

$$
\begin{aligned}
& E R=\frac{D_{\text {weight }}+D_{\text {cable }}}{D_{\text {position }}+D_{\text {cable }}} \text { (eq. 1) } \\
& F_{\text {muscls }}=E R \times F_{\text {weight }} \text { (eq. 2) }
\end{aligned}
$$

ER: effective ratio

$D_{\text {weight }}$ diameter of weight pulley

$\mathrm{D}_{\text {position: }}$ diameter of position pulley

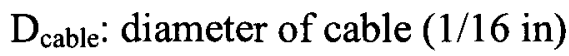

Figure 16: Rotator cuff static loading - effective gear ratio.

The cables attached to the subscapularis and infraspinatus/teres minor are aligned with the predetermined physiological lines of action with adjustable eyelets attached to the base of the potting cup (Figure 17). The physiological lines of action of each muscle are approximated during dissection by determining the center of mass of the muscle and marking this point on the medial border of the scapula. The positions of the rotator cuff pulleys in the simulator were designed to intersect published normalized physiological rotator cuff lines of action. ${ }^{48}$ Alignment for the supraspinatus relies on the position of the pulley. Given that the specimen is aligned in the simulator with respect to the center of the humeral head, inter-specimen variability of the line of action of the supraspinatus will be minimal. 


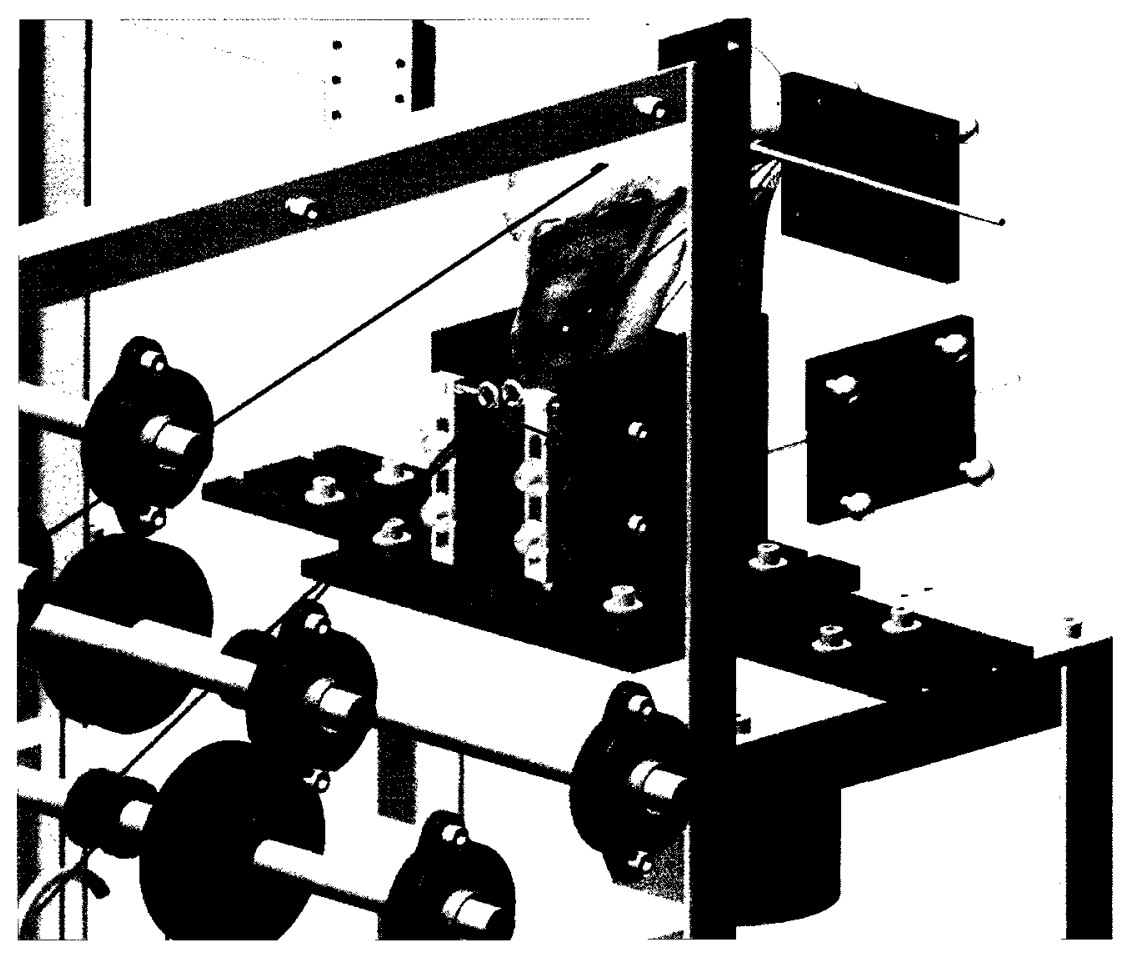

Figure 17: Adjustable eyelets align the rotator cuff cables with physiological lines of action. 


\subsection{Measurement of Subacromial Pressure}

To measure subacromial contact pressure, a K-scan pressure sensor (model 5051, Tekscan Inc, Boston, MA) was inserted into the subacromial space. The optimal sensor type was determined during pilot testing $(n=6)$. The sensing element has an array of 1936 sensors $(5.6 \mathrm{~cm}$ in width and $5.6 \mathrm{~cm}$ in depth) and minimal thickness $(0.1 \mathrm{~mm})$ which is important to minimize interference of the sensor with normal joint kinematics. The sensel density of the sensor is 62 sensels per square centimetre. The sensor was centered on the antero-lateral corner of the acromion. For each static position, the average and peak pressure as well as the force and contact area were computed using the I-scan software (Tekscan Inc, Boston, MA).

The sensor was calibrated as per the manufacturer's recommended procedure by applying two known compressive forces with a materials testing machine (MTS 858 Minibionix, MTS Systems Corp, Eden Prairie, MN). The sensor was compressed between two aluminum square fixtures $(30 \mathrm{~mm} \times 30 \mathrm{~mm})$ each lined with a synthetic rubber sheet approximately $1 \mathrm{~mm}$ in thickness (Figure 18). A ball bearing was used to apply the force to the square fixture to ensure that the force transmitted was normal resulting in a uniform pressure pattern. 


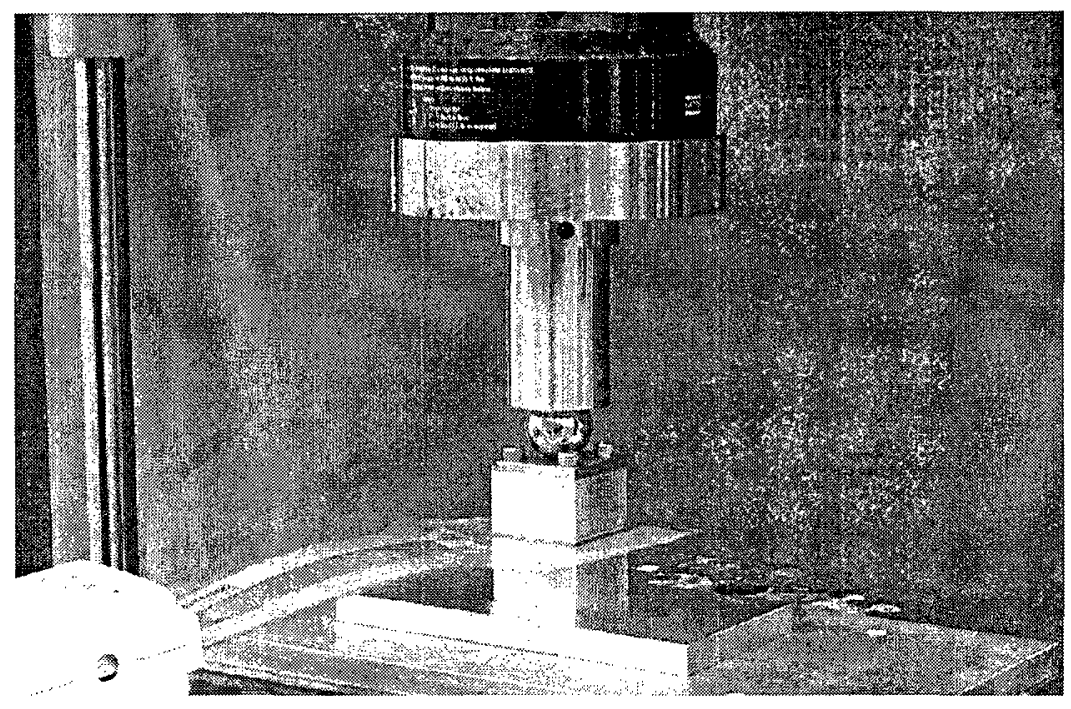

Figure 18: Tekscan Calibration Fixtures.

Two loads were applied during $30 \mathrm{~s}$ for sensor calibration: 20 and $80 \%$ of the saturation compressive load. The I-scan software applied a two-point power law regression of the form

$$
y=A x^{B}
$$

where

$$
\begin{aligned}
& y=\text { pressure }[\mathrm{kPa}] \\
& x=\text { raw sensor output }(0-255) \\
& A=\text { scale factor } \\
& B=\text { exponential factor }
\end{aligned}
$$

The calibration was assessed by applying four loads: $20,40,60$ and $80 \%$ of the saturation load. The computed force from the I-scan software was compared with the applied load with the MTS load cell. 


\subsection{Measurement of Glenoh umeral Kinematics}

Glenohumeral kinematics were measured with an optical tracking system (Polaris, NDI, Waterloo, ON) connected to a computer running MATLAB (The Mathworks ${ }^{\mathrm{TM}}$, Natick MA). Passive marker tools were designed (see Appendix C.2) following the minimum distance requirements defined by the manufacturer. The passive marker tools were drilled on both the scapula and the humerus. Landmarks were digitized to define the scapula and humerus coordinate systems following the ISB recommendation ${ }^{49}$ with modification to the scapula coordinate system: the acromioclavicular (AC) landmark replaced the acromial angle (AA) to align the scapular coordinate system with the scapular plane (Figure 19). The rotation of the humerus with respect to the scapula was decomposed in three Euler angles following the yxy convention: plane of abduction, abduction and axial rotation according to the ISB recommendation. ${ }^{49}$ The translation of the geometric center of the humeral head was computed in the scapular coordinate system.

For all the coordinate transforms, the following conventions are used to transform from coordinate system B to coordinate system A:

$$
\mathbf{P}_{\mathrm{A}}=\boldsymbol{R}_{\mathrm{BA}} \mathbf{P}_{\mathrm{B}}+\mathbf{T}_{\mathrm{A}} \text { (eq. 4) }
$$

where

$\mathbf{P}_{\mathrm{A}}$ and $\mathbf{P}_{\mathrm{B}}$ are $3 \times 1$ vectors

$\boldsymbol{R}_{\mathrm{BA}}$ is a $3 \times 3$ rotation matrix from B to $\mathrm{A}$

$\mathbf{T}_{\mathrm{A}}$ is a $3 \times 1$ translation from coordinate system $\mathrm{B}$ to $\mathrm{A}$ 

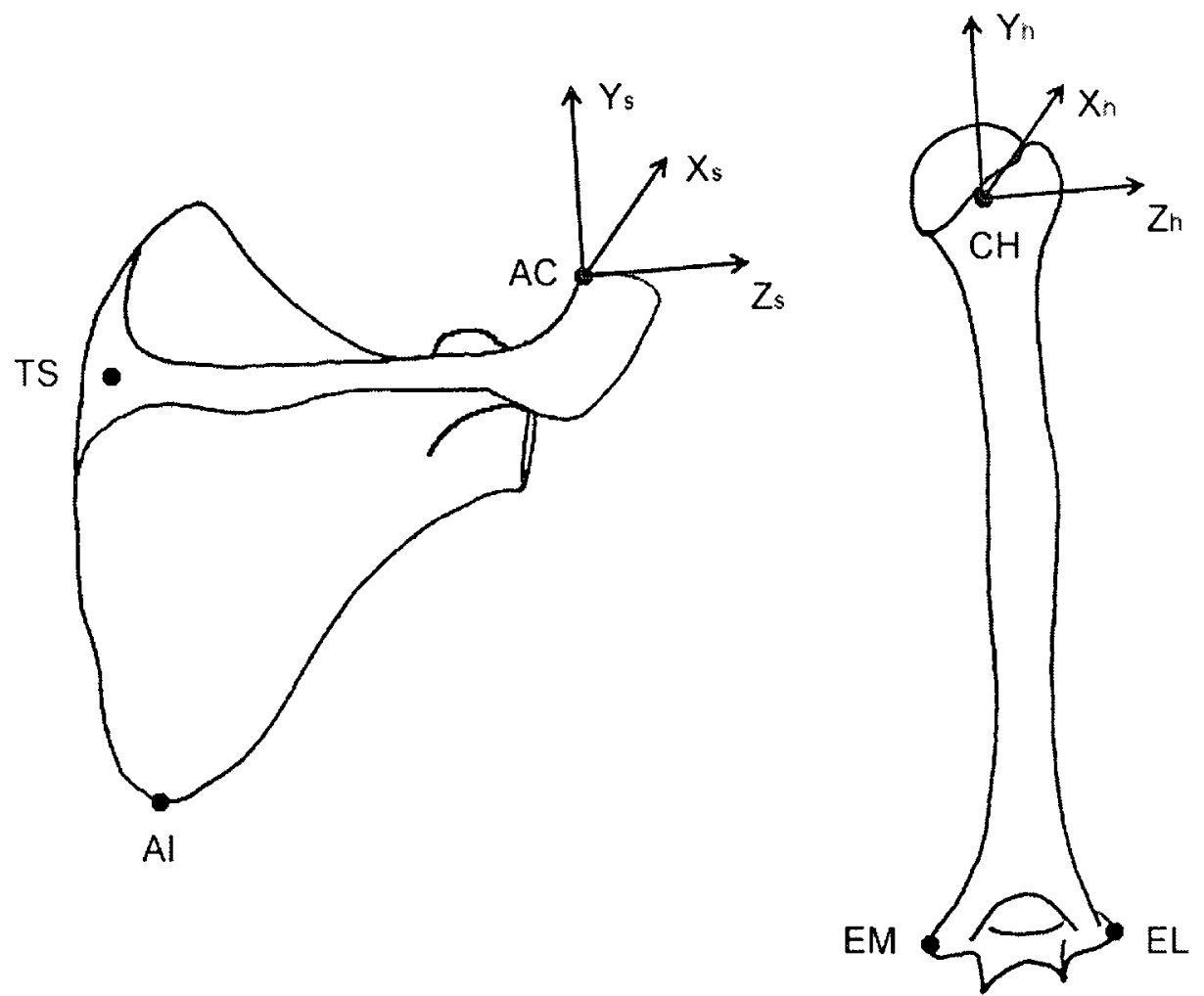

Figure 19: Definitions of Scapula and Humerus Coordinate Systems.

The scapula coordinate system was defined as follows:

${ }^{\mathrm{G}} \mathbf{J}_{\mathrm{S}}:\left({ }^{\mathrm{G}} \mathbf{T S}-{ }^{\mathrm{G}} \mathbf{A I}\right) /\left\|\left({ }^{\mathrm{G}} \mathbf{T S}-{ }^{\mathrm{G}} \mathbf{A I}\right)\right\|$

${ }^{\mathrm{G}} \mathbf{I}_{\mathrm{S}}$ : perpendicular to the scapular plane formed by ${ }^{\mathrm{G}} \mathbf{T S},{ }^{\mathrm{G}} \mathbf{A I}$ and ${ }^{\mathrm{G}} \mathbf{A C}$ pointing forward

${ }^{\mathrm{G}} \mathbf{K}_{\mathrm{S}}$ : perpendicular to ${ }^{\mathrm{G}} \mathbf{I}_{\mathrm{S}}$ and ${ }^{\mathrm{G}} \mathbf{J}_{\mathrm{S}}$

Where:

${ }^{\mathrm{G}} \mathrm{AC}$ is the most dorsal point on the acromioclavicular joint in global coordinates

${ }^{\mathrm{G}}$ TS is the spinae scapulae in global coordinates

${ }^{\mathrm{G}} \mathbf{A I}$ is the inferior angle in global coordinates

${ }^{\mathrm{G}} \mathbf{I}_{\mathrm{S}},{ }^{\mathrm{G}} \mathbf{J}_{\mathrm{S}},{ }^{\mathrm{G}} \mathbf{K}_{\mathrm{S}}$ are $3 \times 1$ unit vectors 
The rotation matrix for the transformation from the scapula coordinate system to the global coordinate system is given by:

$$
\boldsymbol{R}_{\mathrm{SG}}=\left[{ }^{\mathrm{G}} \mathbf{I}_{\mathrm{S}}{ }^{\mathrm{G}} \mathbf{J}_{\mathrm{S}}{ }^{\mathrm{G}} \mathbf{K}_{\mathrm{S}}\right]
$$

To define the humerus coordinate system, the surface profile of the entire articulating surface of the humeral head was traced with a digitizing probe at the end of the test. The geometric center of the humeral head was determined using a least-squares sphere-fitting routine to a minimum of four hundred data points.

The humerus landmarks in global coordinates are transformed in the scapula coordinate system using the rotation matrix $\boldsymbol{R}_{S G}$ and equation 4 . The humerus coordinate system was defined as follows:

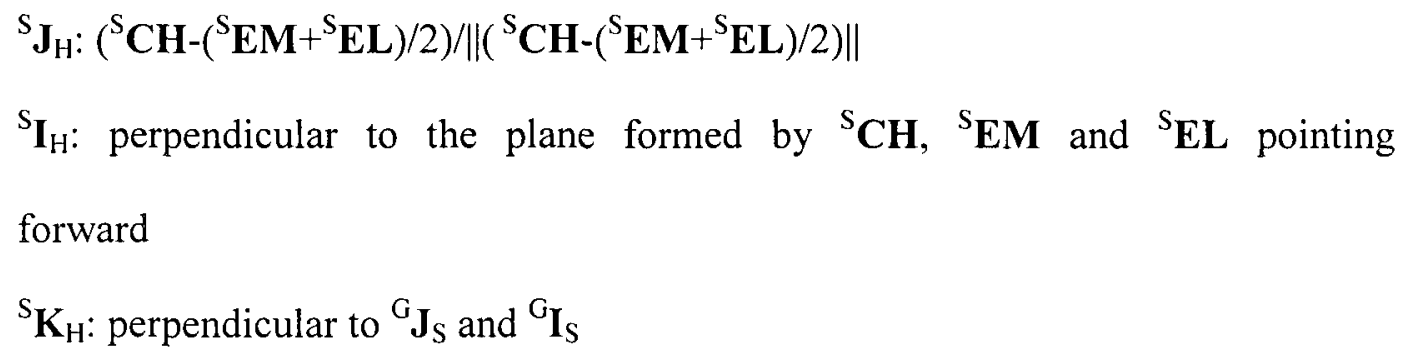

${ }^{\mathrm{S}} \mathbf{E M}$ is the medial epicondyle in the scapula coordinate system

${ }^{\mathrm{S}} \mathbf{E L}$ is the lateral epicondyle in the scapula coordinate system

${ }^{\mathrm{S}} \mathrm{CH}$ is the geometric center of the humeral head in the scapula coordinate system 
The rotation matrix for the transformation from the humerus coordinate system to the scapula coordinate system is given by:

$$
\boldsymbol{R}_{\mathrm{HS}}=\left[{ }^{\mathrm{S}} \mathbf{I}_{\mathrm{H}}{ }^{\mathrm{S}} \mathbf{J}_{\mathrm{H}}{ }^{\mathrm{S}} \mathbf{K}_{\mathrm{H}}\right]
$$

The three angles of glenohumeral rotation following the yxy Euler convention are determined with equation 5 :

$$
=\left[\begin{array}{ccc}
\cos \beta_{1} \cos \beta_{3}-\sin \beta_{1} \cos 2 \sin \beta_{3} & \sin \beta_{1} \sin \beta_{2} & \cos \beta_{1} \sin \beta_{3}-\sin \beta_{1} \cos \beta_{4} \cos \beta_{3} \\
\sin \beta_{2} \sin \beta_{3} & \cos \beta_{2} & -\sin \beta_{2} \cos \beta_{3} \\
-\sin \beta_{1} \cos \beta_{3}-\cos 1 \cos \beta_{2} \sin \beta_{3} & \cos \beta_{1} \sin \beta_{2} & -\sin \beta_{1} \sin \beta_{3}-\cos \beta_{1} \cos \beta_{2} \cos \beta_{3}
\end{array}\right]
$$

Where

$\beta_{1}$ is the angle between the plane of abduction and the scapular plane

$\beta_{2}$ is the angle of abduction

$\beta_{3}$ is the axial rotation

The geometric center of the humeral head in the scapular coordinate system $\left({ }^{\mathrm{S}} \mathbf{C H}\right)$ is computed for each shoulder position using equation 4 and the rotation matrix, $\boldsymbol{R}_{S \mathrm{G}}$. Translation of the humeral head is defined as the displacement of its geometric center from the initial (or reference) position. 
To obtain real-time data for the neutral alignment of the humerus, the center of the humeral head was approximated using a regression of anatomical landmarks of the scapula. ${ }^{50}$ In the reference position, the axial rotation of the humerus was calculated as the angle between ${ }^{S} \mathbf{K}_{\mathrm{H}}$ and the scapular plane formed by ${ }^{\mathrm{S}} \mathbf{T S},{ }^{\mathrm{S}} \mathbf{A I}$ and ${ }^{\mathrm{S}} \mathbf{A C}$ to avoid problems associated with Euler angles near zero degrees of abduction ${ }^{51}$ :

$$
\mathbf{n} \cdot{ }^{\mathrm{s}} \mathbf{K}_{\mathrm{H}}=|\mathbf{n}|{ }^{\mathrm{S}} \mathbf{K}_{\mathrm{H}} \mid \sin \alpha \quad \text { (eq. 6) }
$$

where

$\mathrm{n}=\left[\begin{array}{lll}1 & 0 & 0\end{array}\right]$

${ }^{\mathrm{S}} \mathbf{K}_{\mathrm{H}}$ is a unit vector parallel to the medial and lateral epicondyles

$a$ is the axial rotation of the humerus 


\subsection{Study Protocol}

Ten fresh-frozen human cadaveric shoulders from seven donors (mean age: $72 \pm$ 9; range $57-80 ; 4$ male; 6 right) were obtained following ethics approval from the Ottawa Hospital Research Ethics Board (OHREB). All experiments were conducted at the Orthopaedic Biomechanics Laboratory located at the CHEO Research Institute. Specimens were excluded from the study if there was any evidence of osteoarthritis or full thickness cuff tears on visualization during arthroscopy. Arthroscopy was performed on all specimens prior to removal of skin and subcutaneous tissue. Specimens were stored at -20 degrees and thawed 24 hours prior to testing.

The shoulder including the entire scapula and humerus as well as the clavicle transected at its mid-point was dissected prior to mounting in the shoulder simulator. The specimen was kept moist throughout testing with a normal saline spray. All soft tissues except for the rotator cuff, joint capsule, coracoacromial and glenohumeral ligaments were removed. The deltoid was carefully dissected and the centroid of the broad insertion on the humeral shaft was marked. Once the rotator cuff was exposed, muscle bellies of the supraspinatus, the infraspinatus, the teres minor and the subscapularis were detached from the scapula. The centroids of the subscapularis and the combined infraspinatus and teres minor were approximated and marked on the scapula near the medial border for later alignment in the simulator. All rotator cuff muscles were transected $1 \mathrm{~cm}$ medial to their musculotendinous junctions and attached to cables with number 2 braded polyblend sutures (Herculine ${ }^{\mathrm{TM}}$, Linvatec Corp, Largo, Florida). A whip stitch was used to grasp the full width of the tendon. 
To tighten the posterior capsule, zero monofilament suture (PDS II, Ethicon,) was used in a running simple stitch to close the capsule medio-laterally following a method previously described by Gerber et al. ${ }^{47}$ In their study, the plication of the anterior or posterior capsule spanned a medio-lateral distance of $1 \mathrm{~cm}$.

In this study, three treatment groups were tested: no plication, $1 \mathrm{~cm}$ plication and $2 \mathrm{~cm}$ plication. The $1 \mathrm{~cm}$ plication was prepared lateral from the centerline at a distance of $1 \mathrm{~cm}$. To achieve the $2 \mathrm{~cm}$ plication, an additional $1 \mathrm{~cm}$ plication was completed $1 \mathrm{~cm}$ medial from the centerline (Figure 20). Sutures were inserted prior to testing without tightening.

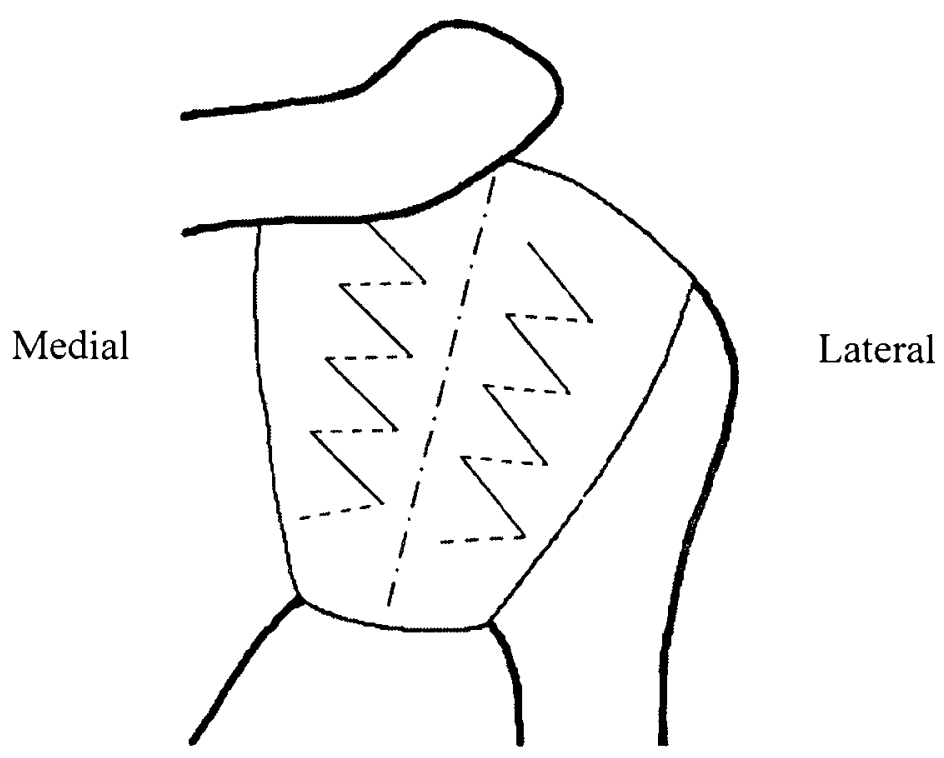

Figure 20: Schematic representation of posterior capsular plication.

The dissected shoulder was mounted on the custom designed shoulder simulator (Figure 11) adapted from previously published cadaveric shoulder simulators. ${ }^{2,32-34}$ The humerus was transected $20 \mathrm{~cm}$ from the center of the humeral head. A $3.5 \mathrm{~kg}$ weight was 
rigidly attached $30 \mathrm{~cm}$ distal to the center of the humeral head with a cemented intramedullary rod to simulate the effective ( $5 \%$ of average body weight) arm weight.,32

Prior to transecting the humerus, the positions of the medial and lateral epicondyles were recorded with a digitizing probe and the optical tracking system (Polaris, NDI, Waterloo, ON) for later definition of the humerus coordinate system. All landmarks on the scapula (see section 4.3 for details) were recorded prior to fixing the scapula to the simulator.

The bottom one third of the scapula was fixed in bismuth such that the plane of the scapula was parallel to the simulator and the medial border was vertical. The proper alignment of the scapula was verified with the passive tracking system prior to potting.

In the simulator, the cables attached to the subscapularis and infraspinatus/teres minor were aligned with the predetermined physiological lines of action with adjustable eyelets attached to the base of the potting cup. The eyelets were adjusted in the superiorinferior and antero-posterior directions to align the cables attached to the subscapularis and infraspinatus/teres minor with the predetermined physiological lines of action.

In the initial position, the humerus was aligned in neutral rotation with $45 \mathrm{~N}$ applied to the deltoid with associated rotator cuff loads to prevent subluxation. Neutral rotation was defined as the anatomical neutral position in which the epicondylar axis is parallel with the coronal plane. ${ }^{52}$ Given that the scapular plane is approximately 30 degrees posterior to the coronal plane $\mathrm{e}^{28}$, the epicondylar axis was externally rotated 30 degrees with respect to the scapular plane. To obtain real-time data for kinematics, the center of the humeral head was approximated using a regression of anatomical landmarks of the scapula. ${ }^{50}$ 
Three preliminary trials of humeral abduction to approximately 90 degrees of glenohumeral abduction with respect to vertical (approximately 135 degrees of clinical abduction) were completed by sequentially increasing the deltoid force by $30 \mathrm{~N}$ increments. The three preliminary trials were used to determine the approximate position of peak pressure. For each static position, the deltoid load was held for 30 seconds during which the subacromial pressure and glenohumeral kinematics were recorded. Prior to commencing the next loading ramp, the cuff loads were adjusted with static weights to reflect the end position of the next iteration.

The sequence of test conditions (no plication, $1 \mathrm{~cm}$ and $2 \mathrm{~cm}$ plication) was randomly assigned to each specimen (see Appendix A for randomization schedule). When necessary, a plication was released by cutting and removing the suture. A new suture was used to recreate the released plication. This way, for all test conditions, the sutures were present but not necessarily tightened.

For each condition, the humerus was abducted to one increment $(30 \mathrm{~N})$ prior to the position of peak pressure as determined from the preliminary trials. From this position, the deltoid was sequentially increased by $10 \mathrm{~N}$ increments for seven iterations. Thus, seven positions with $10 \mathrm{~N}$ increments spanning across the preliminary position of peak pressure were tested following the same procedure as the preliminary trials. Each position was held for 30 seconds and the subacromial contact pressure and kinematics were recorded. A minimum of two repeated trials were completed for each condition. 


\subsection{Data Analysis}

Repeatability of the shoulder simulator as measured by the Euler angles as well as the repeatability of the pressure measurements were computed as the average of the range of repeated trials at the position of peak pressure.

The average and peak subacromial pressure as well as the glenohumeral kinematics at the position of peak pressure were averaged for repeated trials. Treatment groups (no plication, $1 \mathrm{~cm}$ and $2 \mathrm{~cm}$ plication) were compared using a one-way repeatedmeasures analysis of variance (ANOVA) with a level of significance set at $p<0.05$. Posthoc comparisons were completed using a paired t-test with Bonferoni correction. Statistical analysis was performed using SPSS version 14 (SPSS Inc., Chicago, IL). 


\section{Chapter 5: Results}

Eight specimens were included in the final analysis of subacromial contact pressure. One specimen was excluded because the contact pressure exceeded the saturation level of the sensor while a second specimen was excluded as it exhibited no subacromial contact pressure throughout the full arc of abduction. For kinematic analysis, seven specimens were included: the same two specimens were excluded because the position of peak pressure could not be determined and a third specimen was excluded because a tool marker shifted during testing.

\subsection{Repeatability of Shoulder Simulator}

Angular repeatability (average of the range \pm standard deviation of the range) was $1.7 \pm 1.5$ degrees, $1.3 \pm 0.7$ degrees and $1.2 \pm 0.8$ degrees for abduction, plane of abduction and rotation respectively at the position of peak pressure. Repeatability of humeral head translation at the position of peak pressure was $0.5 \pm 0.4 \mathrm{~mm}$ and $0.6 \pm 0.6 \mathrm{~mm}$ for superior and anterior translation respectively. The repeatability of subacromial contact measurements was $58 \pm 59 \mathrm{kPa}, 12 \pm 15 \mathrm{kPa}, 2.0 \pm 3.0 \mathrm{~N}$ and $27 \pm 17 \mathrm{~mm}^{2}$ for peak pressure, average pressure, force and area respectively. 


\subsection{Tekscan Calibration}

Five model 5051 sensors were used to measure subacromial pressure in this study. Calibration factors for each of the five sensors are shown in Table 3.

Table 3: Calibration factors for calibration $\left(y=A x^{b}\right)$ of Tekscan sensors (model 5051$)$. ${ }^{*}$ exceeded saturation pressure.

\begin{tabular}{cccccc}
\hline Sensor & Range (psi) & \multicolumn{2}{c}{ Calibration Factor } & Saturation & Specimens \\
& & A & B & Pressure (kPa) & \\
\hline A & 75 & 0.053 & 1.431 & 1009 & $1,2^{*}$ \\
B & 150 & 0.162 & 1.265 & 1233 & 4 \\
C & 150 & 0.176 & 1.256 & 1279 & 5,6 \\
D & 150 & 0.111 & 1.350 & 1353 & 7,8 \\
E & 150 & 0.068 & 1.453 & 1464 & 9,10 \\
\hline
\end{tabular}

Subacromial pressure measurements in the second specimen exceeded the saturation of the sensor near the peak pressure position. A 5051 sensor with higher saturation pressure was ordered for following specimens. Specimen three was tested with sensor model 4201. Sensors are re-usable but the sensor was changed when it was either damaged or kinked following testing. Figure 21 illustrates the calibration curves for all 150 psi sensors. The percent error between the computed load and the applied load during verification of the calibration varied between 2 and $3 \%$. 


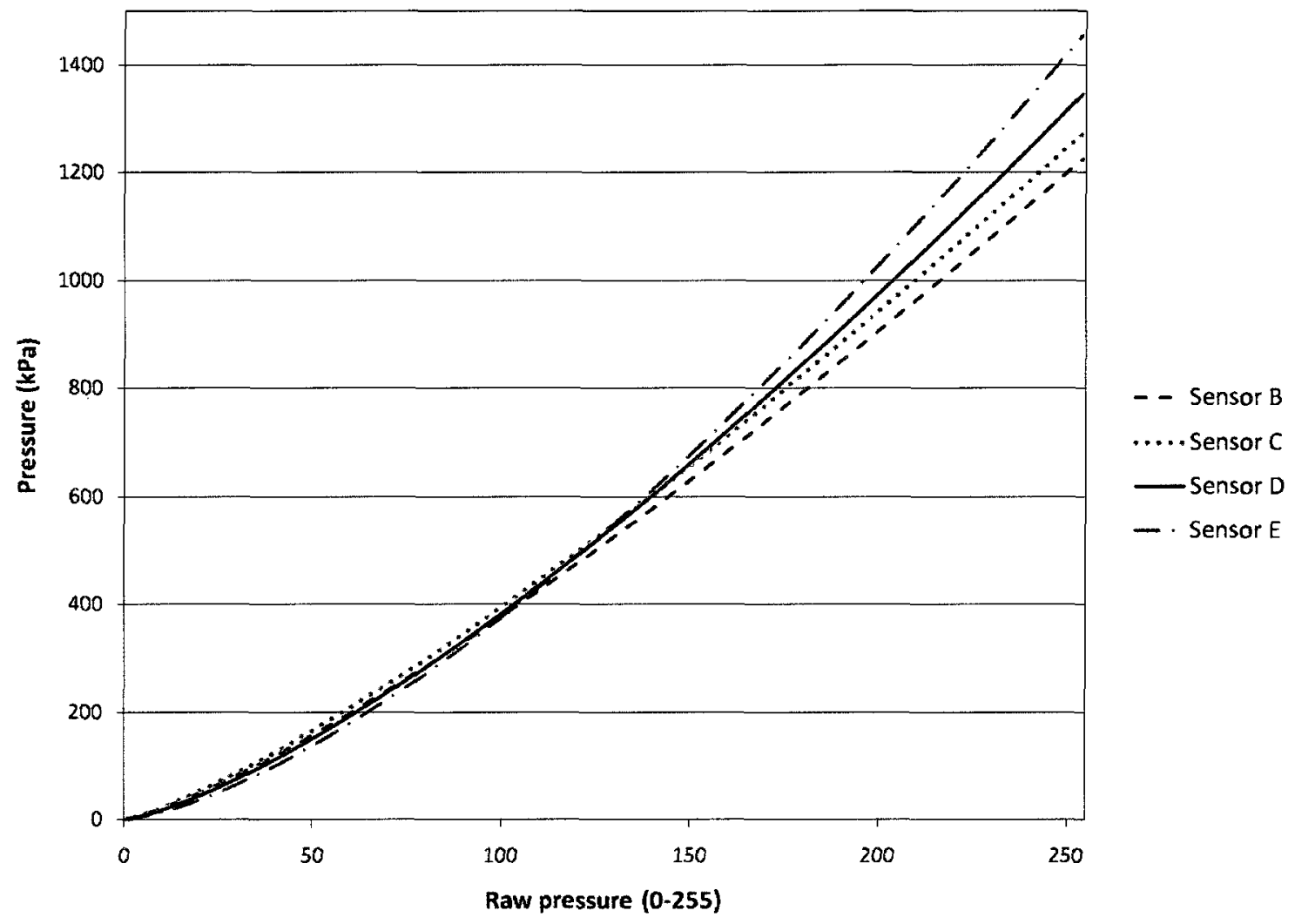

Figure 21: Calibration curves $\left(y=A x^{b}\right)$ plotted for 150 psi sensors. 


\subsection{Subacromial Contact Pressure}

The area of peak pressure was located near the antero-lateral corner of the acromion. A typical subacromial pressure map is shown in Figure 22.

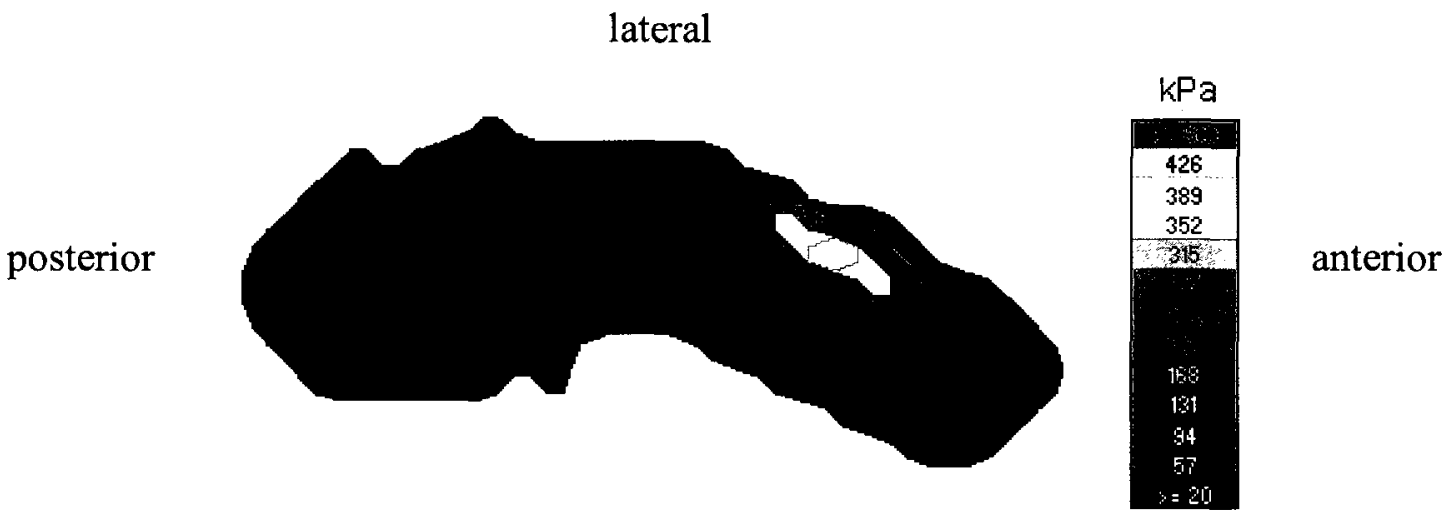

Figure 22: Typical subacromial pressure map.

Average peak subacromial contact pressure measured during the pre-test trials as a function of glenohumeral abduction angle is shown in Figure 23 for each specimen. The effect of plication on maximum peak subacromial contact pressure for each specimen is shown in Figure 24. Peak subacromial pressure data plotted against glenohumeral abduction angle for each condition and specimen is included in Appendix B. 


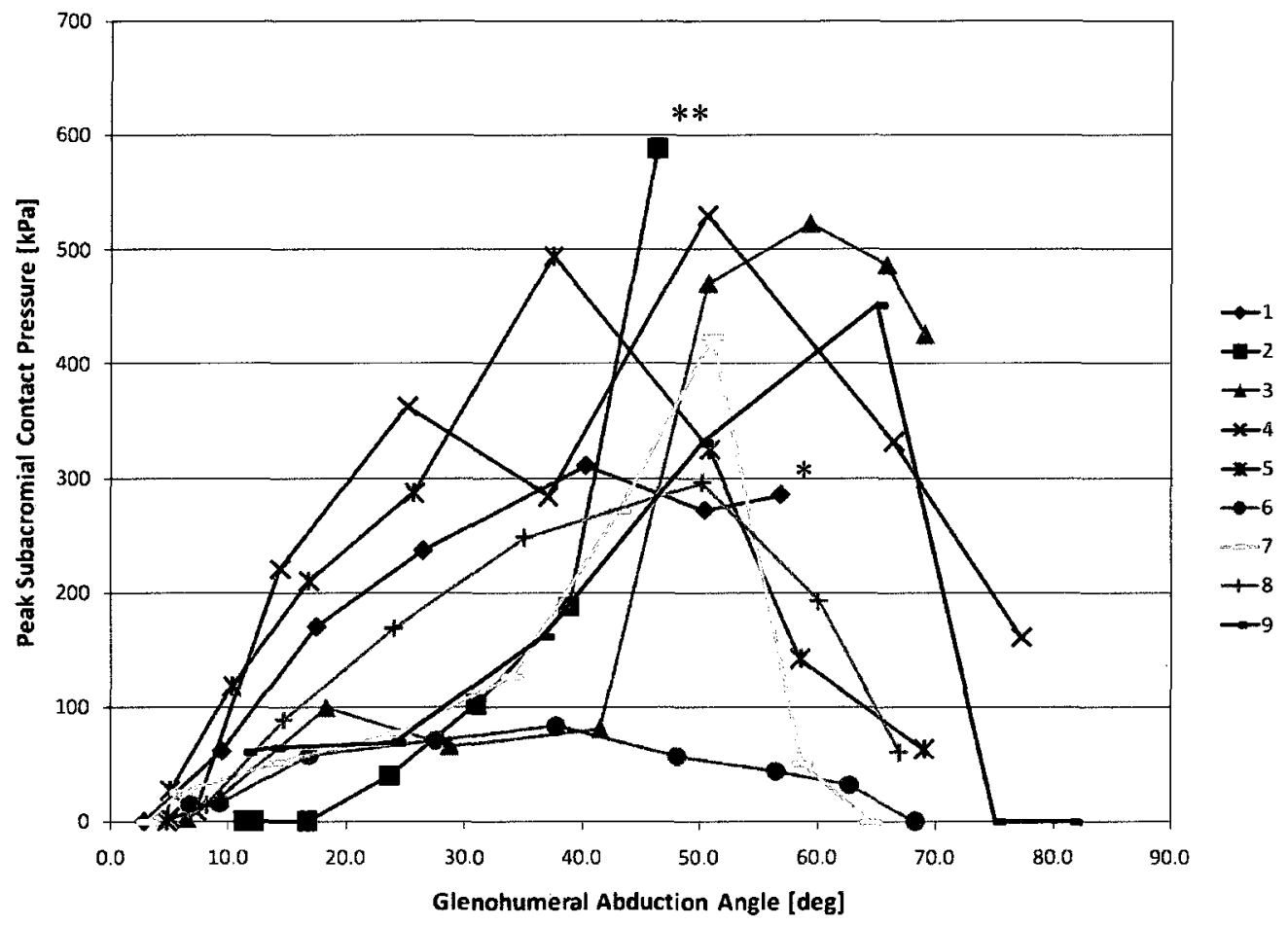

Figure 23: Peak subacromial contact pressure versus glenohumeral abduction angle for each specimen (pre-test data). * subacromial contact pressure after 60 degrees reached saturation due to abnormal bump on greater tubercle. ** specimen 2 reached saturation pressure of sensor.

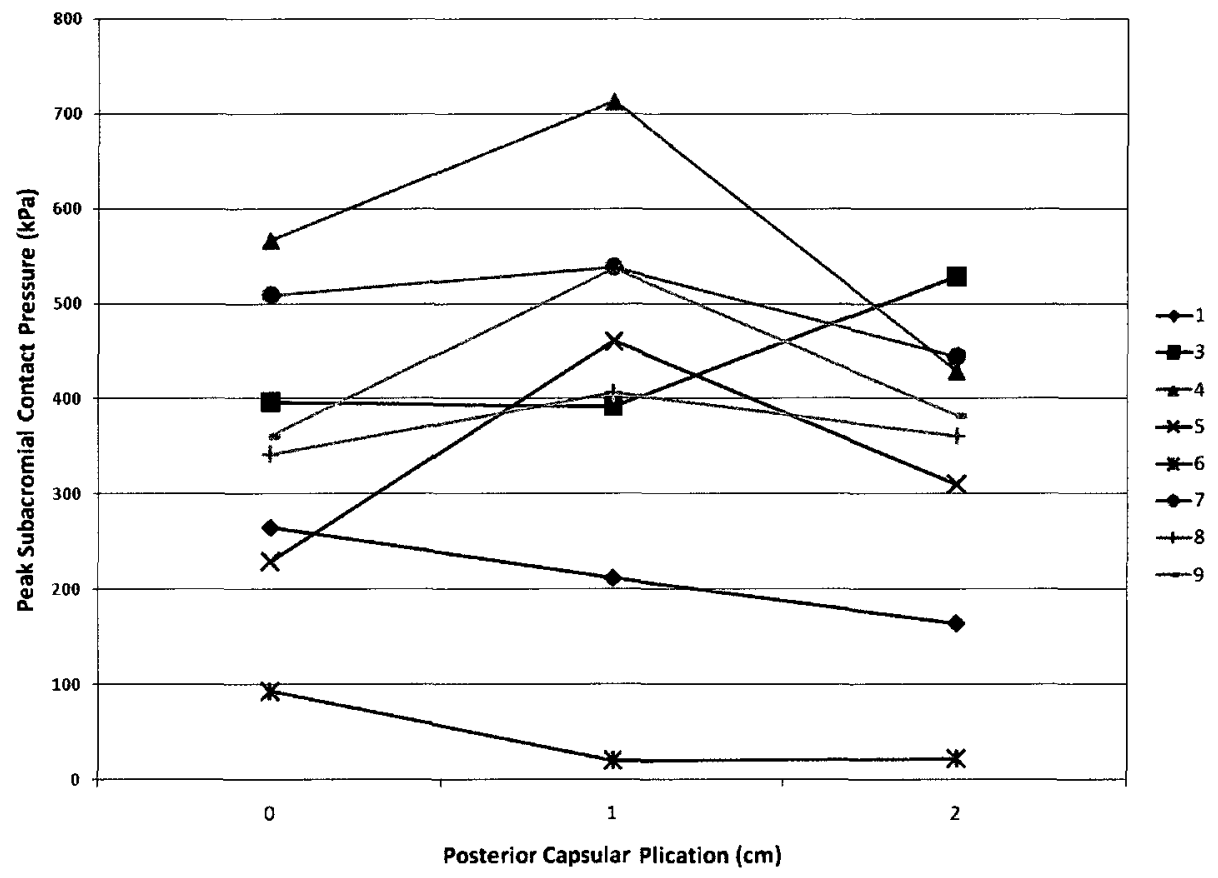

Figure 24: Effect of posterior capsular plication on peak subacromial pressure shown for each specimen. 
Posterior capsular plication did not result in a statistically significant increase in peak or average subacromial pressure (Figure 25) at the peak pressure position as determined by the repeated measures ANOVA $(p>0.05)$. Similarly, no significant difference was found for mean contact area or force (Table 4).

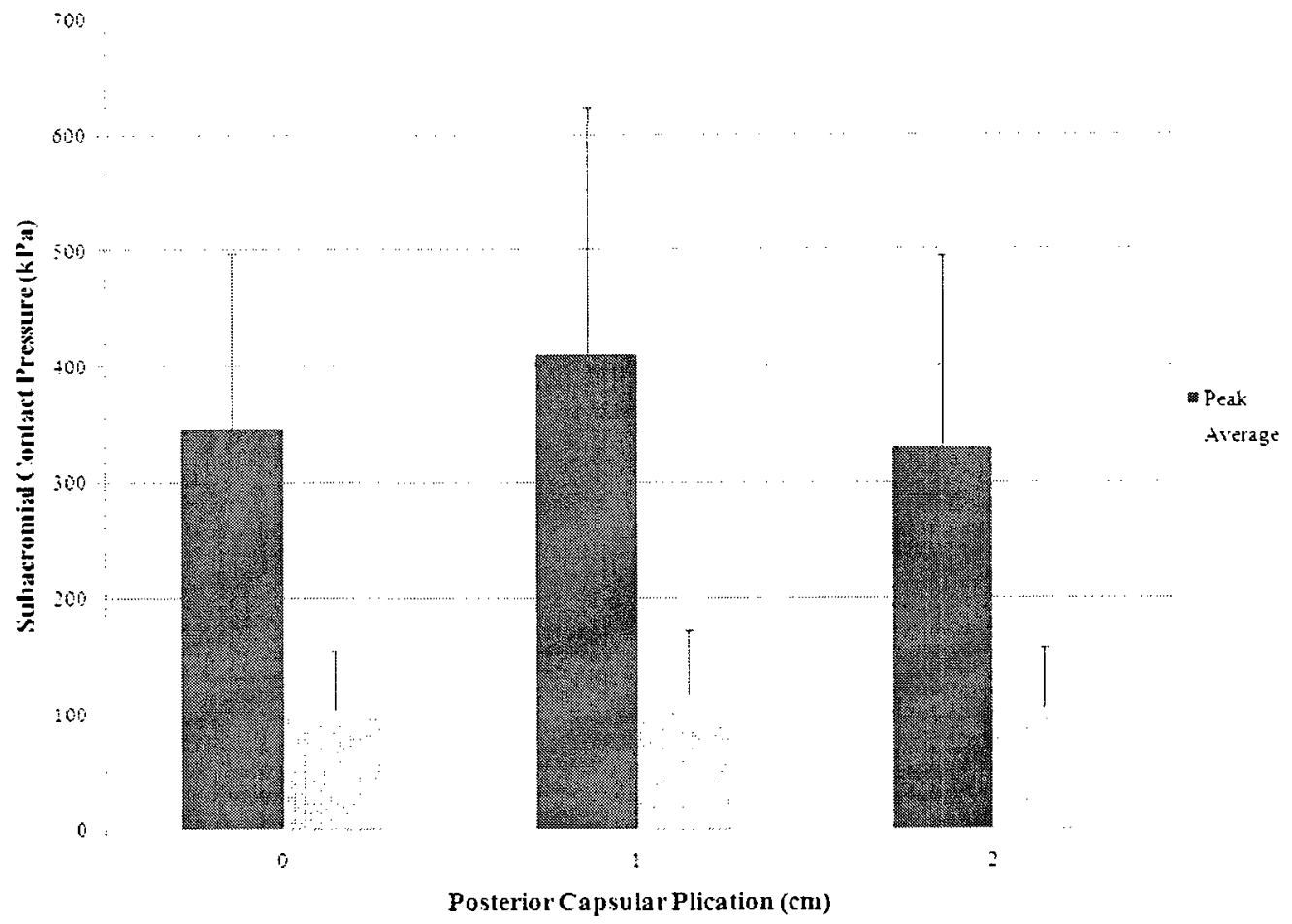

Figure 25: Peak and average (mean \pm sd) subacromial contact pressure for no plication, $1 \mathrm{~cm}$ and 2 $\mathrm{cm}$ plication of the posterior capsule $(\mathrm{n}=8)$. 
Table 4: Subacromial contact (peak and average pressure, force and area) averaged (mean \pm sd) for all specimens at the position of peak pressure throughout abduction cycle $(n=8)$.

\begin{tabular}{ccccc}
\hline Plication & $\begin{array}{c}\text { Peak } \\
\text { Pressure } \\
(\mathbf{k P a})\end{array}$ & $\begin{array}{c}\text { Subacromial Contact } \\
\text { Average } \\
\text { Pressure } \\
(\mathbf{k P a})\end{array}$ & $\begin{array}{c}\text { Force } \\
\mathbf{( N )}\end{array}$ & $\begin{array}{c}\text { Area } \\
\left(\mathbf{m m}^{2}\right)\end{array}$ \\
\hline \multirow{2}{*}{ None } & 345 & 104 & 11 & 149 \\
& $(152)$ & $(51)$ & $(6)$ & $(52)$ \\
\hline \multirow{2}{*}{$1 \mathrm{~cm}$} & 410 & 115 & 14 & 145 \\
& $(213)$ & $(56)$ & $(9)$ & $(67)$ \\
\hline \multirow{2}{*}{$2 \mathrm{~cm}$} & 330 & 105 & 11 & 141 \\
& $(164)$ & $(52)$ & $(7)$ & $(60)$ \\
\hline
\end{tabular}




\subsection{Glenohumeral Kinematics}

The plane of abduction and humeral axial rotation as well as the superior and anterior translation of the humeral head are plotted for each specimen as a function of glenohumeral abduction angle in Figures 26, 27, 28 and 29. 


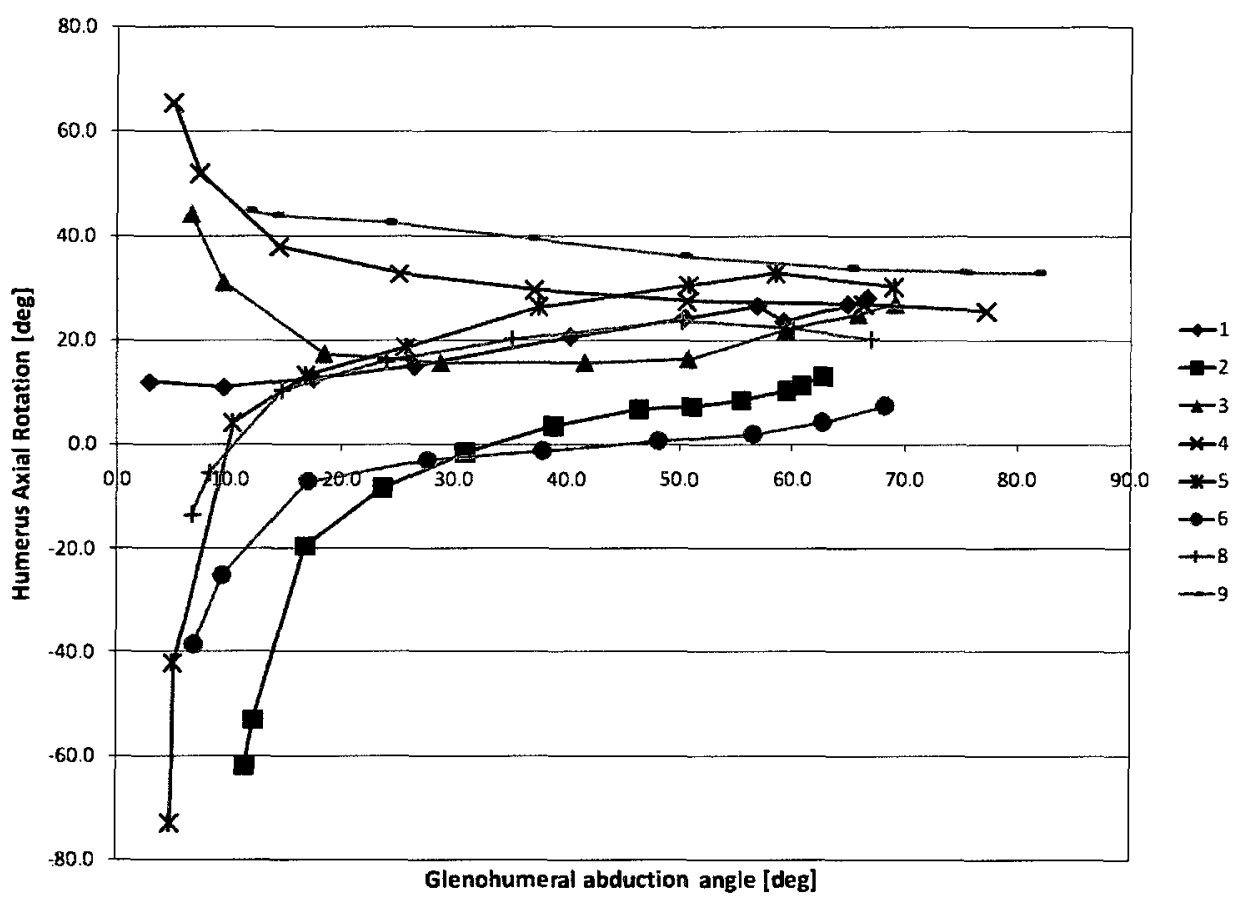

Figure 26: Humerus axial rotation (deg) versus glenohumeral abduction angle (deg) for each specimen (pre-test data).

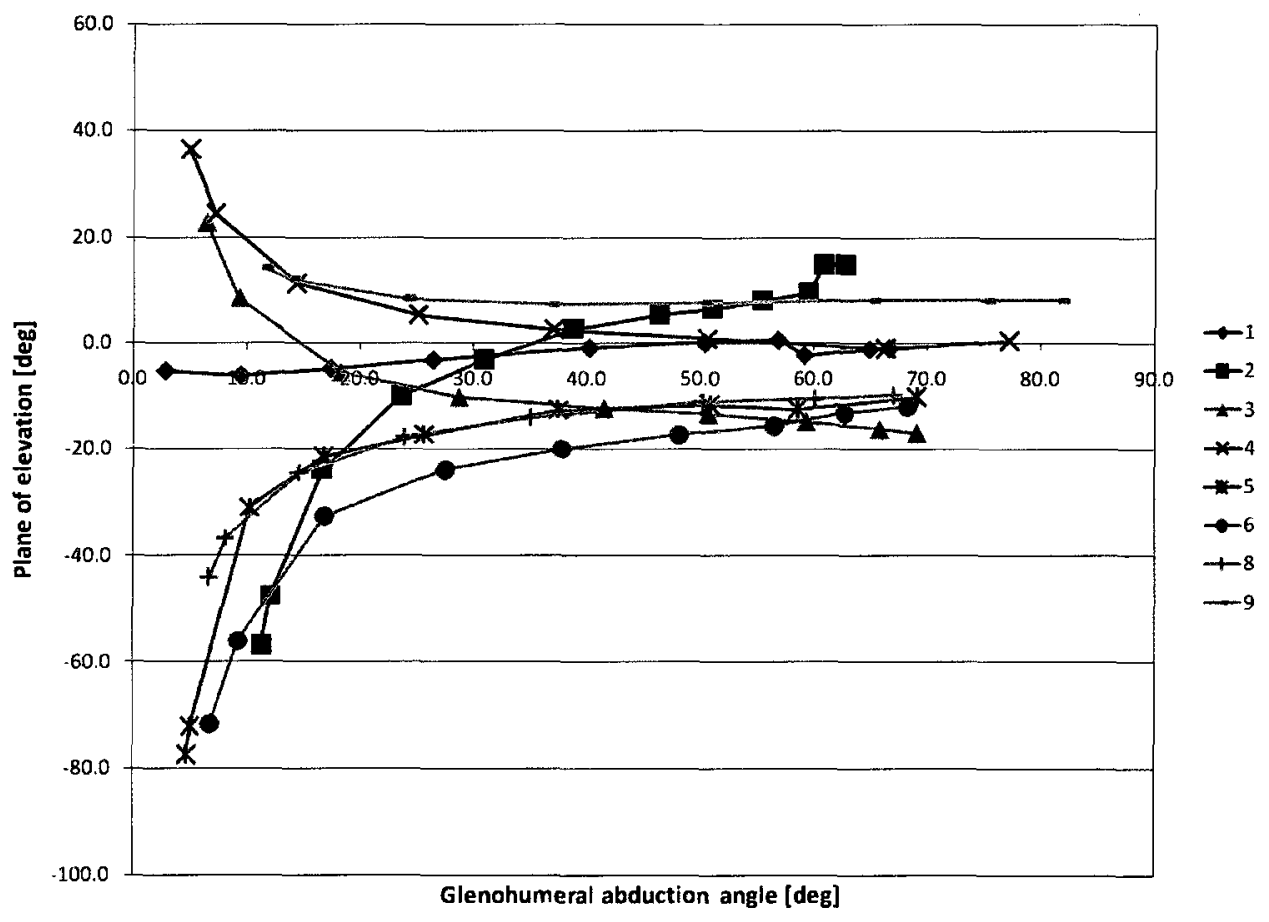

Figure 27: Plane of elevation (deg) versus glenohumeral abduction angle (deg) for each specimen (pre-test data). 


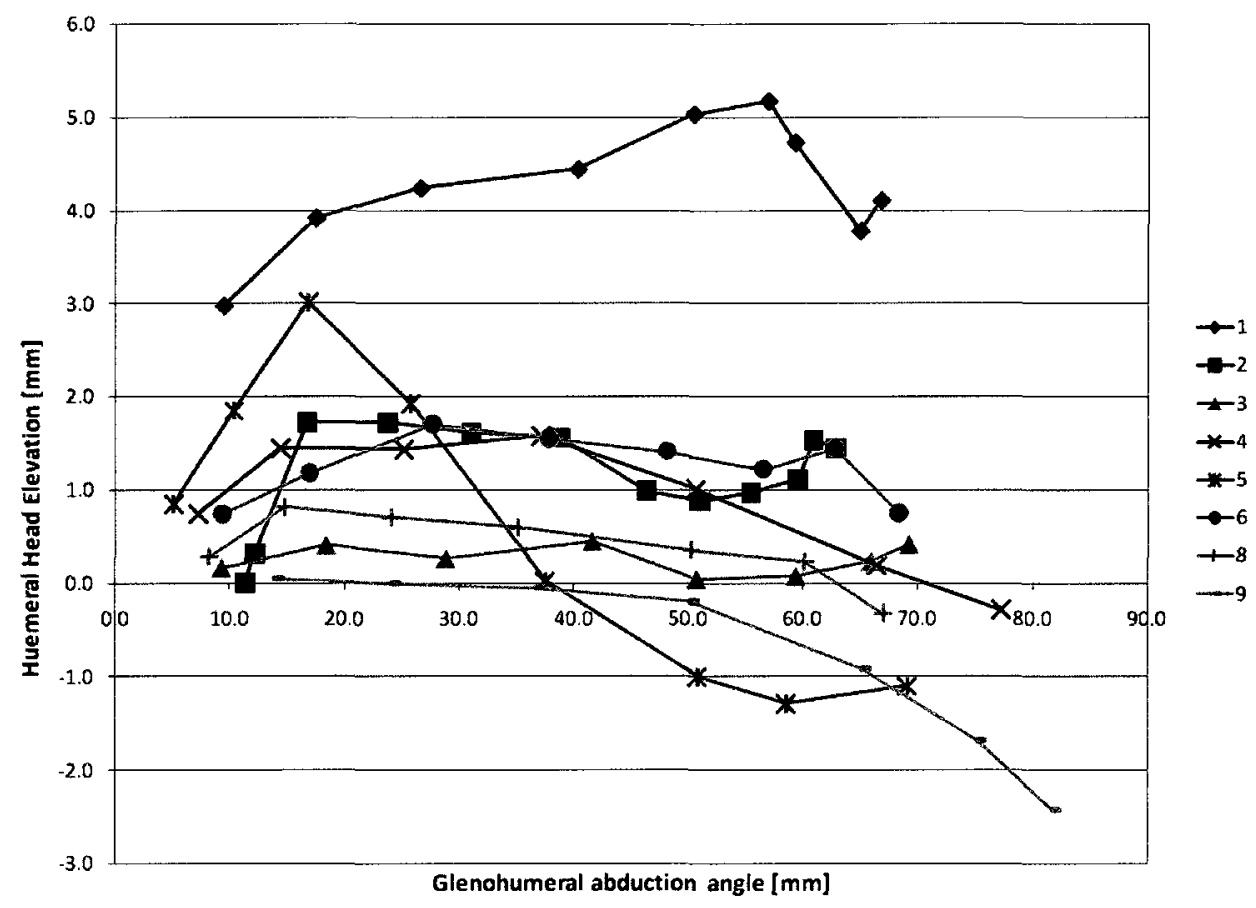

Figure 28: Humeral elevation (mm) versus glenohumeral abduction angle (deg) for each specimen (pre-test data).

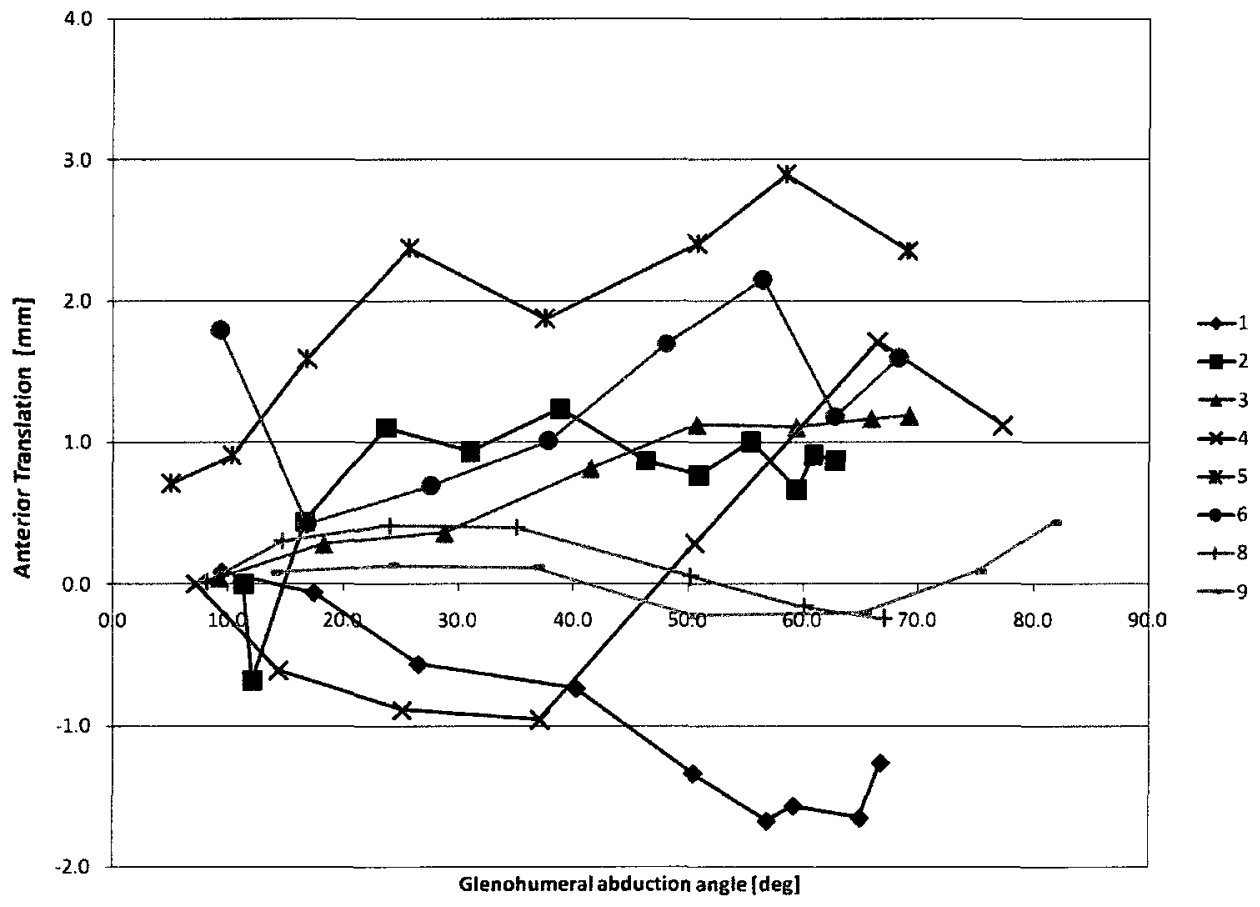

Figure 29: Anterior translation (mm) versus glenohumeral abduction angle (deg) for each specimen (pre-test data). 
Glenohumeral kinematic results at the peak pressure position are included in Table 5. Operative tightening of the posterior capsule did not result in increased superior or anterior translation. A statistically significant difference between plications was noted in external rotation $(\mathrm{p}=0.009)$. A $1 \mathrm{~cm}$ plication resulted in a moderate but significant shift in internal rotation at the position of peak pressure in comparison with a $2 \mathrm{~cm}$ plication $(\mathrm{p}=0.044)$ and approached significance when compared to no plication $(p=0.051)$. No significant difference was noted between treatment groups for all other kinematic outcomes at the position of peak pressure.

Table 5: Glenohumeral kinematics: abduction angle, plane of abduction, external rotation and anterior, superior humeral head translation averaged (mean \pm sd) for all specimens at the position of peak pressure throughout abduction cycle. * denotes significant difference in external rotation for 1 $\mathrm{cm}$ plication group compared to no plication and a $2 \mathrm{~cm}$ plication $(p<0.05)$.

\begin{tabular}{cccccc}
\hline $\begin{array}{c}\text { Posterior } \\
\text { Capsular } \\
\text { Plication }\end{array}$ & \multicolumn{3}{c}{ Euler Angles (deg) } & \multicolumn{2}{c}{ Translation (mm) } \\
\hline \multirow{2}{*}{ None } & Abduction & Plane & Rotation & Superior & Anterior \\
\hline \multirow{2}{*}{$1 \mathrm{~cm}$} & 52.8 & -4.8 & 23.2 & 0.4 & 0.8 \\
& $(8.6)$ & $(9.6)$ & $(11.4)$ & $(2.2)$ & $(1.5)$ \\
\hline \multirow{2}{*}{$2 \mathrm{~cm}$} & 52.1 & -5.2 & $21.7 *$ & 0.6 & 0.5 \\
& $(8.4)$ & $(10.2)$ & $(11.3)$ & $(2.0)$ & $(1.1)$ \\
\hline & 52.1 & -5.4 & 24.1 & 0.4 & 0.4 \\
& $(8.8)$ & $(9.7)$ & $(11.7)$ & $(2.0)$ & $(1.5)$ \\
\hline
\end{tabular}




\section{Chapter 6: Discussion}

\subsection{Shoulder Simulator}

During the pilot specimens $(n=6)$, several design modifications to the shoulder simulator were completed in order to address certain limitations of previous designs. The most important design change was the addition of the deltoid loading pulley assembly. Initially, the intent was to screw an eyelet on the upper surface of the acromion to redirect the cable to a large pulley (Figure 30 ) as described by Wuelker et al. ${ }^{32}$

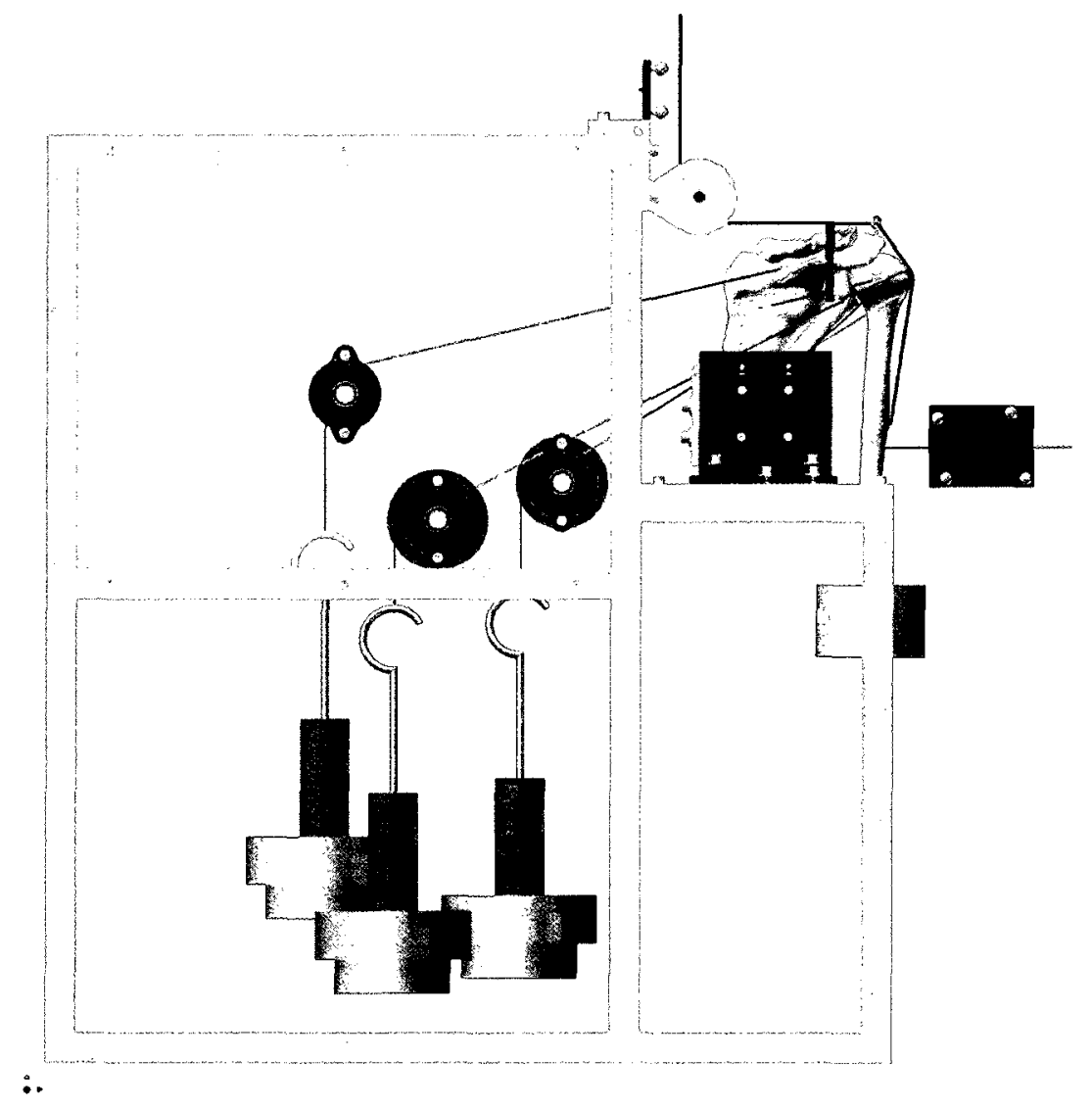

Figure 30: Initially deltoid cable was to be redirected with eyelet attached to acromion. 
With the eyelet design, the deltoid cable would come into contact with the pressure sensor creating artefacts. In addition, it was very difficult to keep the eyelet secured to the thin acromion. The deltoid loading assembly (Figure 14, p.30) reduces friction, keeps the cable at a distance from the sensor, maintains a constant physiological moment arm and provides adjustment to align the cable in the scapular plane.

Similarly, eye bolts were to be used on the scapula near the medial border to maintain the origin of pull of the subscapularis and combined infraspinatus and teres minor muscles. The scapula is relatively thin in this area and it was difficult to achieve good fixation. The eye bolts may have contributed to scapula fracture in one specimen (5). Adjustable eyebolts attached to the potting cup were added to avoid drilling in the scapula (Figure 17, p.33).

Finally, two fracture fixation plates were added to the medial and lateral borders of the scapula to prevent further fractures of the scapula during testing (Figure 31). 


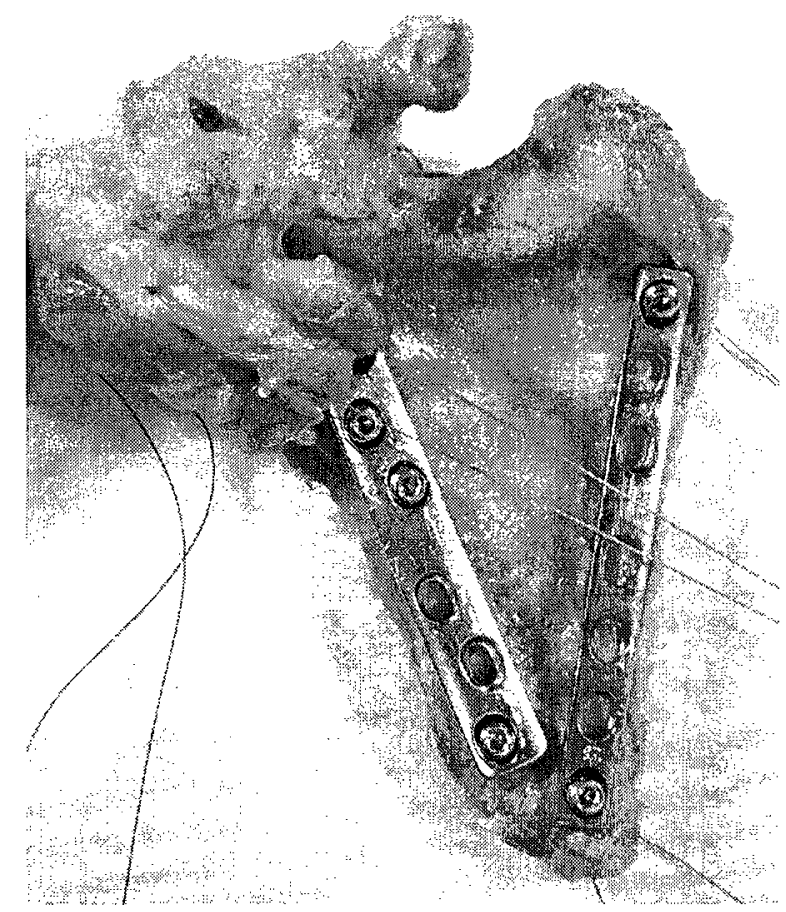

Figure 31: $4.5 \mathrm{~mm}$ fracture fixation plates secured to the medial and lateral borders of the scapula prior to potting.

Limitations of the shoulder simulator must be acknowledged. Scapular abduction was completed in discrete static positions rather than simulating continuous active abduction. To minimize possible effects of discrete measurements on group comparisons, smaller deltoid increments were applied above and below the position of peak pressure as determined during the preliminary trials.

Our shoulder simulator did not include scapular rotation however forces at the glenohumeral joint depend more on the horizontal distance between the center of mass of the arm and the center of rotation of the humerus than on the rotational position of the scapula. ${ }^{12}$ Excluding scapular rotation also changes the relationship between the humeral head and the undersurface of the acromion which may alter the subacromial space and subsequent subacromial pressure. 
Pectoralis major and latissimus dorsi were not included in the simulation. Both muscles are humeral head depressors and their omission results in increased subacromial pressure compared to the physiological situation. Previous shoulder simulators ${ }^{33,53}$ including both studies that measured subacromial pressure ${ }^{2,12}$ did not include scapular rotation or pectoralis major and latissimus dorsi. The long head of the biceps can also act as a humeral head depressor but it was not included in our simulation because biceps activation is minimal during shoulder motion..$^{54,55}$

The muscle force ratio was held constant throughout the range of motion. Various ratios have been utilized from constant to varying based on PCSA, EMG or both. Methodology, patient variability and inherent technical difficulties in determining muscle force ratios from EMG signals probably explain most of the observed differences. In our study, we used the mean muscle force ratios from published simulators ${ }^{2,32-34}$ to represent the most likely average scenario. Functional adaptation due to tightening of the capsule or muscle contraction was not included.

The anterior and posterior portions of the deltoid which mainly affect flexion and extension were also not included in our model. The net vector of the combined anterior, posterior and middle deltoids was directed in the scapular plane. With the rotator cuff muscles balanced such a directed force would result in abduction of the humerus in the scapular plane. 


\subsection{Subacromial Contact Pressure}

Subacromial contact pressure has been previously used as an outcome measure of SI. $^{2,12,13,31,41,56}$ In general, peak pressure as a function of glenohumeral abduction resulted in an inverted u-shaped curve (Figure 23, p.50). Specimen one did not show this relationship but it was believed that an abnormal bump near the greater tubercle contributed to this increase. For this specimen, the peak pressure position was defined at 40 degrees of glenohumeral abduction (Figure 23, p.50). Specimen 2 was excluded from the study because the subacromial pressure exceeded the saturation pressure of the sensor. Specimen 10 (not shown in Figure 23) was also excluded from the study because no contact pressure between the supraspinatus and undersurface of the acromion was measured.

Peak pressure in the no plication group (Figure 25, p. 51) had a mean of $345 \mathrm{kPa}$ (range 93 to $567 \mathrm{kPa}$ ) and occurred on average at 53 degrees of glenohumeral abduction (Table 5, p.56) which corresponds to 78 degrees of clinical abduction when accounting for scapulothoracic motion. ${ }^{29}$ The peak subacromial pressures observed in our study agree with previous clinical and in-vitro studies in relationship to abduction angle $2,12,13$ and fall within the range of reported pressures from in-vitro simulator studies. ${ }^{2,12}$ Werner et al. ${ }^{13}$ measured subacromial pressures in vivo during active abduction in 11 healthy patients with an arterial catheter (1 $\mathrm{mm}$ diameter) inserted into the subacromial bursa. They measured pressure at 30,60, 90 degrees and maximum abduction. Subacromial pressure peaked at 90 degrees of abduction and decreased at maximum abduction. Wuelker et al. ${ }^{31}$ measured subacromial pressure in a cadaver model during simulated 
active abduction in the scapular plane. Peak pressure averaged $566 \mathrm{kPa}$ (range 174 to $1280 \mathrm{kPa}$ ) at a mean glenohumeral abduction angle of 68 degrees. Similarly, Payne et al. ${ }^{2}$ measured subacromial pressure in a cadaver shoulder simulating active abduction in the scapular plane. Peak pressure occurred under the anterolateral aspect of the acromion at 48 degrees of glenohumeral abduction.

Peak subacromial contact pressure was measured with $10 \mathrm{~N}$ deltoid increments around the position of peak pressure as determined during the pre-test trials. Plots of peak subacromial contact pressure versus glenohumeral abduction angle for each specimen and all levels of plication are shown in Appendix B, p.82-85. In 6 of 8 specimens, the peak pressure is contained within the tested range. Specimen 5 and specimen 8 do not include the peak. The position of peak pressure did sometimes vary between pre-test trials which increased uncertainty in the estimate of this position. The scapula fractured during testing of specimen 5. The specimen was re-potted and re-tested but due to time constraints, one trial was completed for both the pre-test and for each condition. For specimen 8, no problems were encountered during testing. For both specimen 5 and specimen 8 , the peak pressure in the measured range was reported as the peak subacromial pressure.

The measurement accuracy of Tekscan can be affected by shear forces and material compliance of the mating surfaces. The effect of shear stresses in the subacromial space during the simulation is unknown but due to the static nature of the measurements, shear stresses were kept to a minimum. With respect to material compliance, subacromial contact occurs between the rotator cuff and the undersurface of the acromion. The sensor was calibrated using a synthetic rubber to best mimic this 
condition. Both shear stresses and material compliance may have an impact on the absolute values of subacromial pressures but will not change relative differences between treatment groups.

The accuracy of the absolute subacromial pressures reported in this study is questionable due to the limitations of the sensor and the omission of humeral head depressors (teres major, latissimus dorsi) as well as scapular rotation in the shoulder simulator. However, we are interested in relative differences between plication levels to answer the study question. The Tekscan pressure measurement system has shown excellent repeatability and given its minimal thickness represents an excellent choice to measure relative differences between treatments. 


\subsection{Glenohumeral Kinematics}

During the pre-test, specimens were elevated to an average glenohumeral abduction angle of 69.9 degrees. The scapula was potted with its medial border vertical to the ground similar to other simulators. ${ }^{2,12}$ Our defined coordinate system is aligned in the y-direction with the spinae scapulae (TS) and the inferior angle (AI) as illustrated in Figure 19, p.37. The vector from TS to AI is inclined in our study specimens on average 8.1 degrees from vertical. Thus, the humerus was abducted to a mean of 78 degrees of glenohumeral abduction (or 117 of shoulder abduction) with respect to vertical.

The aim of the shoulder simulator was to elevate the humerus in the scapular plane with neutral rotation. In our defined coordinate system, neutral rotation consisted of 30 degrees of external rotation due to the angle between the frontal and scapular planes. Figure 26 (p.54) illustrates axial rotation of the humerus as a function of glenohumeral abduction. Due to the nature of the Euler definition, axial rotations for abduction angles less than 20 degrees are highly affected by offsets in the plane of elevation. ${ }^{51}$ For glenohumeral abduction angles greater than 20 degrees, 6 of 8 specimens resulted in axial rotations within 15 degrees of neutral (Figure 26). Exceptions are found with specimens 2 and 6. Specimen 2 was excluded from the study due to saturation of the sensor. Thus, one specimen (6) was internally rotated by approximately 30 degrees. The reason for the internal rotation is unknown but may be due to anatomical differences. Figure 27 (p.54) shows the plane of elevation as a function of the glenohumeral abduction angle. For glenohumeral abduction angles greater than 20 degrees, all specimens resulted in planes of elevation within 20 degrees of the frontal plane. 
In the normal shoulder, superior translation of the humeral head with active arm abduction is thought to be minimal due to the depressor effect of the rotator cuff. ${ }^{42}$ Our findings during the pre-test trials support this observation as peak superior translation was $1.8 \pm 1.6 \mathrm{~mm}$ (Figure 28, p.55). Peak superior translation did not coincide with the position of peak pressure. In general, peak superior translation occurred earlier (20-30 degrees of glenohumeral abduction) while peak pressure occurred between 40-60 degrees (Figure 23). During the early abduction phase, the deltoid force is essentially vertical which results in greater humeral head elevation. As the humerus is further elevated, the vertical component of the deltoid force is balanced by the increased rotator cuff forces which stabilize the humeral head. As the humerus continues to rotate, the greater tubercle comes into closer proximity with the undersurface of the acromion (Figure 32) leading to increased contact pressure.

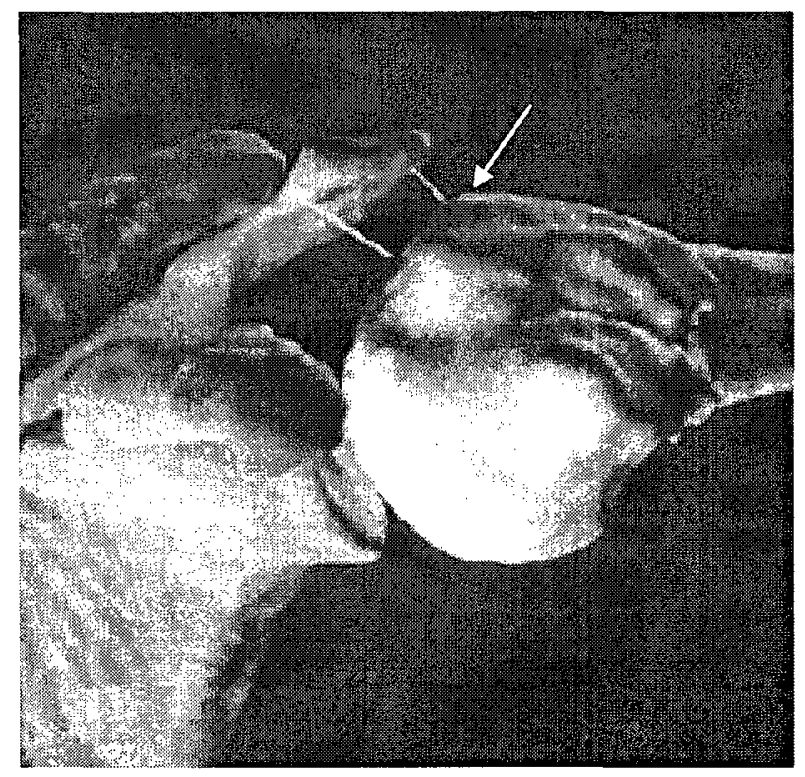

Figure 32: Greater tubercle comes into close proximity with acromion (arrow points to greater tubercle). ${ }^{57}$ 
Anterior translation as a function of glenohumeral abduction angle is shown in Figure 29, p.55. In general, the trend with increasing abduction angle was increased anterior translation. Our measurements of humeral head translations agree with in-vivo measurements. Hallstrom et al. ${ }^{43}$ measured with radiostereometric analysis humeral head translations in healthy patients during active abduction. They found that peak superior translation was approximately $1.3 \mathrm{~mm}$ and occurred early during the abduction cycle (20 degrees of shoulder abduction). They also found that anterior translation increased with increasing abduction angle to a peak of approximately $2 \mathrm{~mm}$ at 60 degrees of shoulder abduction. 


\subsection{Effect of Posterior Capsular Plication}

Tightness of the posterior capsule and/or posterior shoulder has been shown to alter glenohumeral kinematics ${ }^{7,11}$ and has been suggested to contribute to $\mathrm{SI}^{7,8}$ but scientific evidence demonstrating a direct link between posterior capsular plication and increased subacromial pressure is lacking. Results from the current study suggest that posterior capsular plication as a sole variable does not contribute to increased pressure in the subacromial space during abduction in the scapular plane (Figure 25, p.51). In addition, no statistical difference was noted between plication levels in peak pressure, average pressure, contact force or contact area at the position of peak pressure (Table 4, p.52). There was a trend towards increased pressure with a $1 \mathrm{~cm}$ plication although the difference was not statistically significant. Figure 24 (p.50) shows that in 5 of 8 specimens the peak subacromial contact pressure increased from baseline at $1 \mathrm{~cm}$ and returned to baseline for a $2 \mathrm{~cm}$ plication.

In our study, no statistical difference was noted in either abduction angle or plane of abduction at the position of peak pressure. A significant difference in axial rotation was noted between groups: a $1 \mathrm{~cm}$ plication resulted in slightly more internal rotation (approximately 2 degrees) at the position of peak pressure as compared to both the no plication and $2 \mathrm{~cm}$ plication groups. It is difficult to explain this result as one would expect the same phenomenon to occur with a $2 \mathrm{~cm}$ plication. The plication of the medial versus the lateral side of the capsule (see Figure 20) may explain the observed difference in axial rotation although the mechanism by which this occurs is not clear. Of note, the 1 $\mathrm{cm}$ plication group resulted in slightly higher although not significant subacromial 
contact pressure and force and perhaps the small change in axial rotation is secondary to increased contact with the acromion. The clinical significance of two degrees of internal rotation is questionable.

Patients with SI have demonstrated altered glenohumeral kinematics. Altered glenohumeral kinematics during arm elevation, particularly increased superior or anterior humeral head translation, inadequate external rotation and decreased normal scapular upward rotation may result in decreased subacromial space and increased pressure. ${ }^{43}$ Deutsch et al. ${ }^{44}$ and Hallström et al. ${ }^{43}$ both showed significant superior translation during active abduction in patients with SI as compared to healthy controls. Ludewig et al. ${ }^{8}$ on the other hand measured small but significantly greater anterior translation during active abduction in patients with SI but no difference in superior translation. Lin et al." compared glenohumeral kinematics of patients with tight anterior and tight posterior shoulders. They observed increased anterior and superior translation in patients with tight posterior shoulders during abduction in the scapular plane but the authors did not compare groups with either controls or contralateral shoulders.

Results from our study reveal no significant difference in either glenohumeral kinematics or humeral head translation following posterior capsular plication during simulated abduction in the scapular plane suggesting that another mechanism may be responsible for altered glenohumeral kinematics in patients with a tight posterior shoulder. It is generally believed that a tight posterior shoulder with positive impingement signs originates from a thick and shortened capsule but a recent case report suggests that it may also originate from muscular contractures of the infraspinatus and teres minor. ${ }^{14}$ Our findings indirectly support this hypothesis. 
The main strength of the current study is the ability to isolate the effect of posterior capsular plication on glenohumeral kinematics and subacromial pressure. Results show that posterior capsular plication in isolation does not change subacromial pressure during abduction in the scapular plane. However, the posterior capsule is most stretched at the limits of flexion where capsular tightness may contribute to obligate anterior and superior translation and increased subacromial pressure as suggested by Harryman et al. ${ }^{7}$

Clinically, pain in patients with SI is reproduced during the Hawkins ${ }^{1}$ test and $\mathrm{Neer}^{37}$ sign. The Neer sign consists of forward flexion to approximately 160 degrees with neutral rotation while holding the scapula fixed. During the Hawkins test, the humerus is internally rotated at 90 degrees of forward flexion. Both clinical manoeuvres reproduce pain in forward flexion but in the experience of the surgeon involved in this study pain can also be elicited during abduction in the scapular plane. In the current study, motion was constrained to abduction in the scapular plane for which muscle activities are available and rotator cuff muscles are aligned. A simulator for active shoulder flexion must be developed to fully understand the role of posterior capsular tightness as a factor in causation of SI. 


\section{Chapter 7: Conclusions}

\subsection{Conclusions}

Posterior capsular plication, as a sole variable, did not significantly increase subacromial contact during simulated abduction in the scapular plane. Glenohumeral kinematics was similar between plication levels. No significant differences were observed in the angle or plane of abduction at the position of peak pressure. A statistically significant difference in axial rotation was noted following a $1 \mathrm{~cm}$ plication although the clinical significance of 2 degrees is questionable. Finally, posterior capsular plication did not result in a significant increase in anterior or superior translation at the position of peak pressure. 


\subsection{Summary of Contributions}

A shoulder simulator was designed to abduct the humerus in the scapular plane. The simulator showed excellent angular repeatability in abduction, plane of abduction and axial rotation. The simulator will be used in the Orthopaedic Biomechanics Laboratory for future biomechanical studies of the shoulder. A calibration method for the Tekscan pressure measurement system was developed and a Matlab routine was created to communicate with the Polaris tracking system and provide real-time kinematic data of the shoulder.

The study adds clinically relevant and important information regarding the role of the posterior capsule in shoulder impingement. Plication of the posterior capsule does not lead to increased pressure during abduction in the scapular plane nor does it lead to increased superior or anterior translation of the humeral head. It is generally believed that a tight posterior shoulder with positive impingement signs originates from a thick and shortened capsule but a recent case report suggests that it may also originate from muscular contractures of the infraspinatus and teres minor. ${ }^{14}$ Our findings indirectly support this hypothesis. 


\subsection{Recommendations}

The current shoulder simulator may be improved by: (1) adding servomotors and, (2) increasing physiological accuracy. To increase efficiency during data collection, servomotors may be added to each rotator cuff muscle to obtain continuous data. The addition of servomotors would significantly decrease the time required to complete one abduction cycle from 20-30 minutes to 30 seconds. With servomotors, more interventions and repeated cycles could be completed with one fresh-frozen cadaver. In this study, the effect of posterior plication could be studied throughout the full abduction. The simulator was designed with this upgrade in mind leaving sufficient space at the back to add the servomotors.

To more closely represent physiological loading of the shoulder, scapular rotation and humeral head depressors (latissimus dorsi and teres major) should be added. A servomotor attached to the potting cup could rotate the scapula at a prescribed ratio with respect to glenohumeral abduction angle.

To fully characterize the role in causation of posterior capsular tightness during shoulder impingement, a shoulder simulator capable of active forward flexion with internal rotation is required. Such a simulator represents significant work as the lines of action of the muscles would no longer be aligned with the scapular plane. A simulator capable of active flexion and internal/external rotation has yet to be published. 


\section{References}

1. Hawkins RJ, Kennedy JC. Impingement syndrome in athletes. Am J Sports Med 1980;8(3):151-8.

2. Payne LZ, Deng XH, Craig EV, Torzilli PA, Warren RF. The Combined Dynamic and Static Contributors to Subacromial Impingement. Am J Sports Med 1997;25(6):801-8

3. Bigliani LU, Levine WN. Current Concepts Review: Subacromial Impingement Syndrome. J Bone Joint Surg [Am] 1997;79(12):1854-68.

4. Neer CS, II. Anterior Acromioplasty for the Chronic Impingement Syndrome in the Shoulder. J Bone Joint Surg [Am] 1972;54(1):41-50.

5. Jobe FW, Kvitne RS, Giangarra CE. Shoulder pain in the overhand or throwing athlete. The relationship of anterior instability and rotator cuff impingement. Orthop Rev 1989;18(9):963-75.

6. Kamkar A, Irrgang JJ, Whitney SL. Nonoperative management of secondary shoulder impingement syndrome. J Orthop Sports Phys Ther 1993;17(5):212-24.

7. Harryman DT, Sidles JA, Clark JM, McQuade KJ, Gibb TD, Matsen FA, III. Translation of the humeral head on the glenoid with passive glenohumeral motion. J Bone Joint Surg Am 1990 Oct;72(9):1334-43.

8. Ludewig PM, Cook TM. Translations of the humerus in persons with shoulder impingement symptoms. J Orthop Sports Phys Ther 2002 Jun;32(6):248-59.

9. Ticker JB, Beim GM, Warner JJ. Recognition and treatment of refractory posterior capsular contracture of the shoulder. Arthroscopy 2000 Jan;16(1):27-34. 
10. Werner CML, Nyffeler RW, Jacob HAC, Gerber C. The effect of capsular tightening on humeral head translations. J Orthop Res 2004;22:194-201.

11. Lin JJ, Lim HK, Yang JL. Effect of shoulder tightness on glenohumeral translation, scapular kinematics, and scapulohumeral rhythm in subjects with stiff shoulders. J Orthop Res 2006 May;24(5):1044-51.

12. Wuelker N, Plitz W, Roetman B. Biomechanical data concerning the shoulder impingement syndrome. Clin Orthop Relat Res 1994 Jun;(303):242-9.

13. Werner MLW, Blumenthal S, Curt A, Gerber C. Subacromial pressures in vivo and effects of selective experimental suprascapular nerve block. J Shoulder Elbow Surg 2006;15(3):319-23.

14. Poser A, Casonato O. Posterior glenohumeral stiffness: Capsular or muscular problem? A case report. Man Ther 2008;13(2):165-70.

15. Gregory T, Hansen U, Emery RJ, Augereau B, Amis AA. Developments in shoulder arthroplasty. Proc Inst Mech Eng [H] 2007;221(1):87-96.

16. eOrthopod 2009. Available from URL: http://www.eorthopod.com

17. Pearl L. Proximal humeral anatomy in shoulder arthroplasty: Implications for prosthetic design and surgical technique. J Shoulder Elbow Surg 2005;14(1S):99S-104S

18. Boileau P, Sinnerton RJ, Chuinard C, Walch G. Arthroplasty of the shoulder. J Bone Joint Surg [Br] 2006;88-B:562-75.

19. Williams GR, Abboud JA. Total shoulder arthroplasty: Glenoid component design. J Shoulder Elbow Surg 2005;14(1S):122S-8S. 
20. Della Valle CJ, Rokito AS, Birdzell MG, Zuckerman JD. Biomechanics of the Shoulder. In: Nordin M, Frankel VH, editors. Basic Biomechanics of the Musculoskeletal System. 3rd ed. Baltimore: Lippincott Williams \& Wilkins; 2001. p. 318-39.

21. Walch G, Edwards B, Boulahia A, Boileau P, Mole D, Adeleine P. The influence of glenohumeral prosthetic mismatch on glenoid radiolucent lines. J Bone Joint Surg $[\mathrm{Am}] 2002 ; 84-\mathrm{A}(12): 2186-91$.

22. Karduna AR, Williams GR, Williams JL, Iannoti JP. Glenohumeral joint translations before and after total shoulder arthroplasty. A study in cadavera. J Bone Joint Surg [Am] 1997;79:1166-74.

23. University of Washington Orthopaedics and Sports Medicine 2009. Available from URL: http://www.orthop.washington.edu/uw/rotatorcuff/tabID 3376/ItemID 202/Pa geID_404/Articles/Default.aspx

24. Hess SA. Functional stability of the glenohumeral joint. Man Ther 2000 May;5(2):63-71.

25. 20th U S edition of Gray's Anatomy of the Human Body 2009. Available from URL: http://en.wikipedia.org/wiki/File:Gray326.png

26. A D A M Health Illustrated Encyclopedia 2009. Available from URL: http://adam.about.com/encyclopedia/Maindex.htm

27. Georgia Perimeter College 2009. Available from URL: http://facstaff.gpc.edu/ jaliff/anahumus.htm 
28. Kuechle DK, Newman SR, Itoi E, Niebur GL, Morrey BF, An KN. The relevance of the moment arm of shoulder muscles with respect to axial rotation of the glenohumeral joint in four positions. Clin Biomech 2000;15(5):322-9.

29. Freedman L, Munro RR. Abduction of the arm in the scapular plane: scapular and glenohumeral movements. A roentgenographic study. J Bone Joint Surg [Am] 1966;48(8):1503-10.

30. Wuelker N, Schmotzer H, Thren K, Korell M. Translation of the glenohumeral joint with simulated active elevation. Clin Orthop Relat Res 1994 Dec;(309):193200.

31. Wuelker N, Roetman B, Roessig S. Coracoacromial pressure recordings in a cadaveric model. J Shoulder Elbow Surg 1995 Nov;4(6):462-7.

32. Wuelker N, Wirth CJ, Roetman B. A Dynamic Shoulder Model: Reliability Testing and Muscle Force Study. J Biomech 1995;28(5):489-99.

33. Bono CM, Renard R, Levine RG, Levy AS. Effect of displacement of fractures of the greater tuberosity on the mechanics of the shoulder. J Bone Joint Surg [Br] 2001;83(7):1056-62.

34. Mura N, O'Driscoll SW, Zobitz ME, Heers G, Jenkyn TR, Chou SM, et al. The effect of infraspinatus disruption on glenohumeral torque and superior migration of the humeral head: a biomechanical study. J Shoulder Elbow Surg 2003;12(2):179-84.

35. Burns WC, Whipple TL. Anatomic relationships in the shoulder impingement syndrome. Clin Orthop Relat Res 1993 Sep;(294):96-102. 
36. Getz JD, Recht MP, Piraino DW, Schils JP, Latimer BM, Jellema LM, et al. Acromial morphology: relation to sex, age, symmetry, and subacromial enthesophytes. Radiology 1996 Jun;199(3):737-42.

37. Neer CS. Impingement lesions. Clin Orthop Relat Res 1983 Mar;(173):70-7.

38. Graichen H, Bonel H, Stammberger T, Haubner M, Rohrer H, Englmeier KH, et al. Three-dimensional analysis of the width of the subacromial space in healthy subjects and patients with impingement syndrome. AJR Am J Roentgenol 1999 Apr;172(4):1081-6.

39. Morrison DS, Frogameni AD, Woodworth P. Non-operative treatment of subacromial impingement syndrome. J Bone Joint Surg [Am] 1997;79:732-7.

40. Soyer J, Vaz S, Pries P, Clarac JP. The relationship between clinical outcomes and the amount of arthroscopic acromial resection. Arthroscopy 2003 Jan;19(1):34-9.

41. Nordt WE, III, Garretson RB, III, Plotkin E. The measurement of subacromial contact pressure in patients with impingement syndrome. Arthroscopy 1999 Mar;15(2):121-5.

42. Graichen H, Stammberger T, Bonel H, Englmeier KH, Reiser M, Eckstein F. Glenohumeral translation during active and passive elevation of the shoulder - a 3D open-MRI study. J Biomech 2000;33:609-13.

43. Hallstrom E, Karrholm J. Shoulder kinematics in 25 patients with impingement and 12 controls. Clin Orthop Relat Res 2006 Jul;448:22-7. 
44. Deutsch A, Altchek DW, Schwartz E, Otis JC, Warren RF. Radiologic measurement of superior displacement of the humeral head in the impingement syndrome. J Shoulder Elbow Surg 1996;5(3):186-93.

45. Warner JJ, Micheli LJ, Arslanian LE, Kennedy J, Kennedy R. Patterns of flexibility, laxity, and strength in normal shoulders and shoulders with instability and impingement. Am J Sports Med 1990;18(4):366-75.

46. Tyler TF, Nicholas SJ, Roy T, Gleim GW. Quantification of posterior capsule tightness and motion loss in patients with shoulder impingement. Am J Sports Med 2000 Sep;28(5):668-73.

47. Gerber C, Werner CML, Macy JC, Jacob HAC, Nyffeler RW. Effect of Selective Capsulorrhaphy on the Passive Range of Motion of the Glenohumeral Joint. J Bone Joint Surg [Am] 2003;85(1):48-55.

48. Johnson GR, Spaling D, Nowitke A, Bogduk N. Modelling the muscles of the scapula morphometric and coordinate data and functional implications. J Biomech 1996;29(8):1039-51.

49. Wu G, van der Helm FCT, Veeger HEJ, Makhsous M, Van Roy P, Anglin C, et al. ISB recommendation on definitions of joint coordinate systems of various joints for the reporting of human joint motions - Part II: shoulder, elbow, wrist and hand. J Biomech 2005;38(5):981-92.

50. Meskers CG, van Der Helm FC, Rozendaal LA, Rozing PM. In vivo estimation of the glenohumeral joint rotation center from scapular bony landmarks by linear regression. J Biomech 1998 Jan;31(1):93-6. 
51. Masuda T, Ishida A, Cao L, Morita S. A proposal for a new definition of the axial rotation angle of the shoulder joint. J Electromyogr Kinesiol 2008;18(1):154-9.

52. Kumar VP, Satku SK. Documenting rotation at the glenohumeral joint. A technical note. Acta Orthop Scand 1994;65(4):483-4.

53. Kedgley AE, Mackenzie GA, Ferreira LM, Drosdowech DS, King GJ, Faber KJ, et al. The effect of muscle loading on the kinematics of in vitro glenohumeral abduction. J Biomech 2007;40(13):2953-60.

54. Yamaguchi K, Riew KD, Galatz LM, Syme JA, Neviaser RJ. Biceps Activation During Shoulder Motion. An Electromyographic Analysis. Clin Orthop Relat Res $1997 ; 336: 122-9$.

55. Kido $\mathrm{T}$, Itoi $\mathrm{T}$, Konno N, Sano A, Urayama M, Sato K. Electromyographic activities of the biceps during arm elevation in shoulders with rotator cuff tears. Acta Orthop Scand 1998;69(6):575-9.

56. Hyvonen P, Lantto V, Jalovaara P. Local pressures in the subacromial space. Int Orthop 2003;27(6):373-7.

57. Graichen H, Bonel H, Stammberger T, Englmeier KH, Reiser M, Eckstein F. Subacromial space width changes during abduction and rotation--a 3-D MR imaging study. Surg Radiol Anat 1999;21(1):59-64. 
Appendix 
Appendix A: Randomization Schedule

\begin{tabular}{ccccccccccc}
\hline & \multicolumn{10}{c}{ Specimen Number } \\
Sequence & $\mathbf{1}$ & $\mathbf{2}$ & $\mathbf{3}$ & $\mathbf{4}$ & $\mathbf{5}$ & $\mathbf{6}$ & $\mathbf{7}$ & $\mathbf{8}$ & $\mathbf{9}$ & $\mathbf{1 0}$ \\
\hline $\mathbf{1}$ & 0 & 0 & 2 & 1 & 2 & 0 & 1 & 1 & 2 & 0 \\
$\mathbf{2}$ & 1 & 1 & 1 & 0 & 1 & 1 & 2 & 2 & 1 & 1 \\
\hline
\end{tabular}


Appendix B: Peak Subacromial Pressure versus Abduction Angle

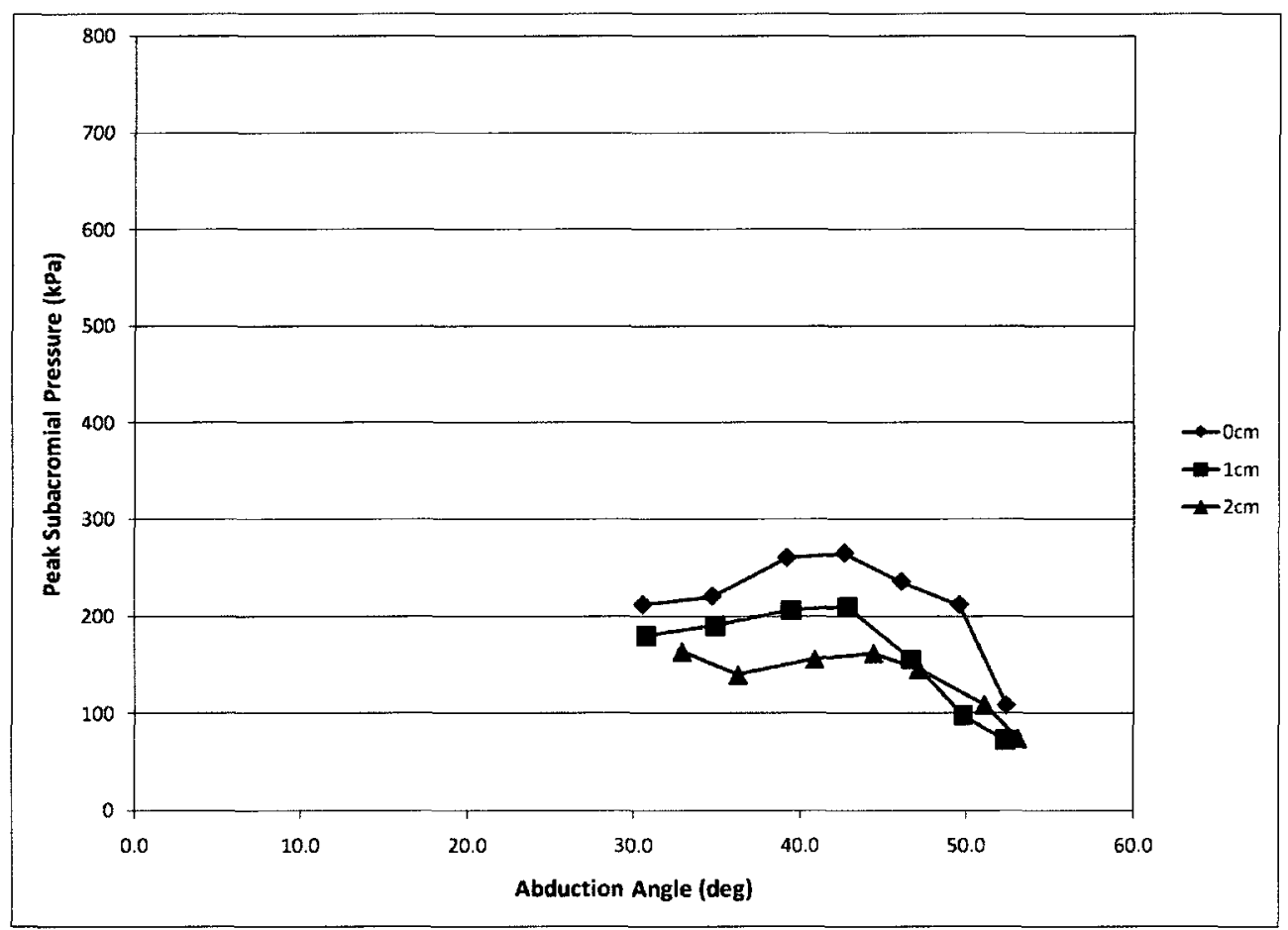

Specimen 1

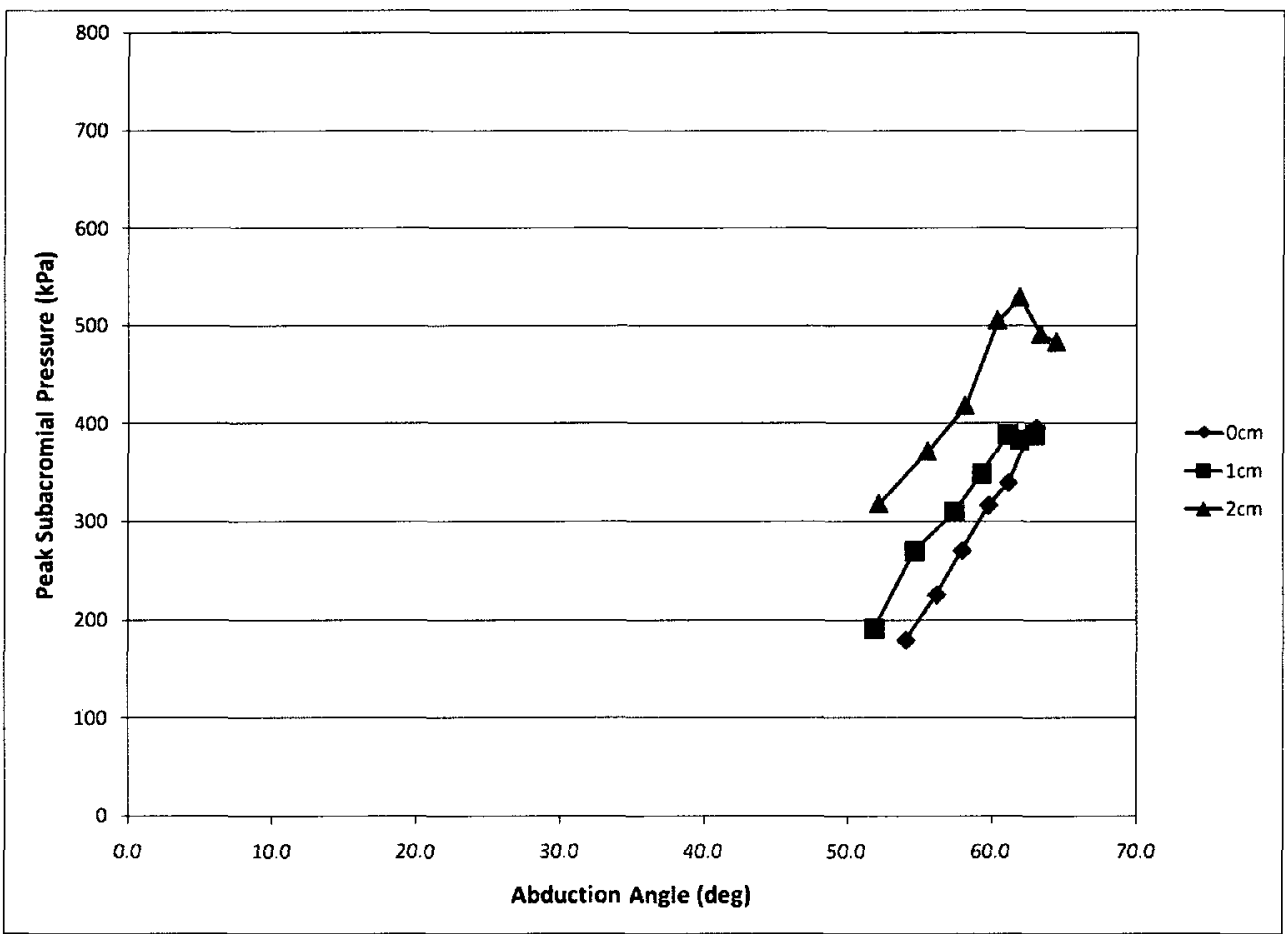

Specimen 3 


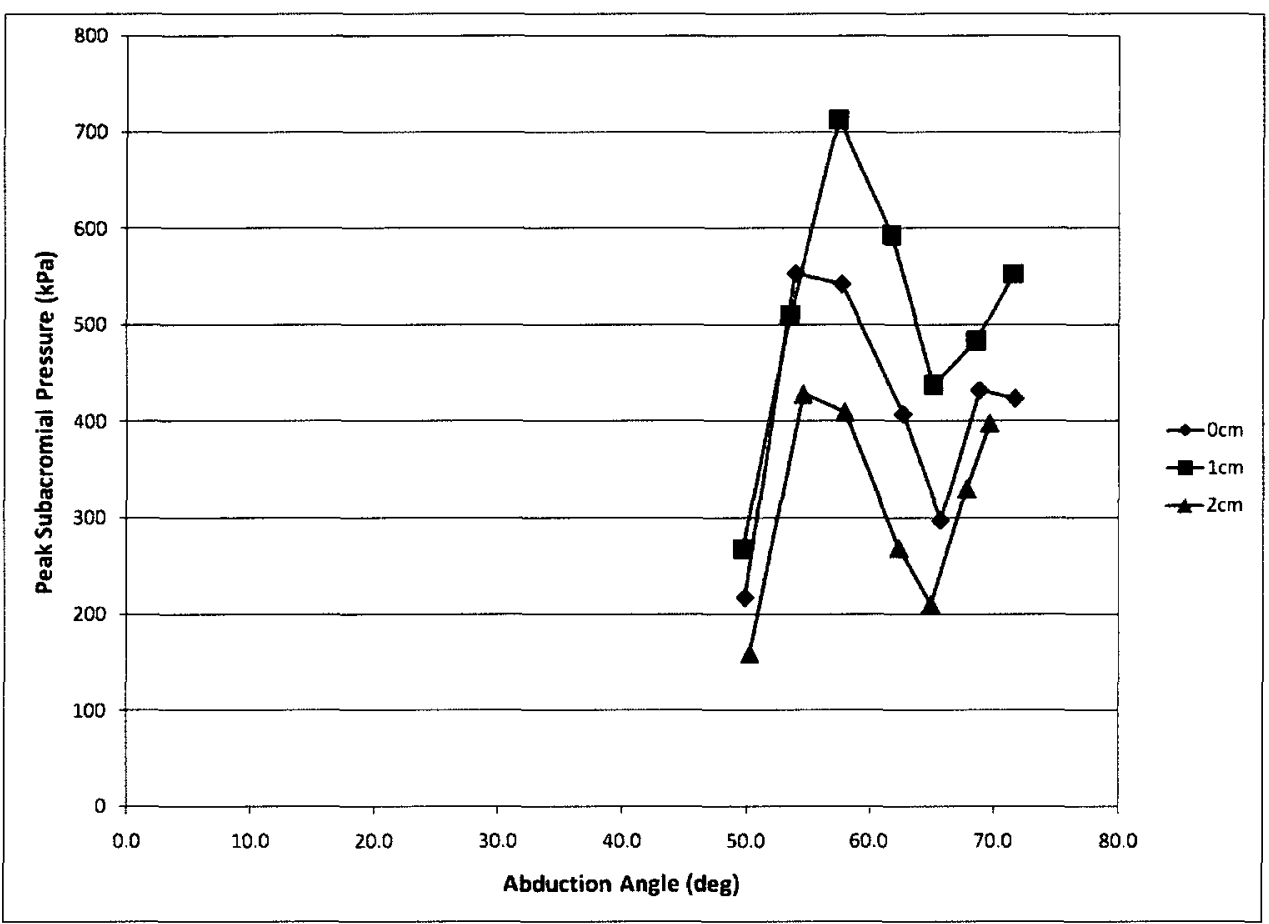

\section{Specimen 4}

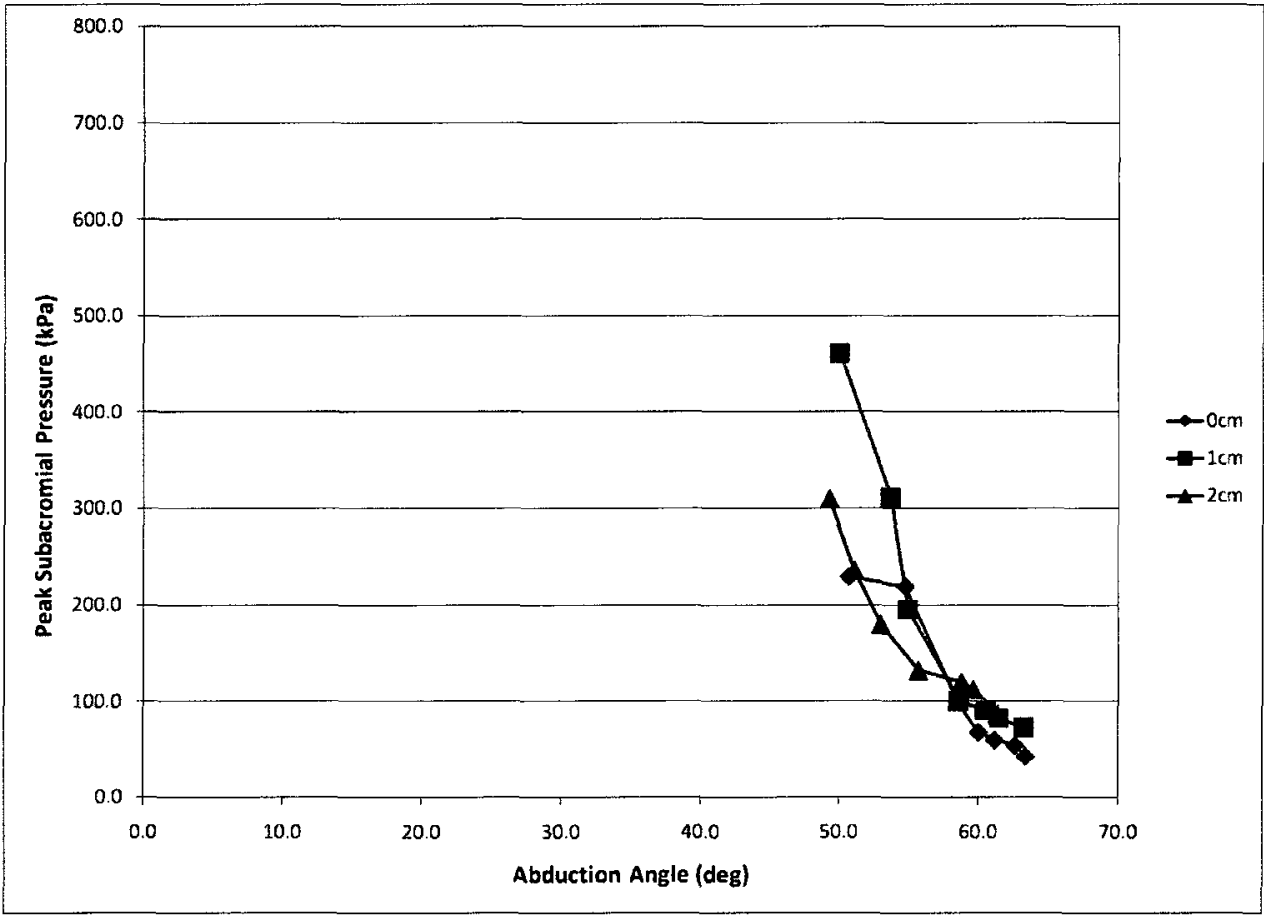

Specimen 5 


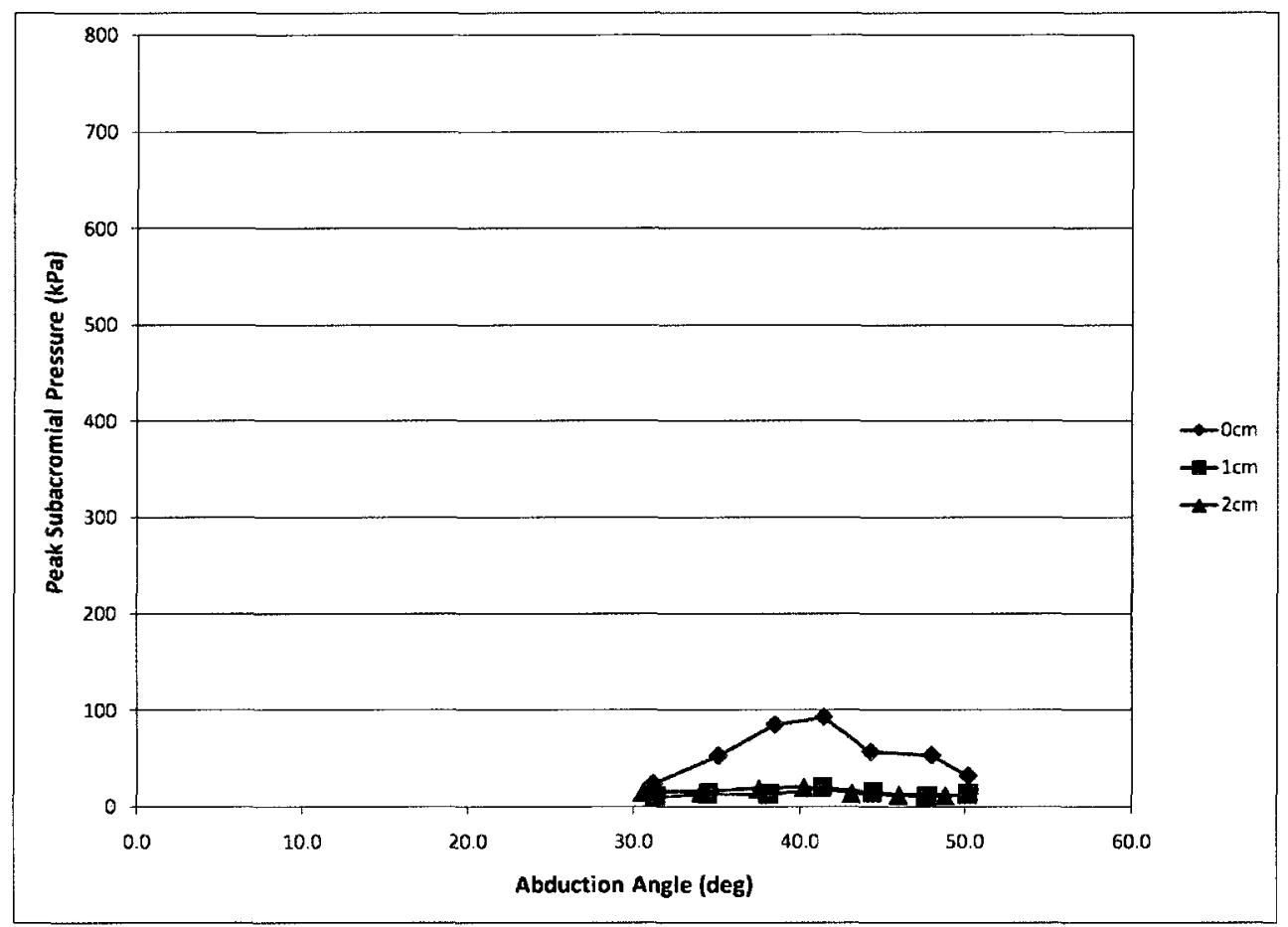

Specimen 6

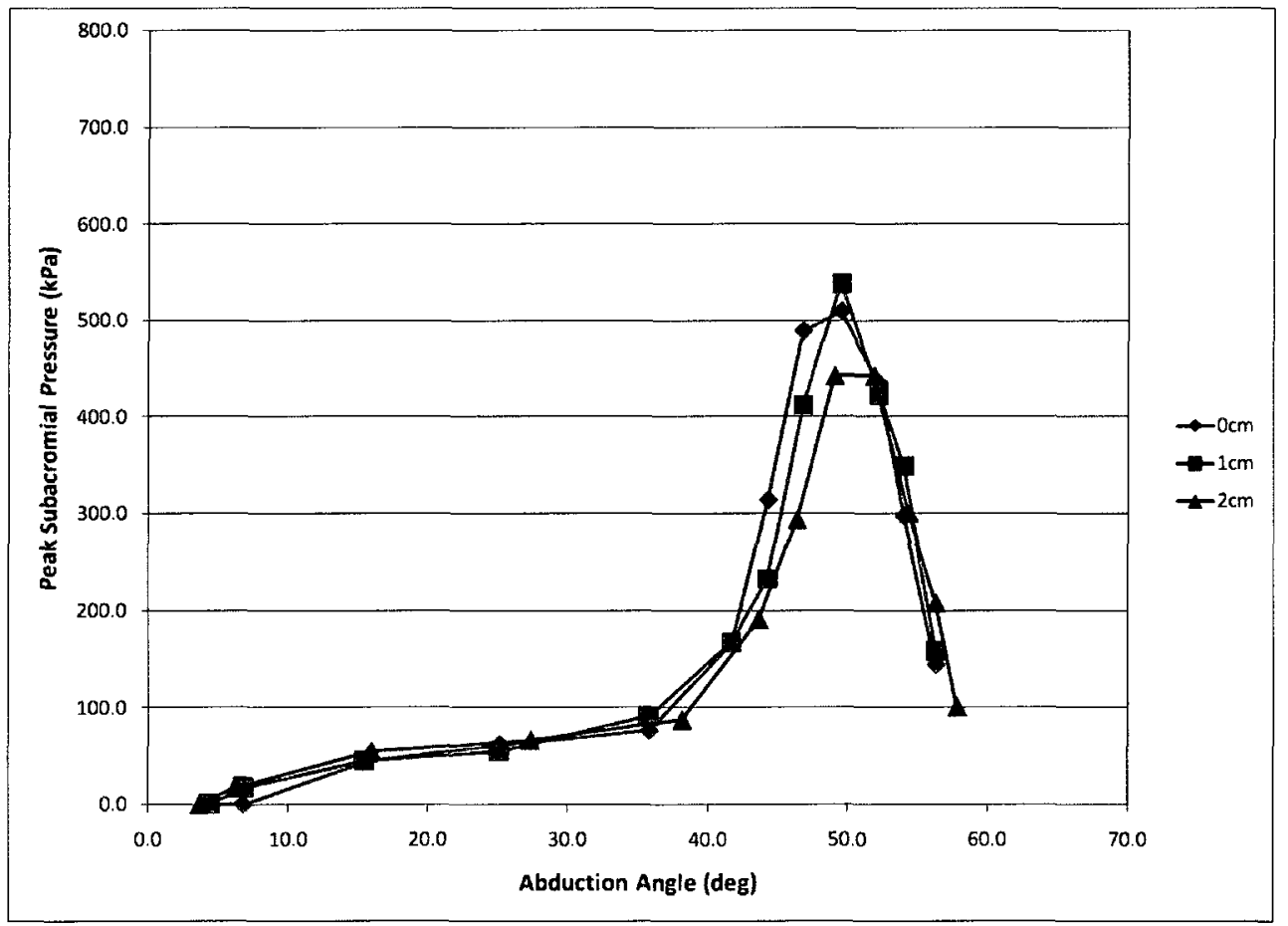

Specimen 7 


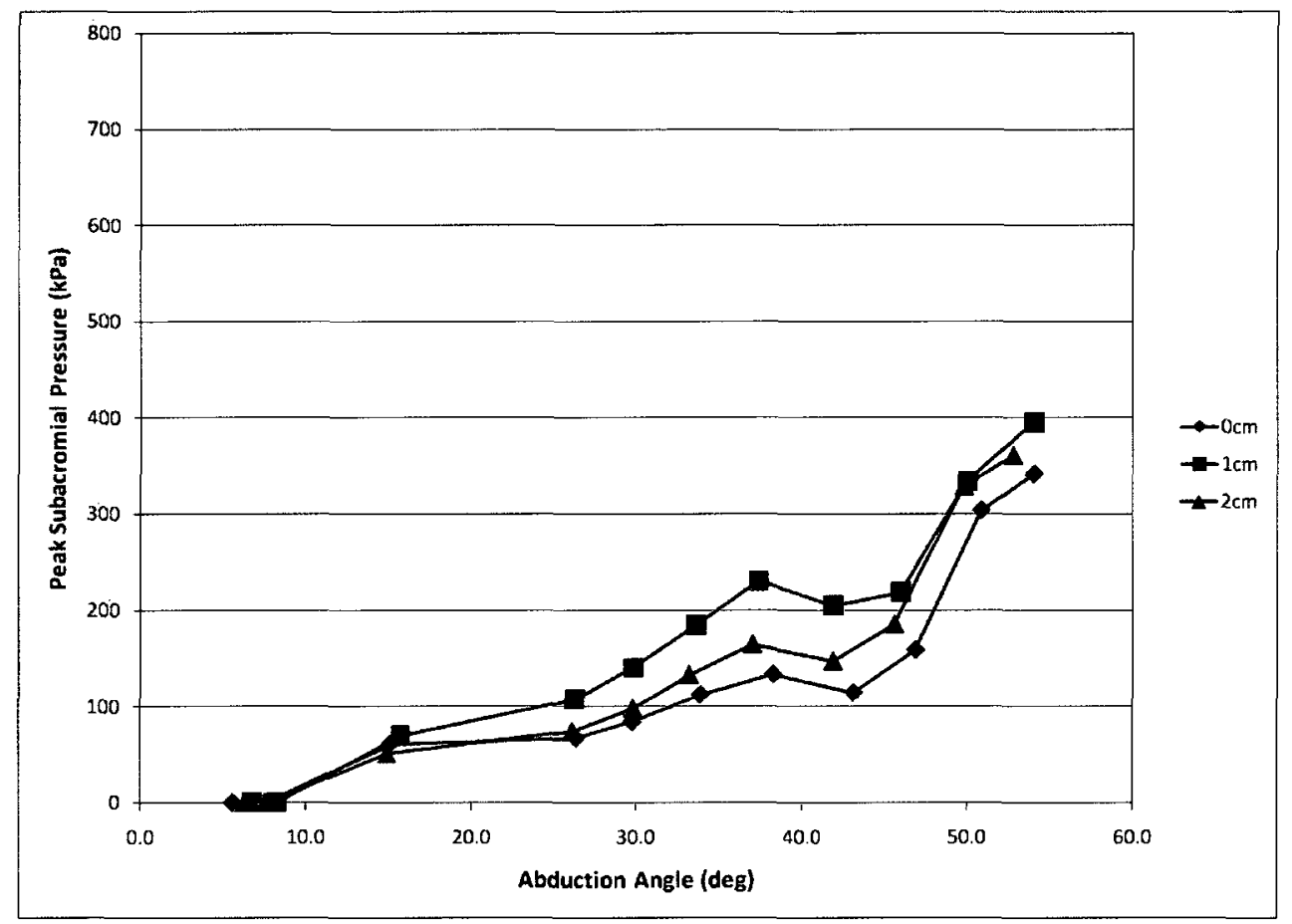

Specimen 8

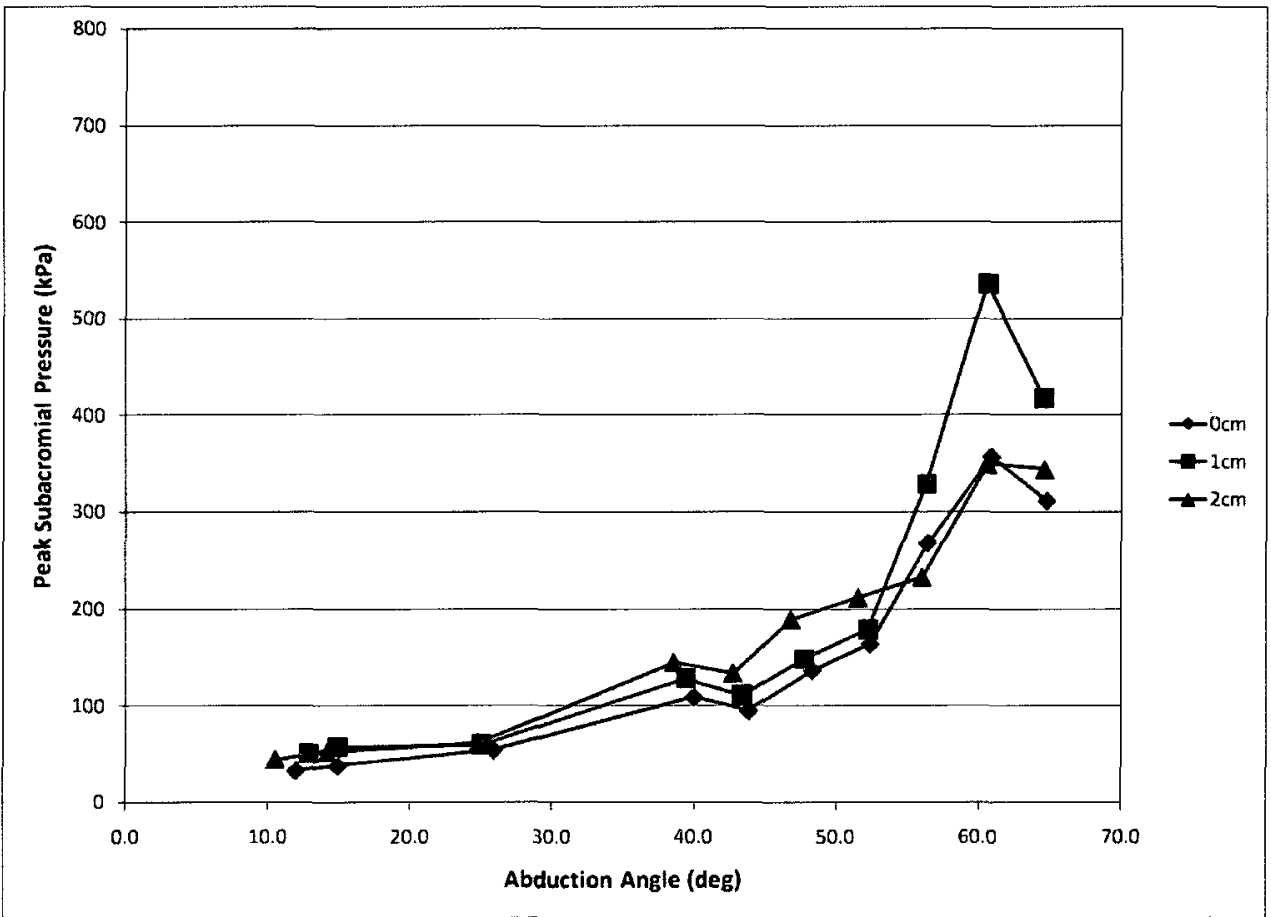

Specimen 9 
Appendix C.1: Shoulder Simulator-Engineering Drawings

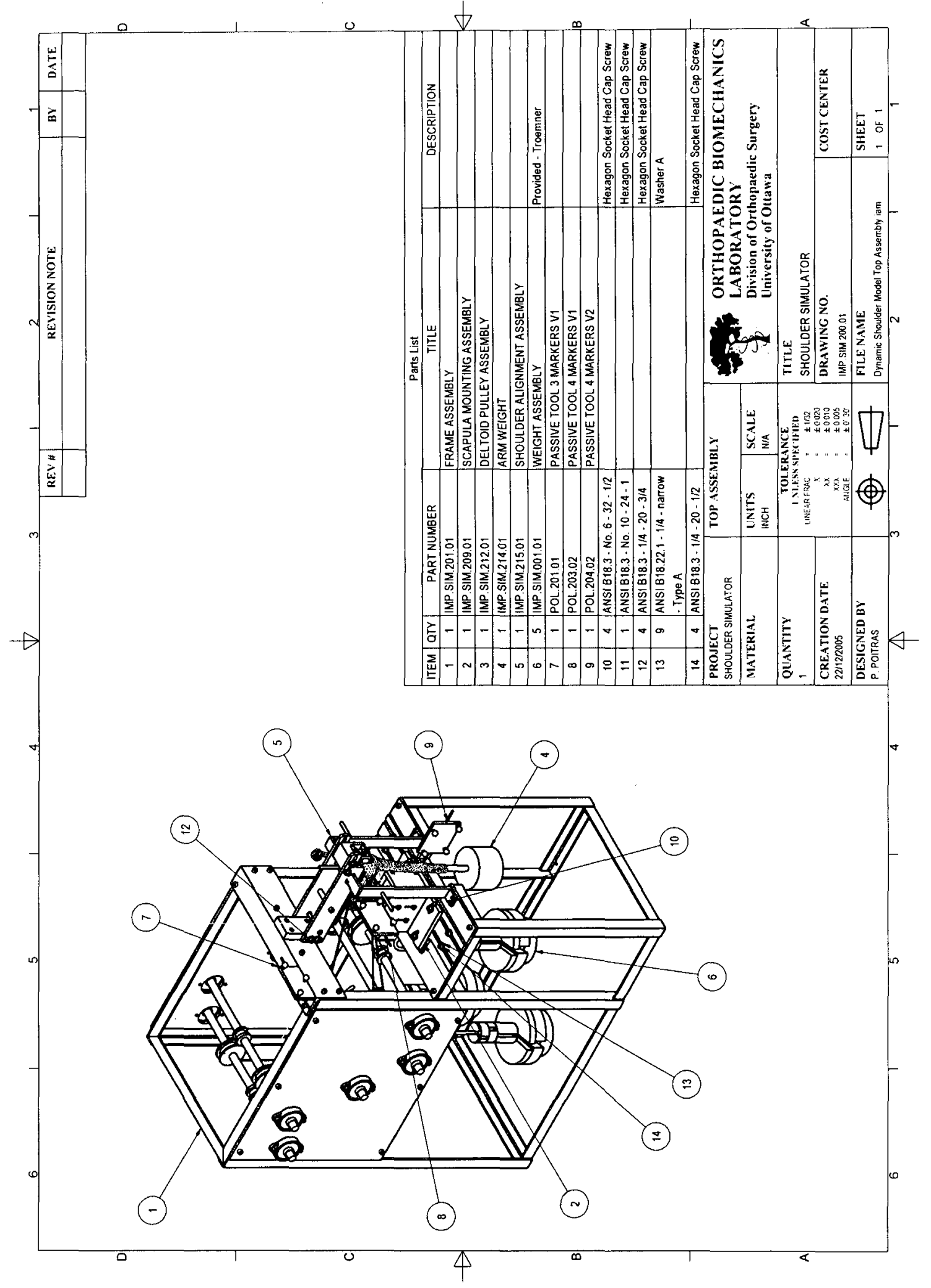




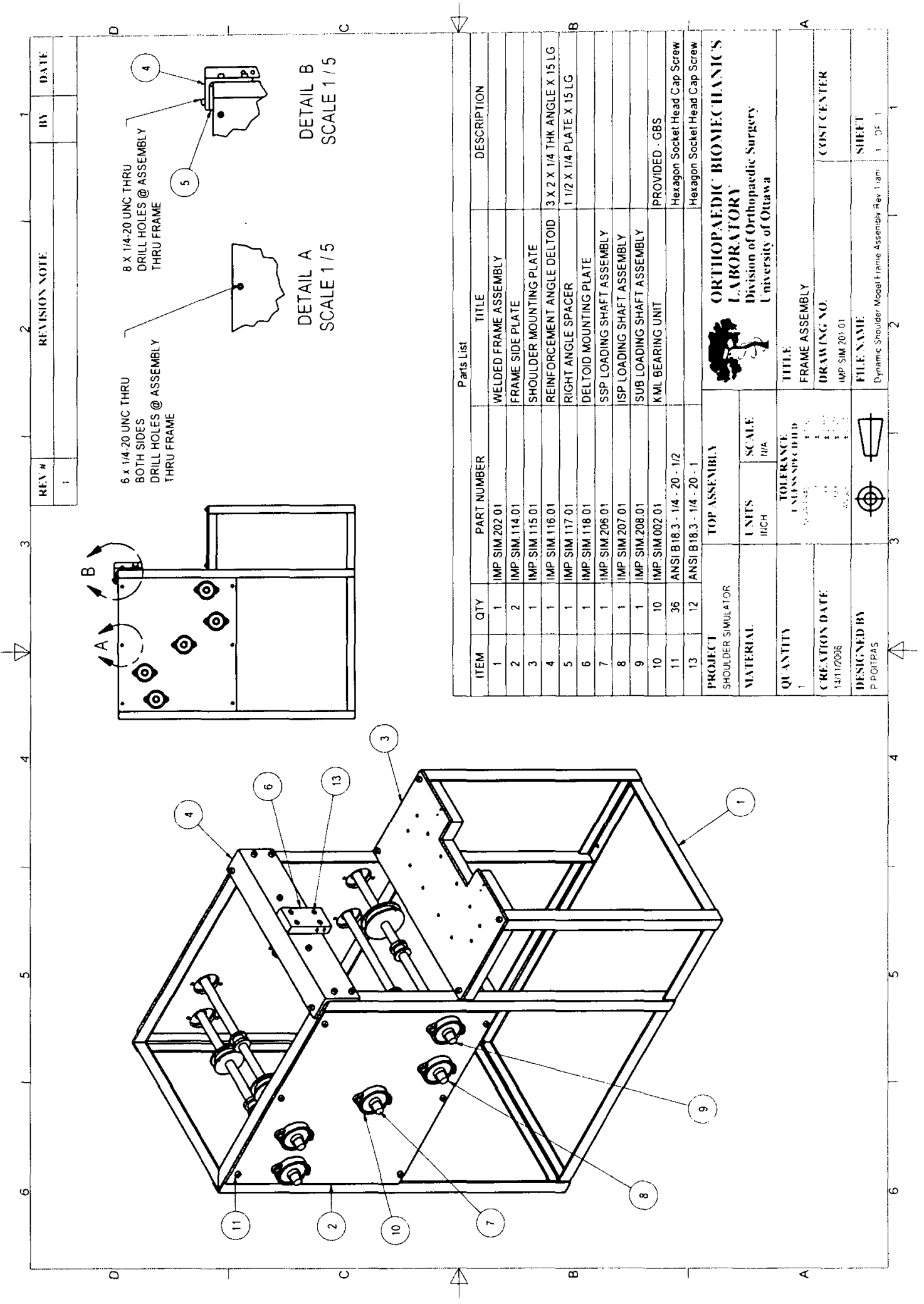




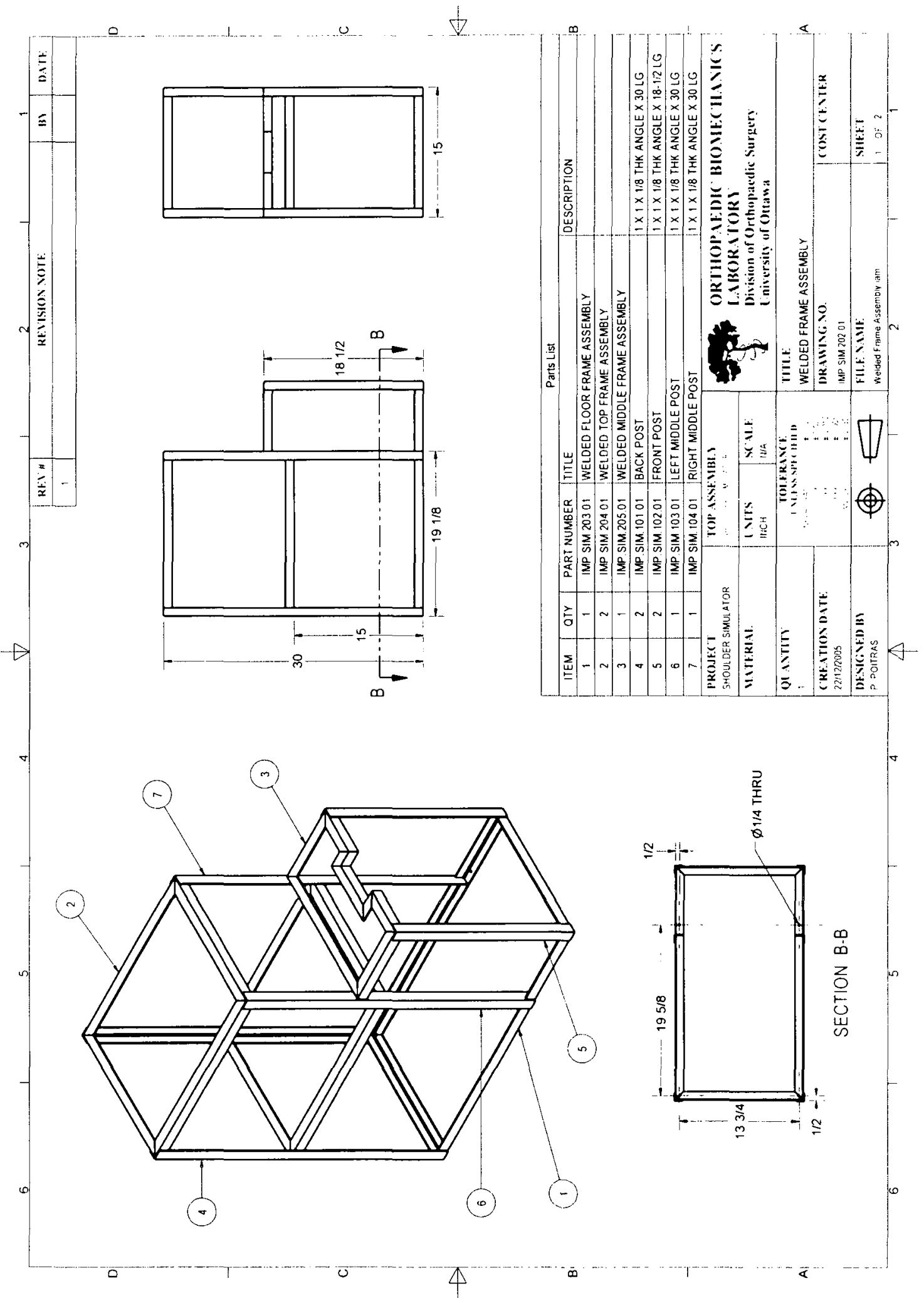




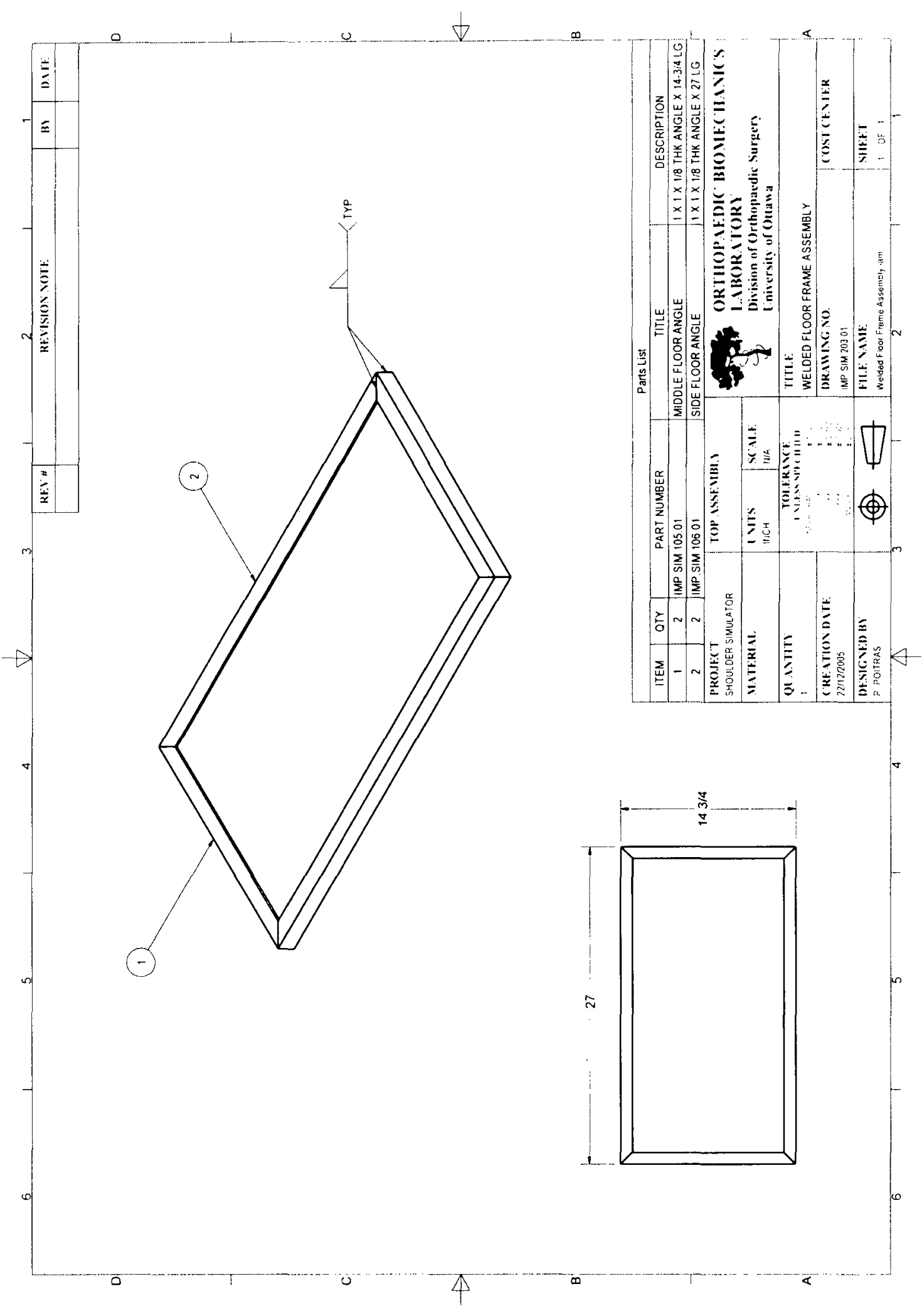




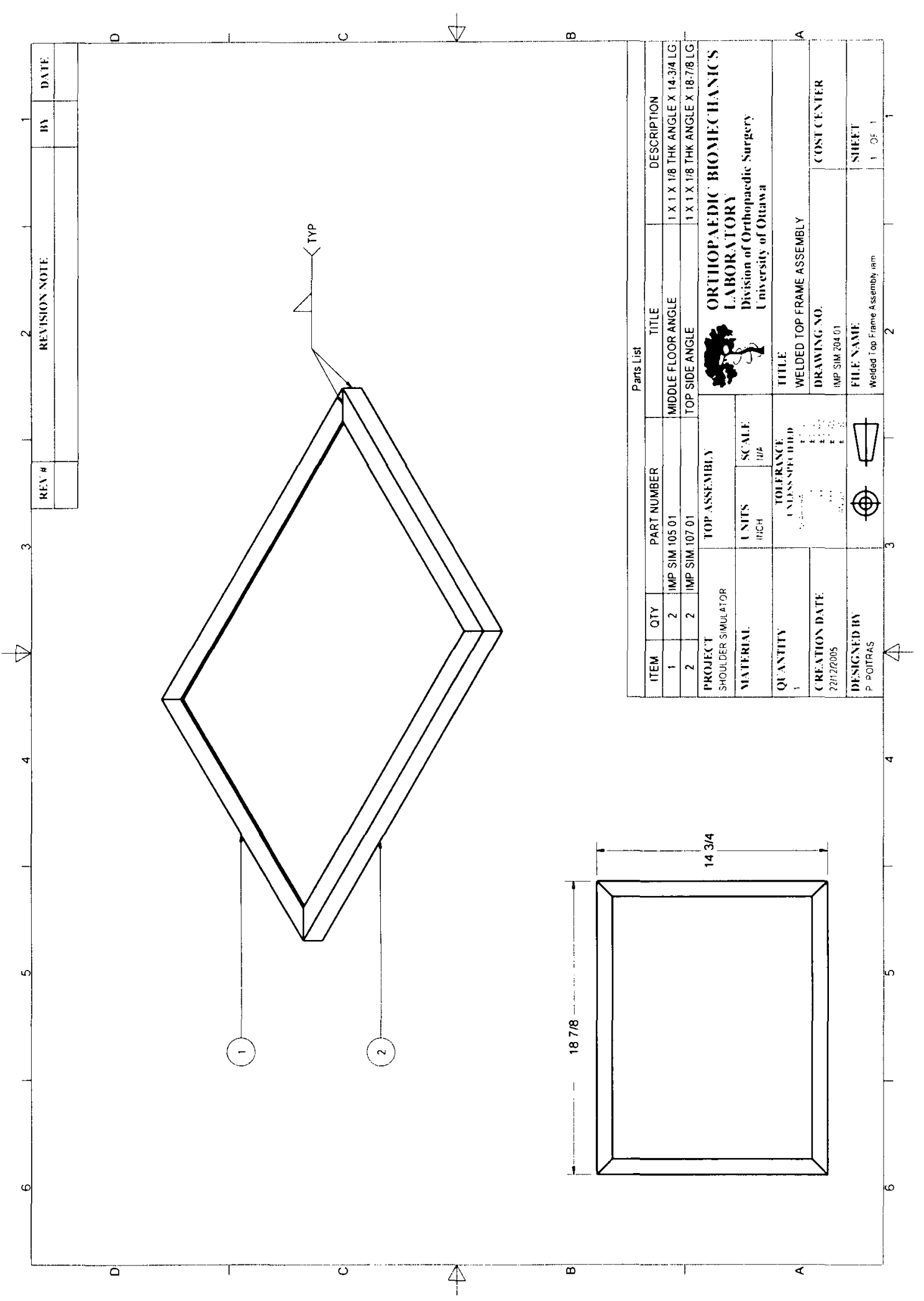




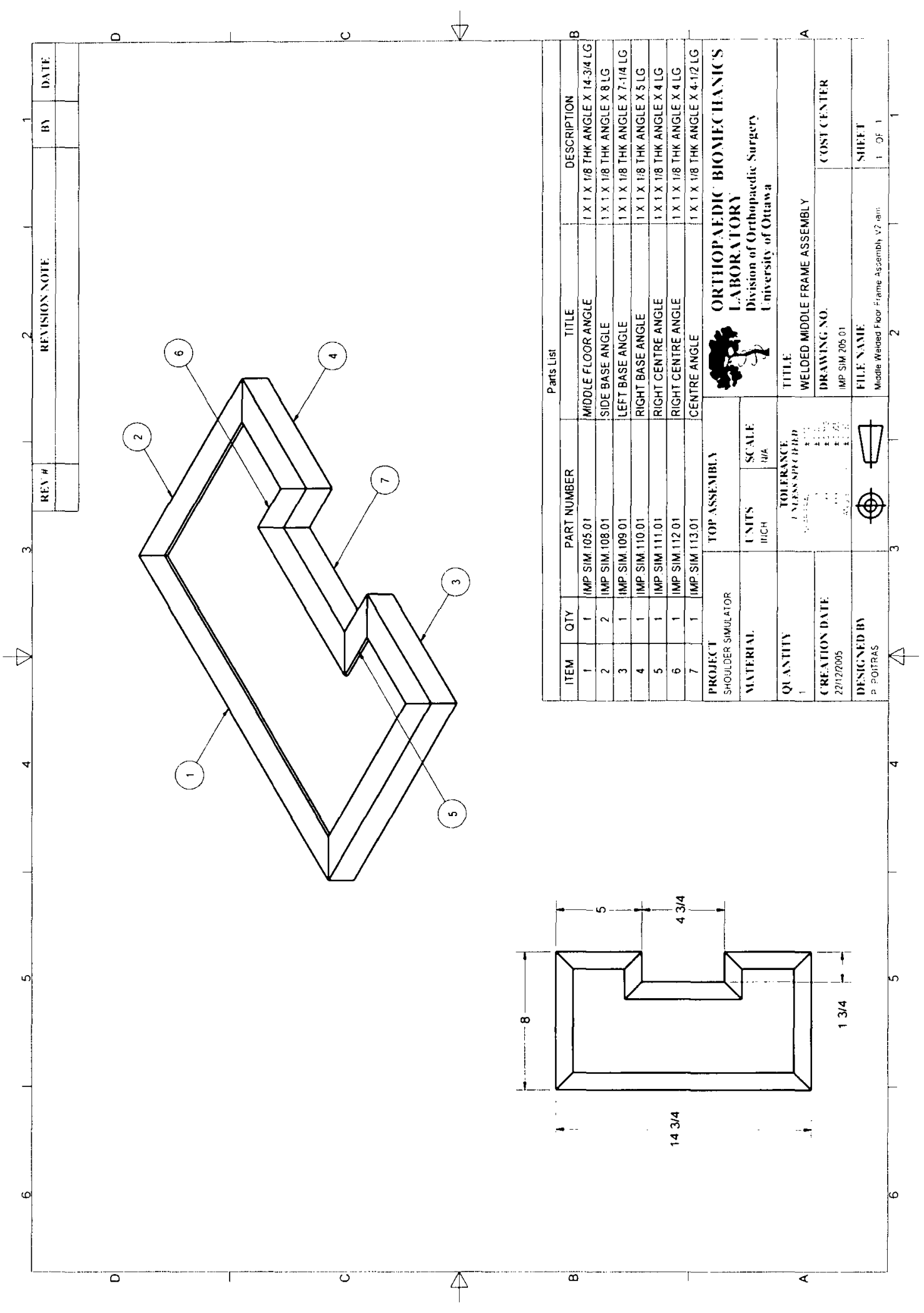




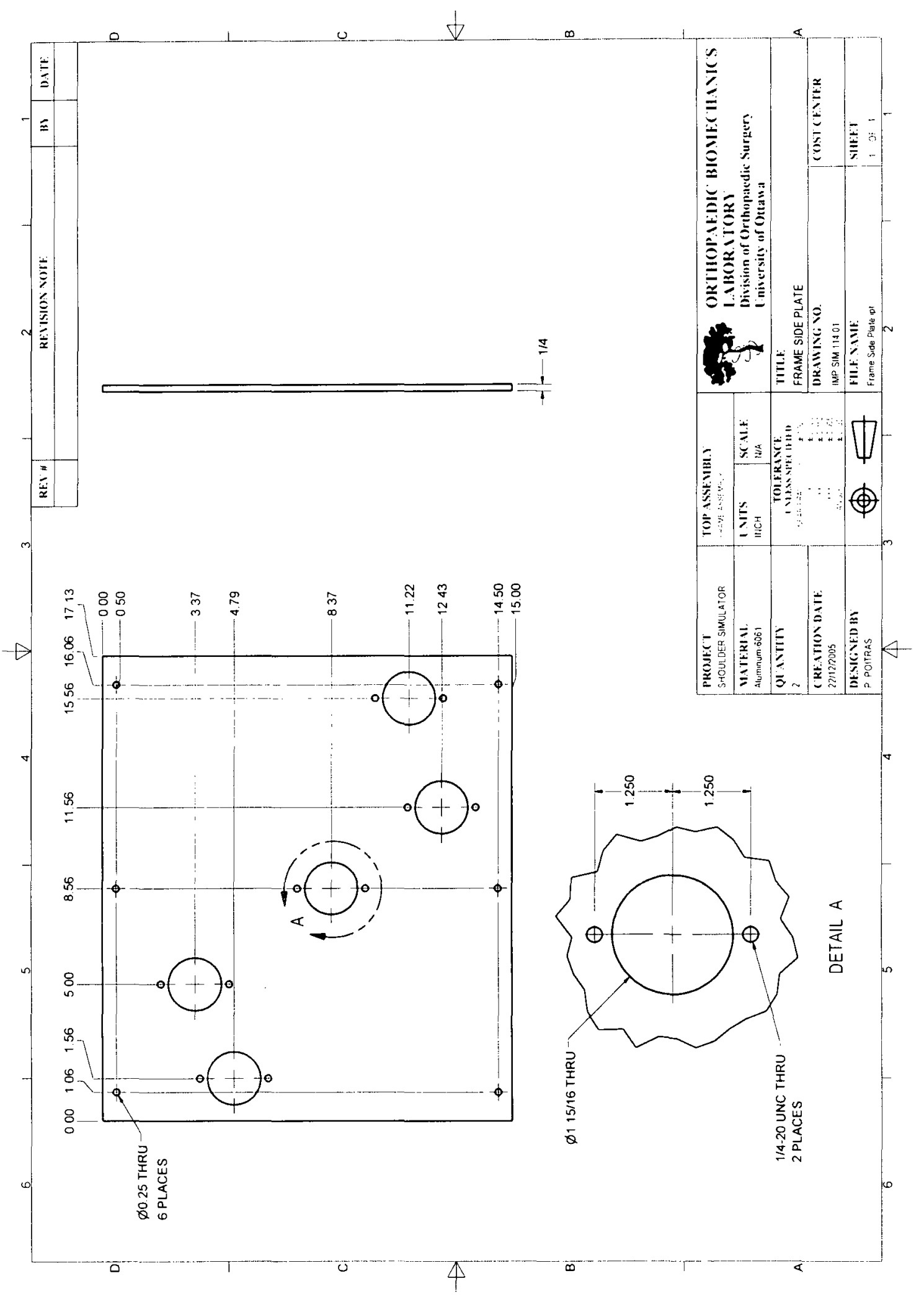




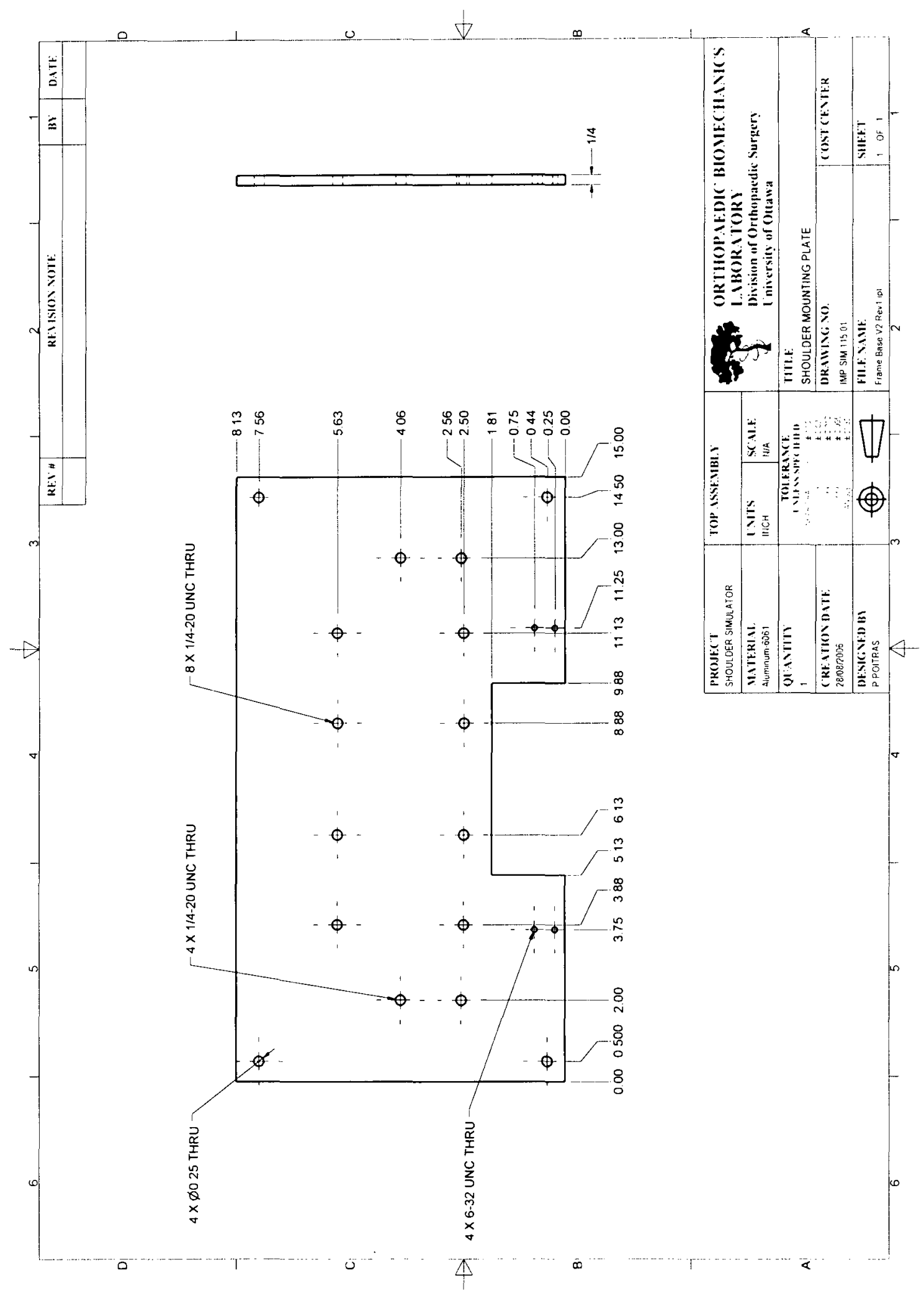




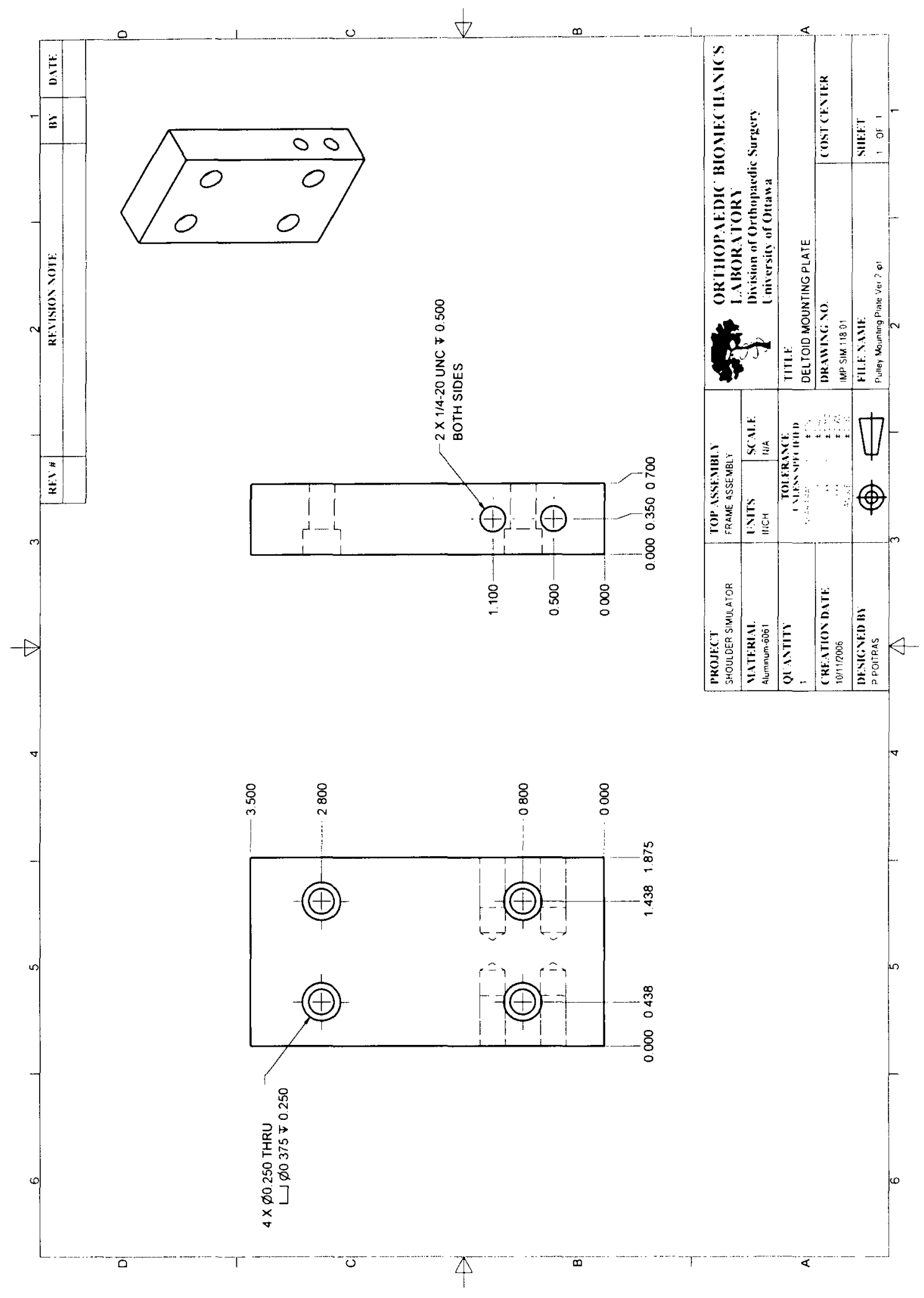




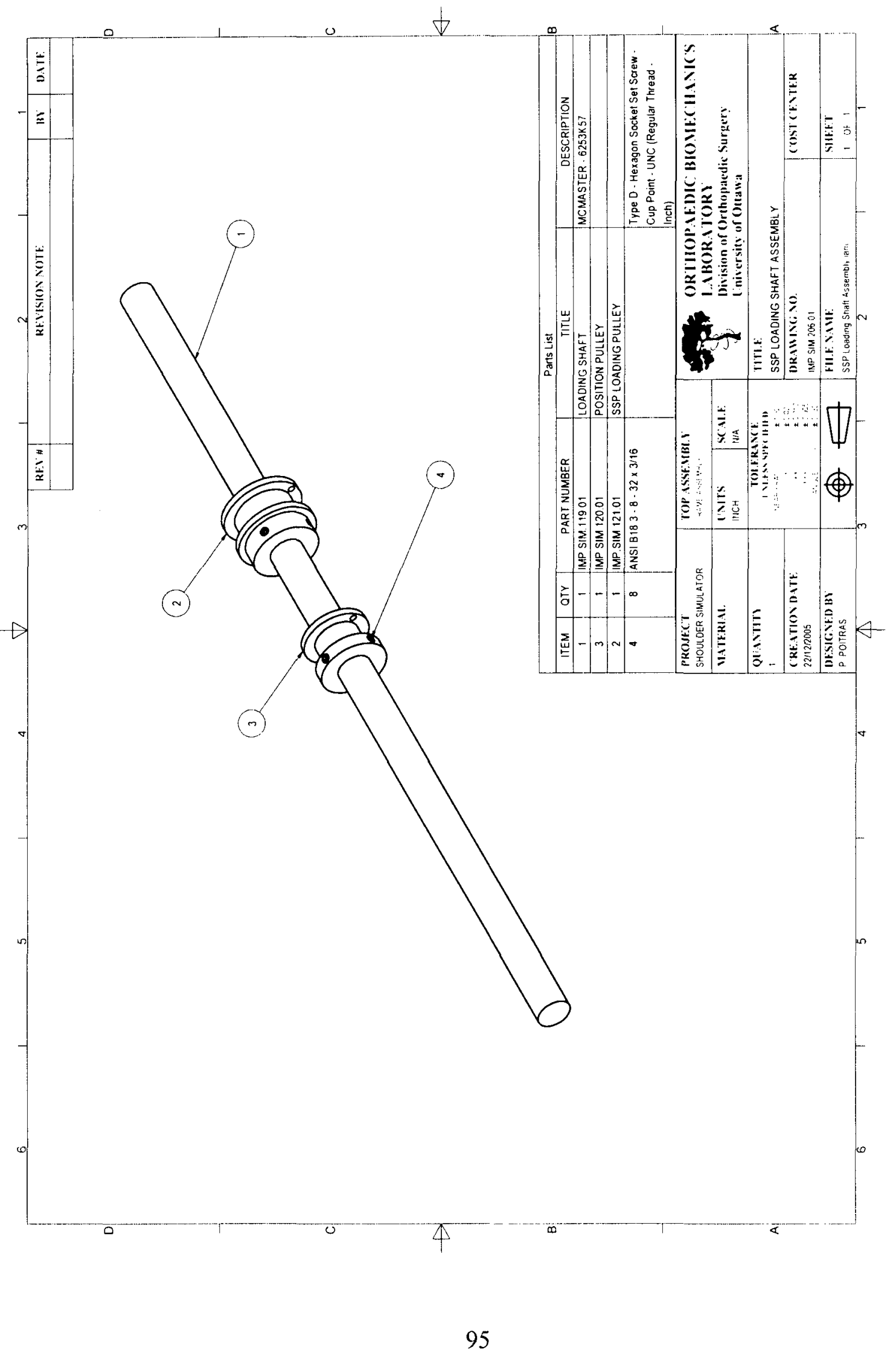




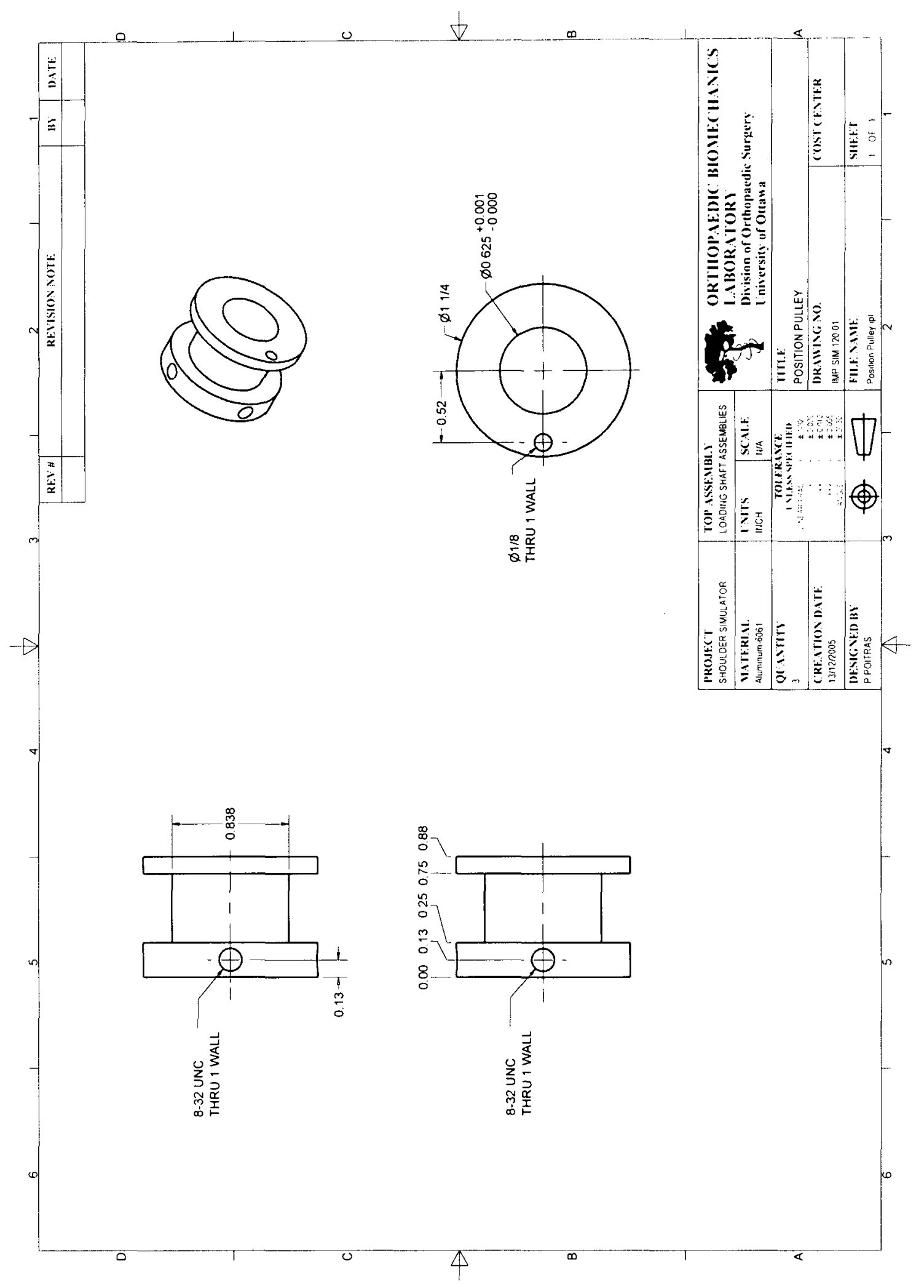




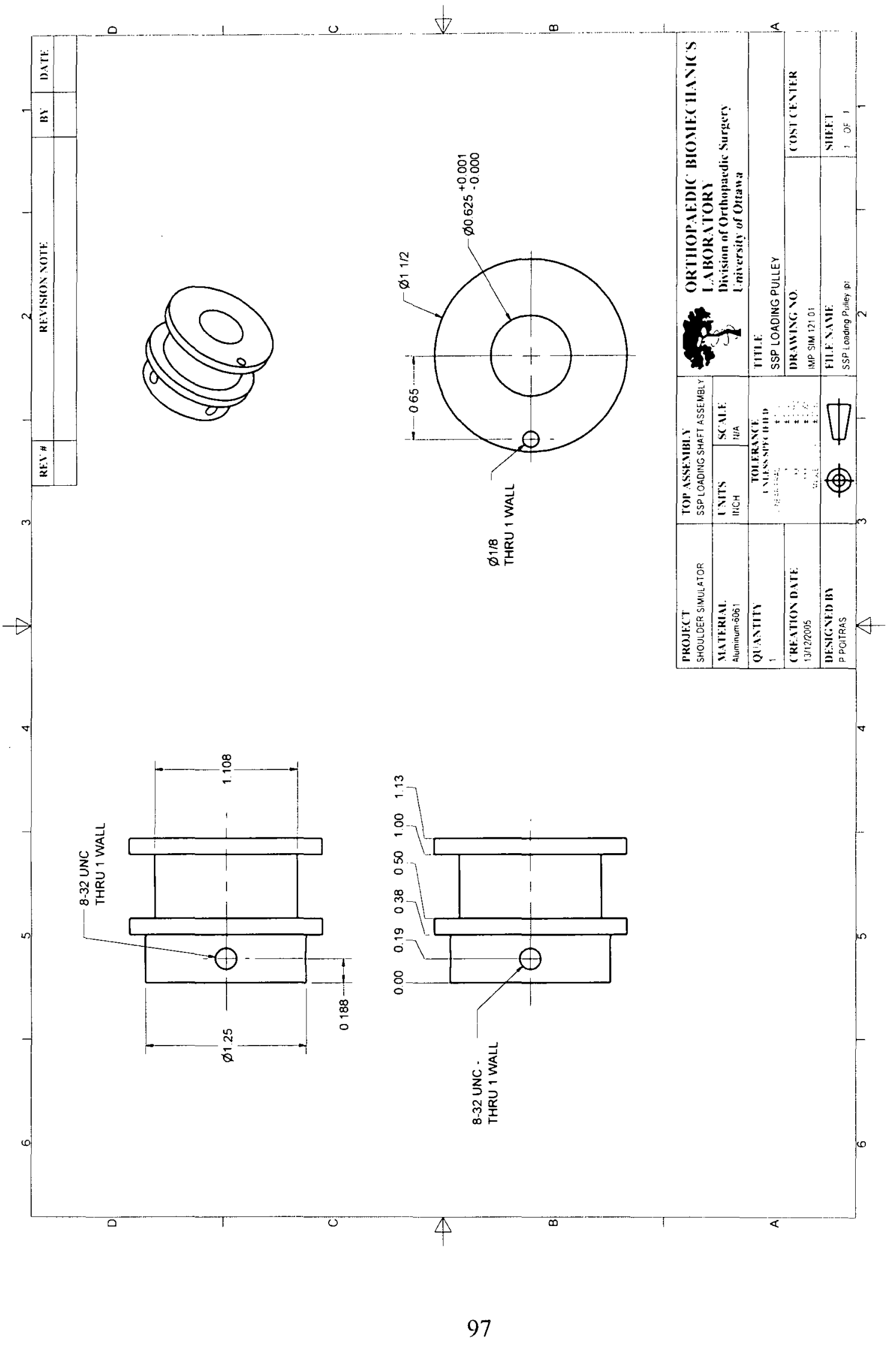




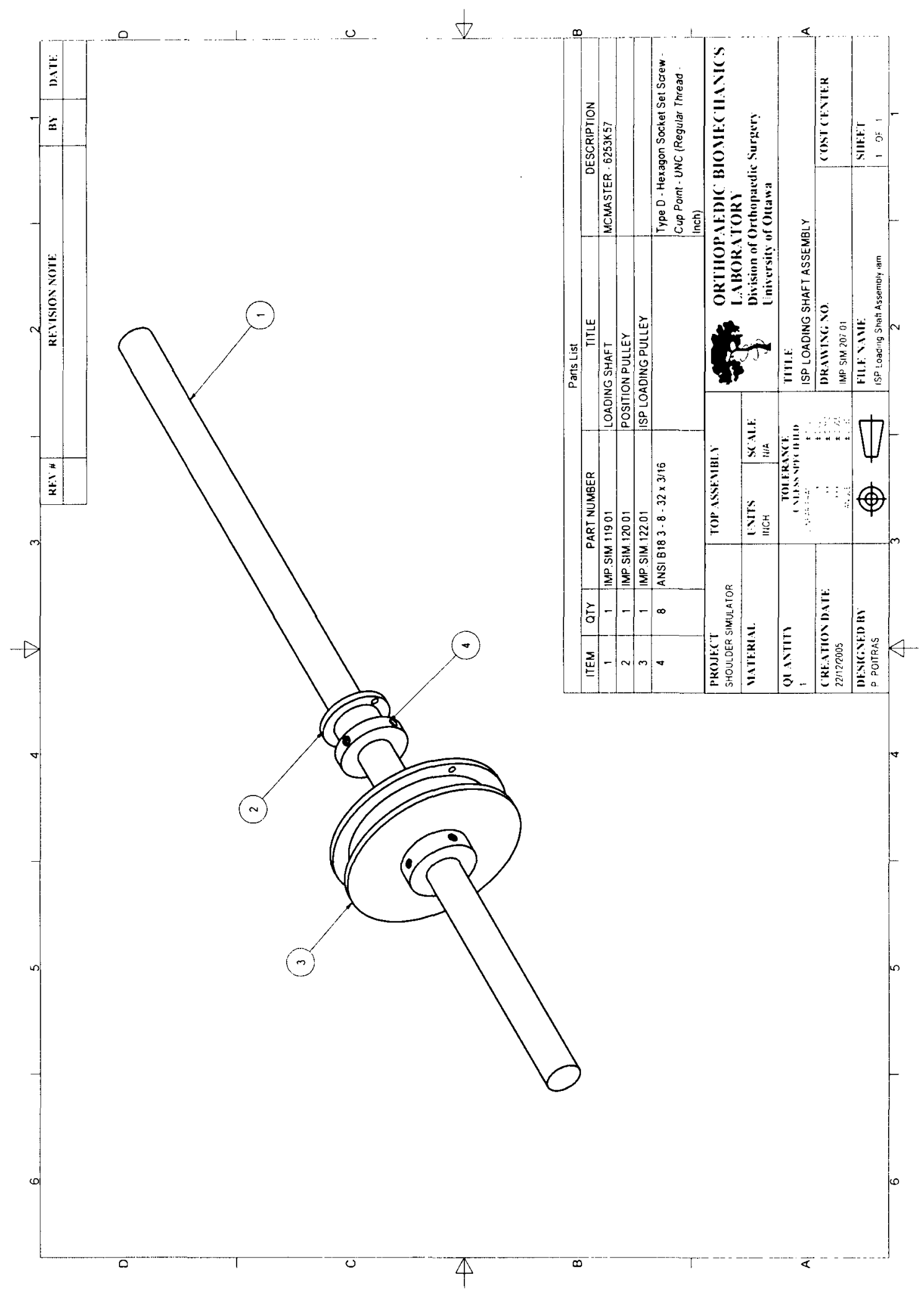




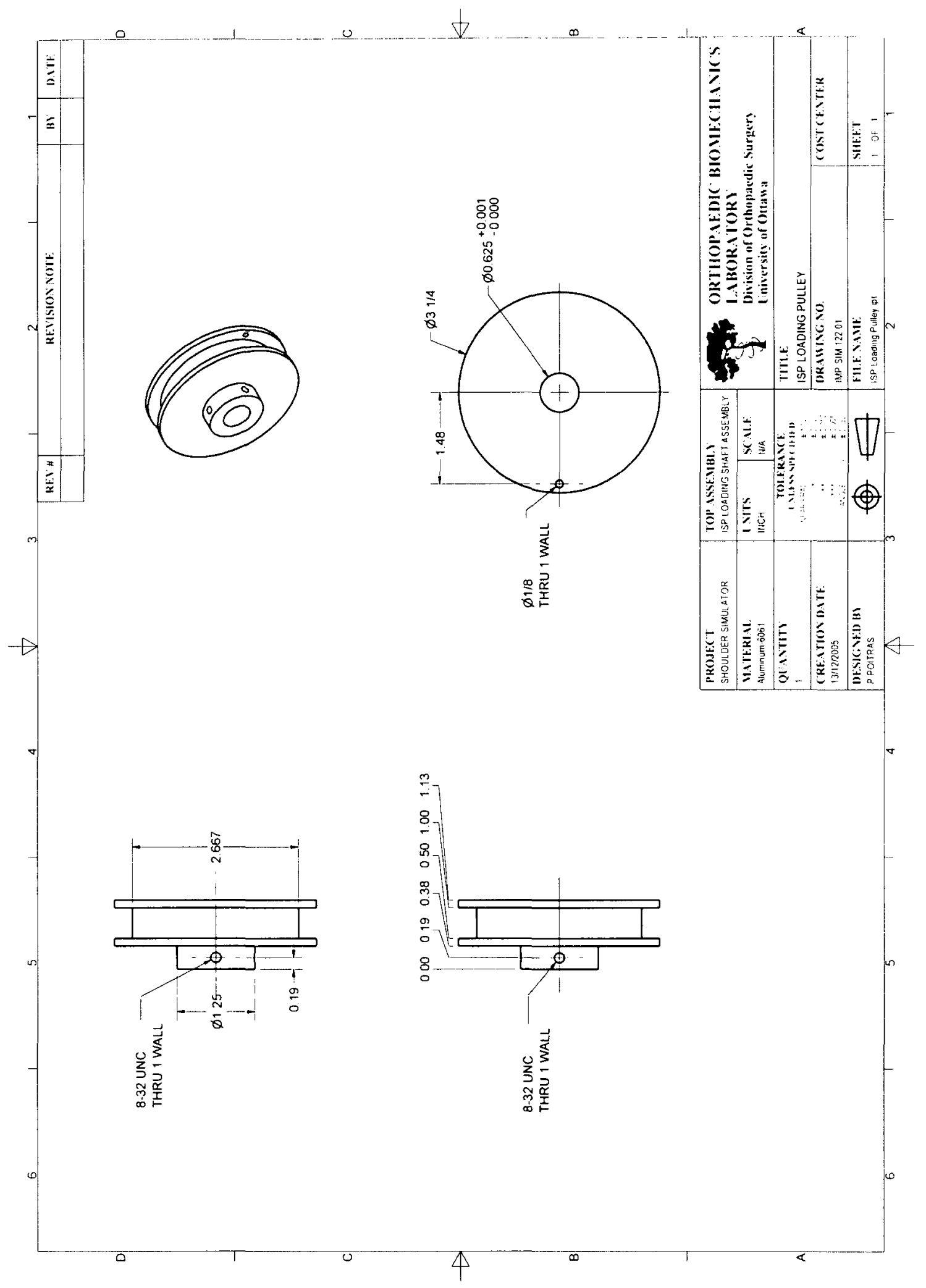




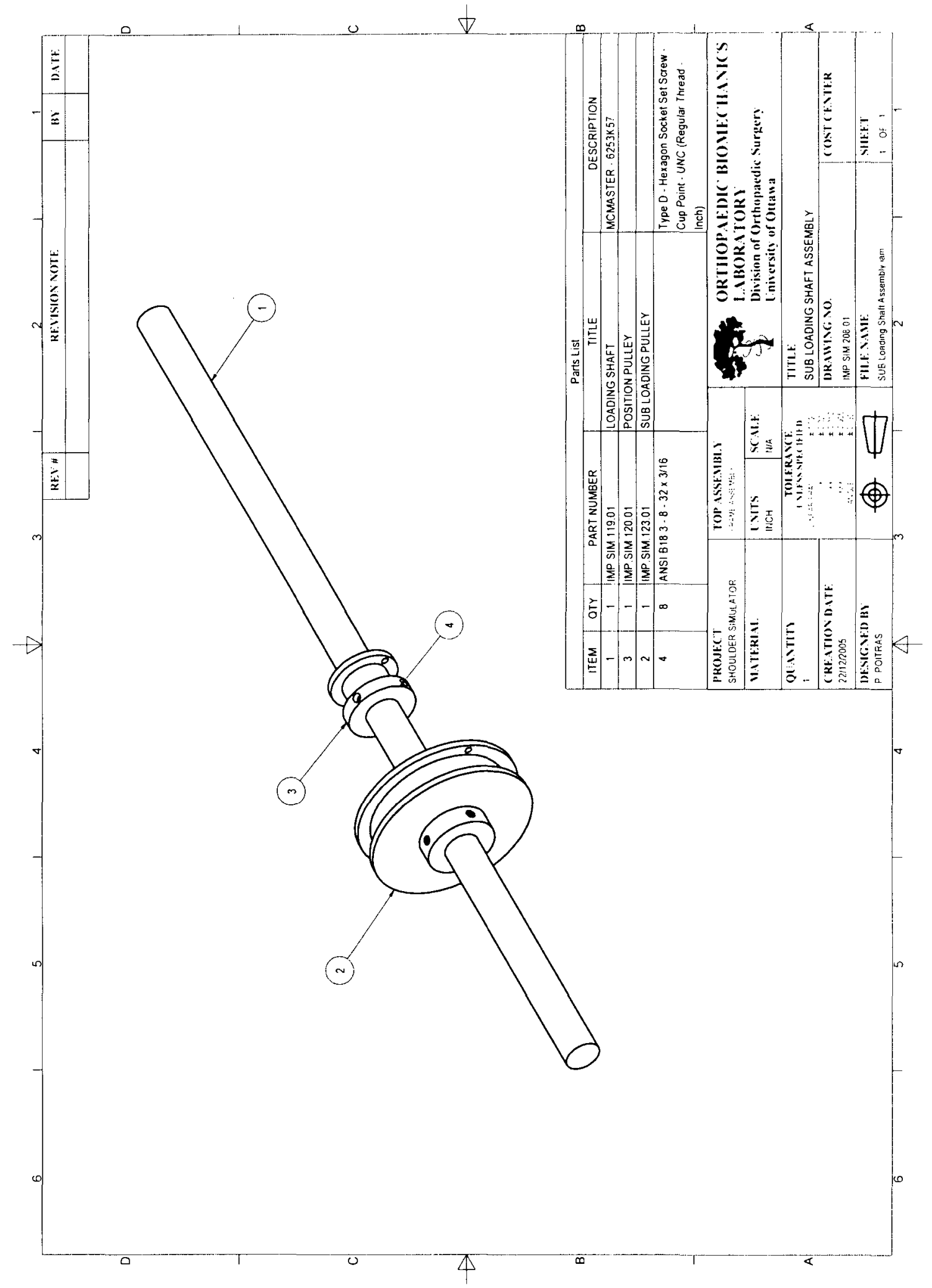




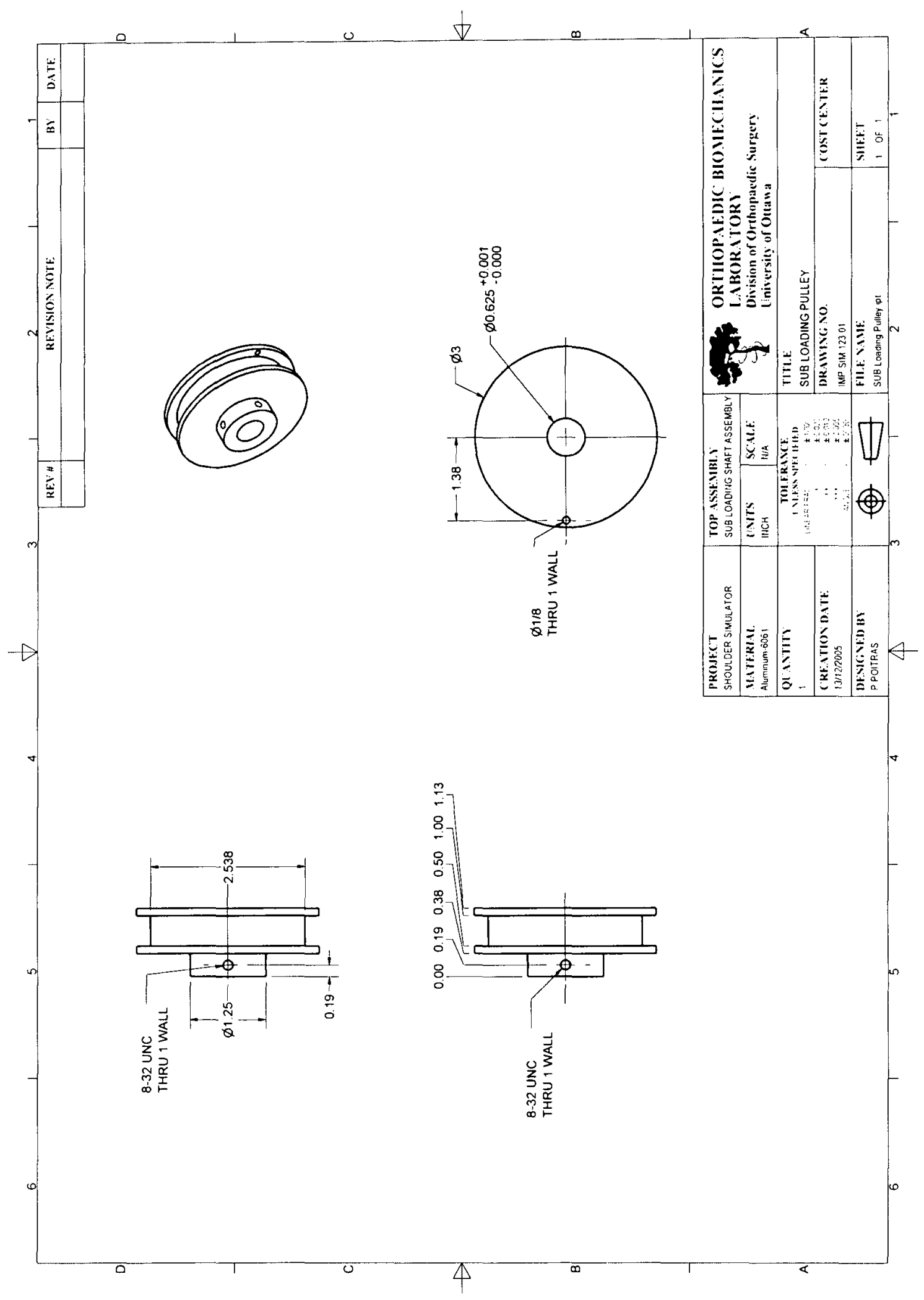




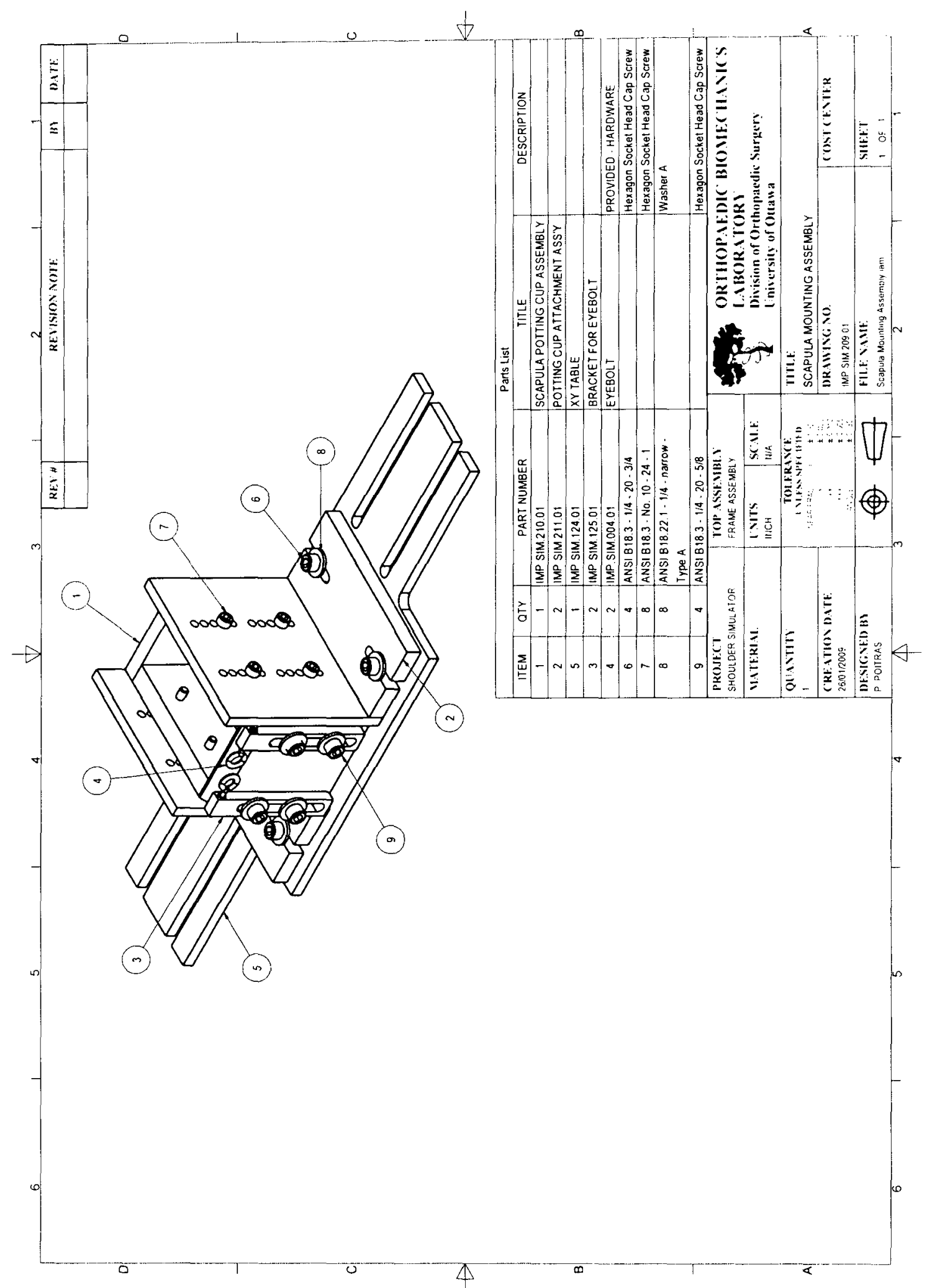




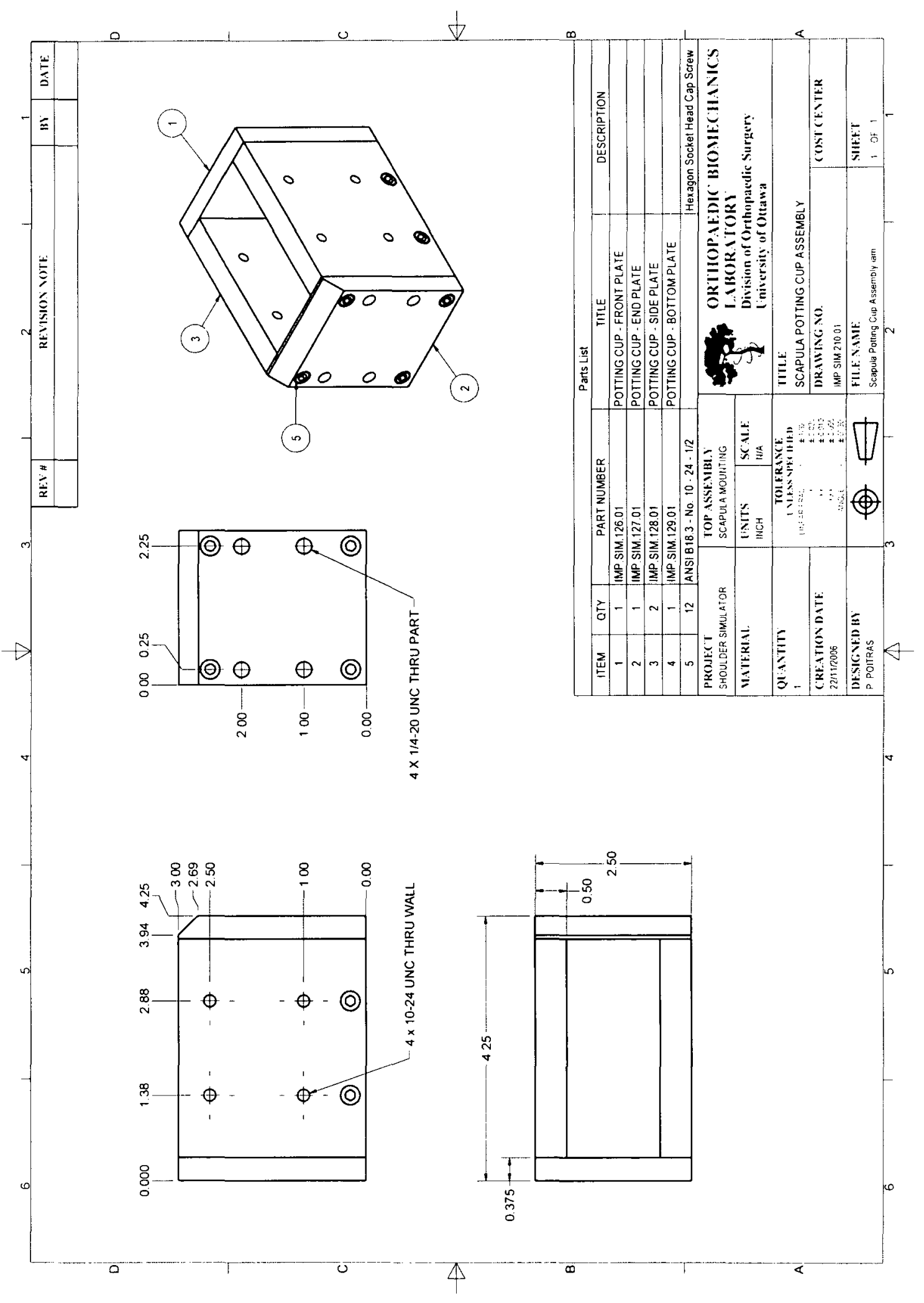




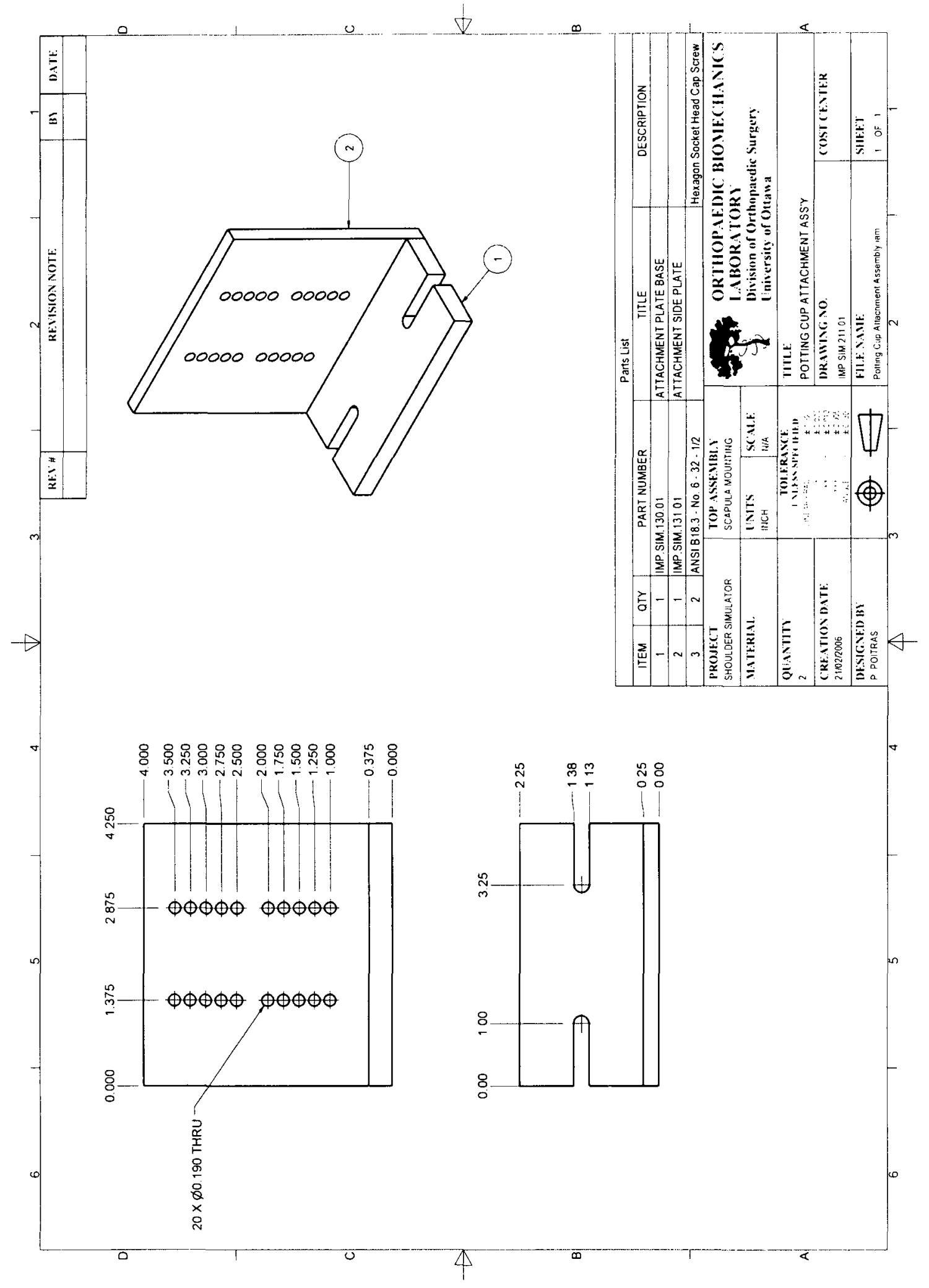




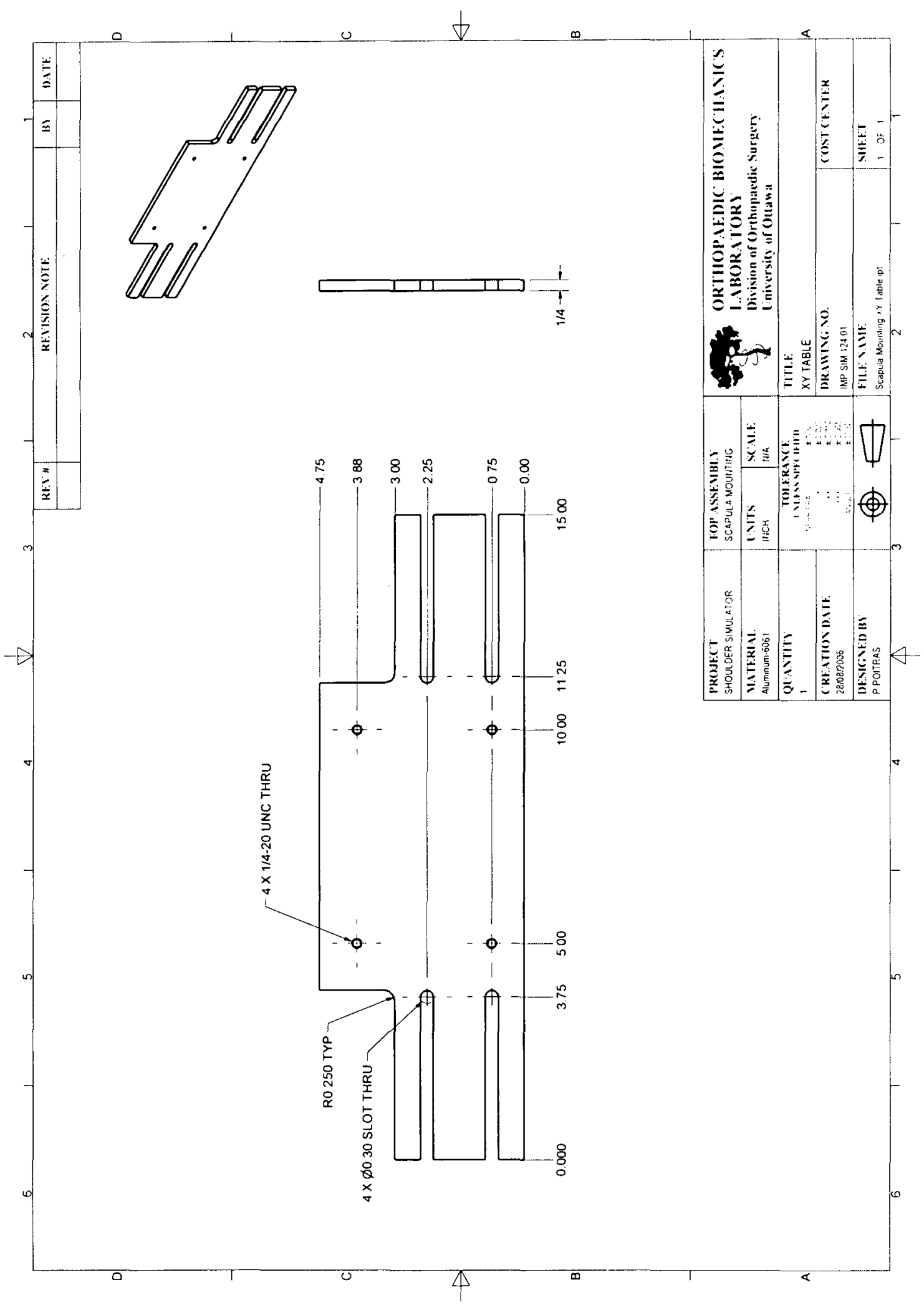




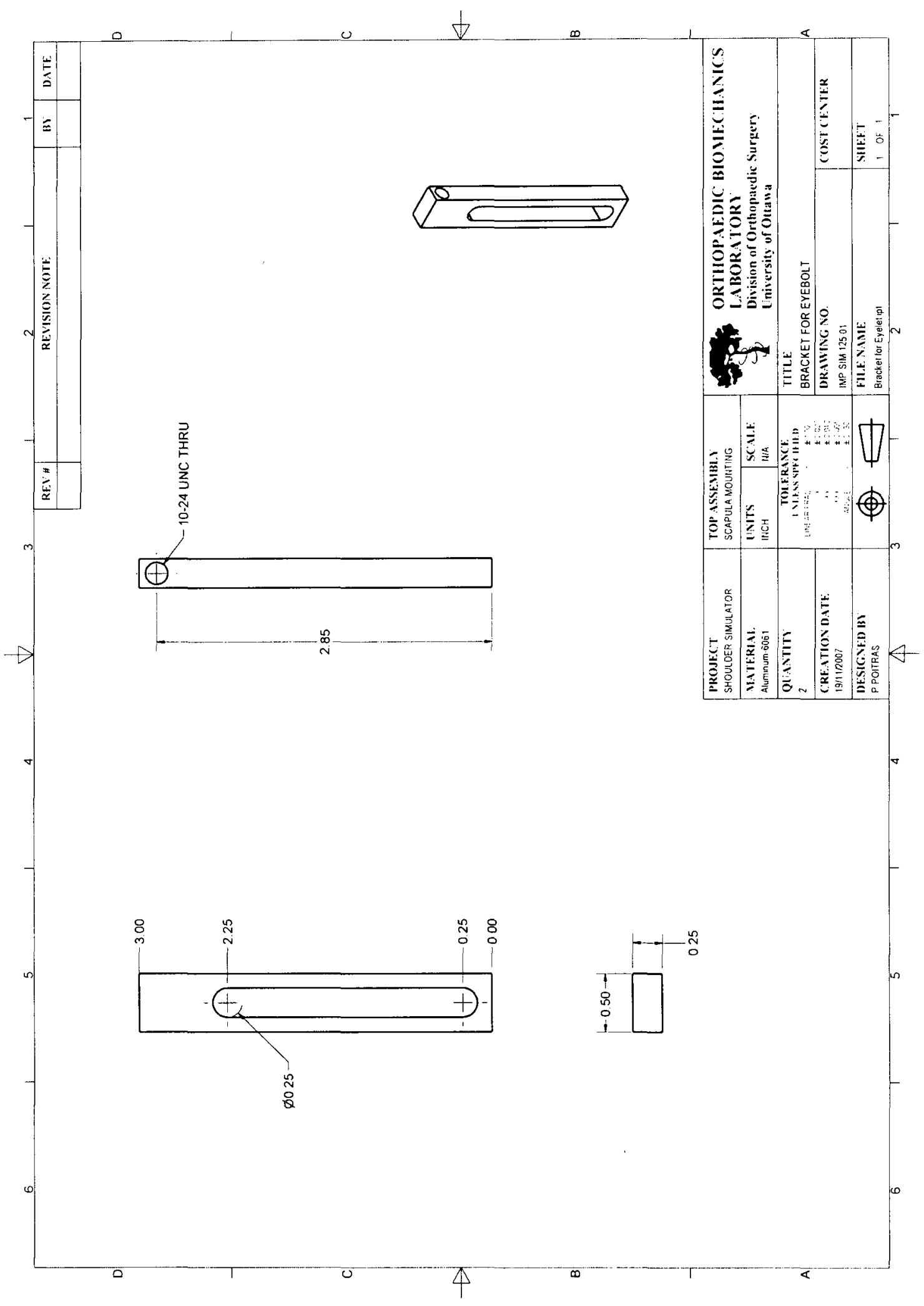




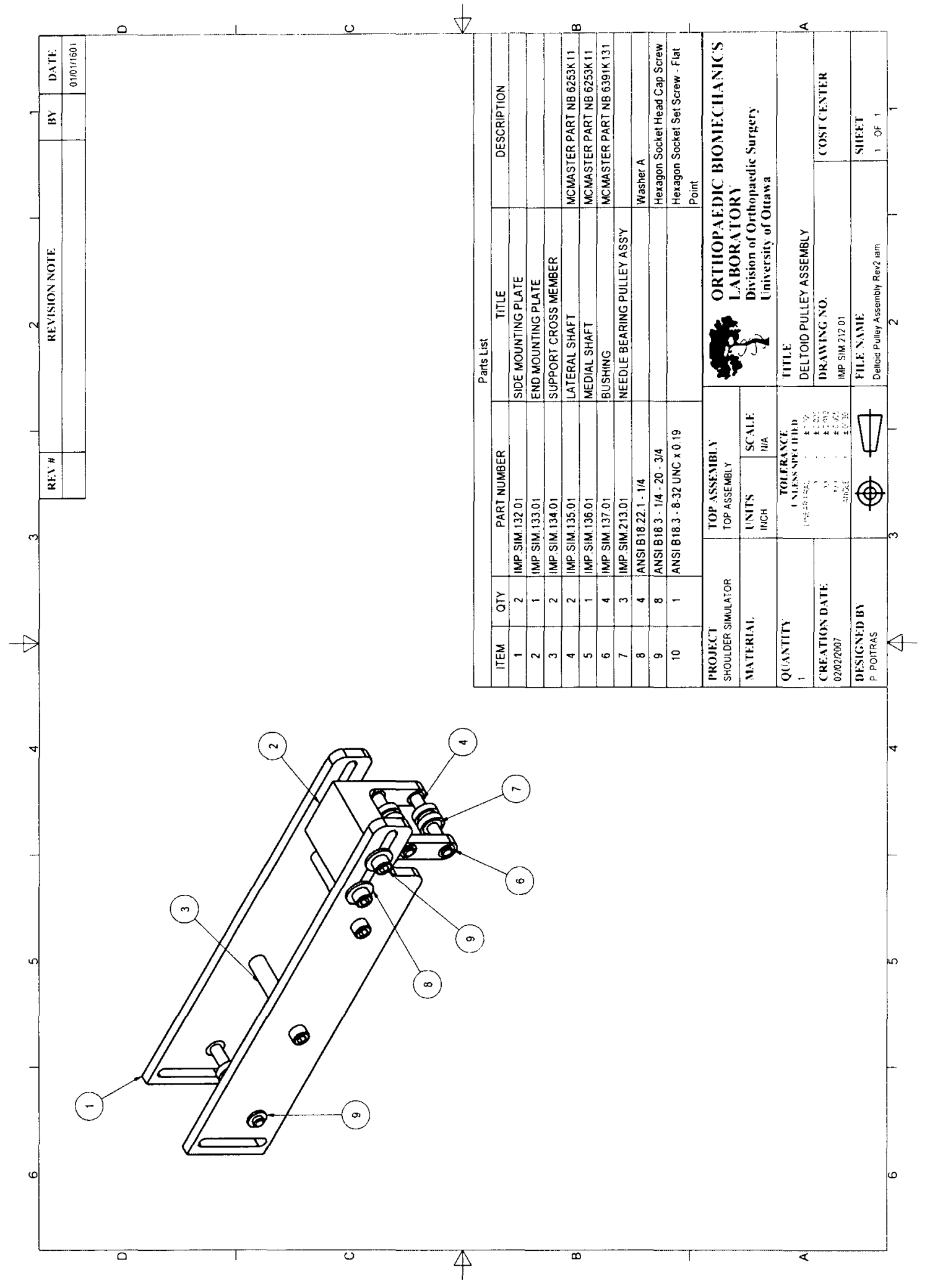




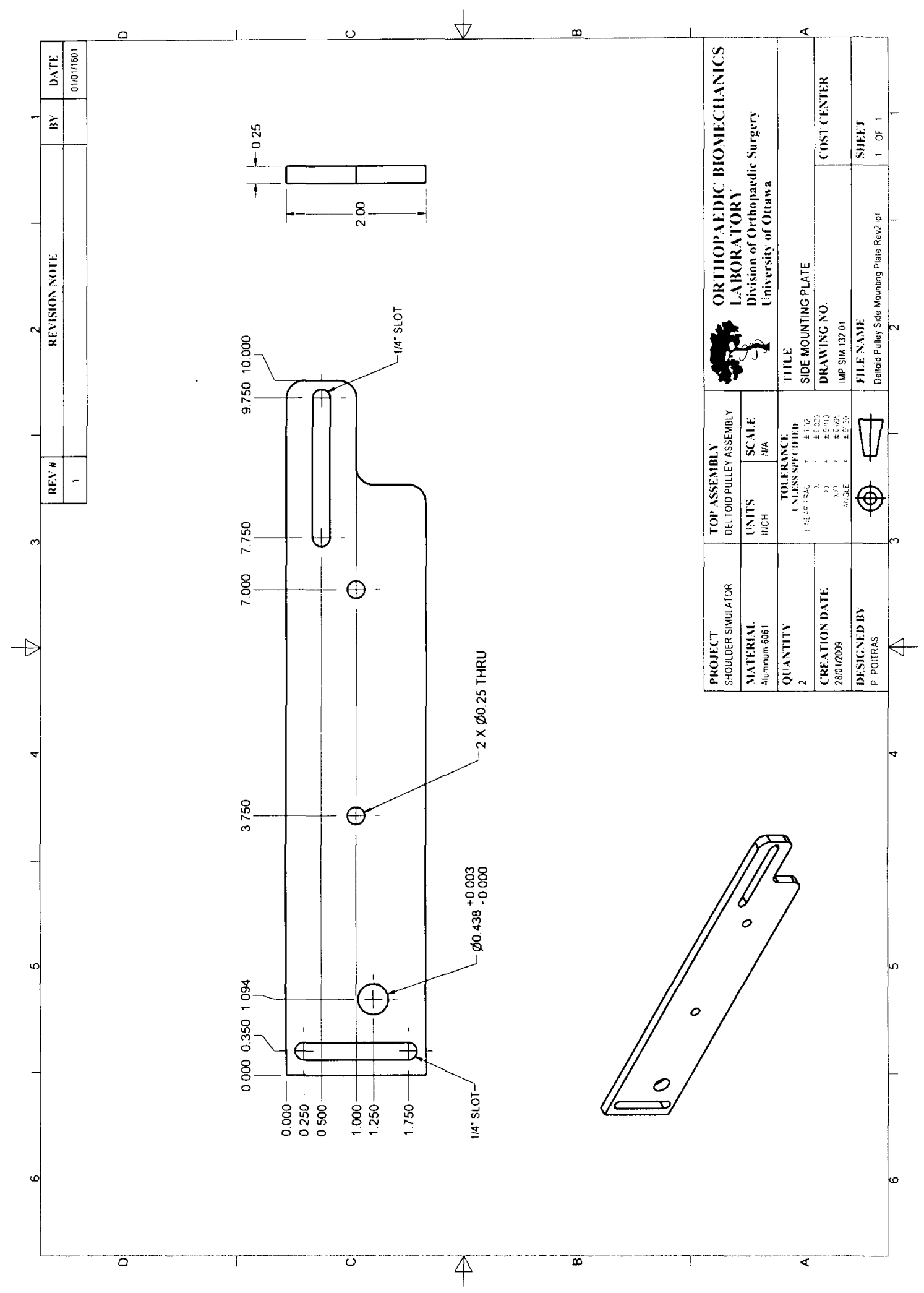




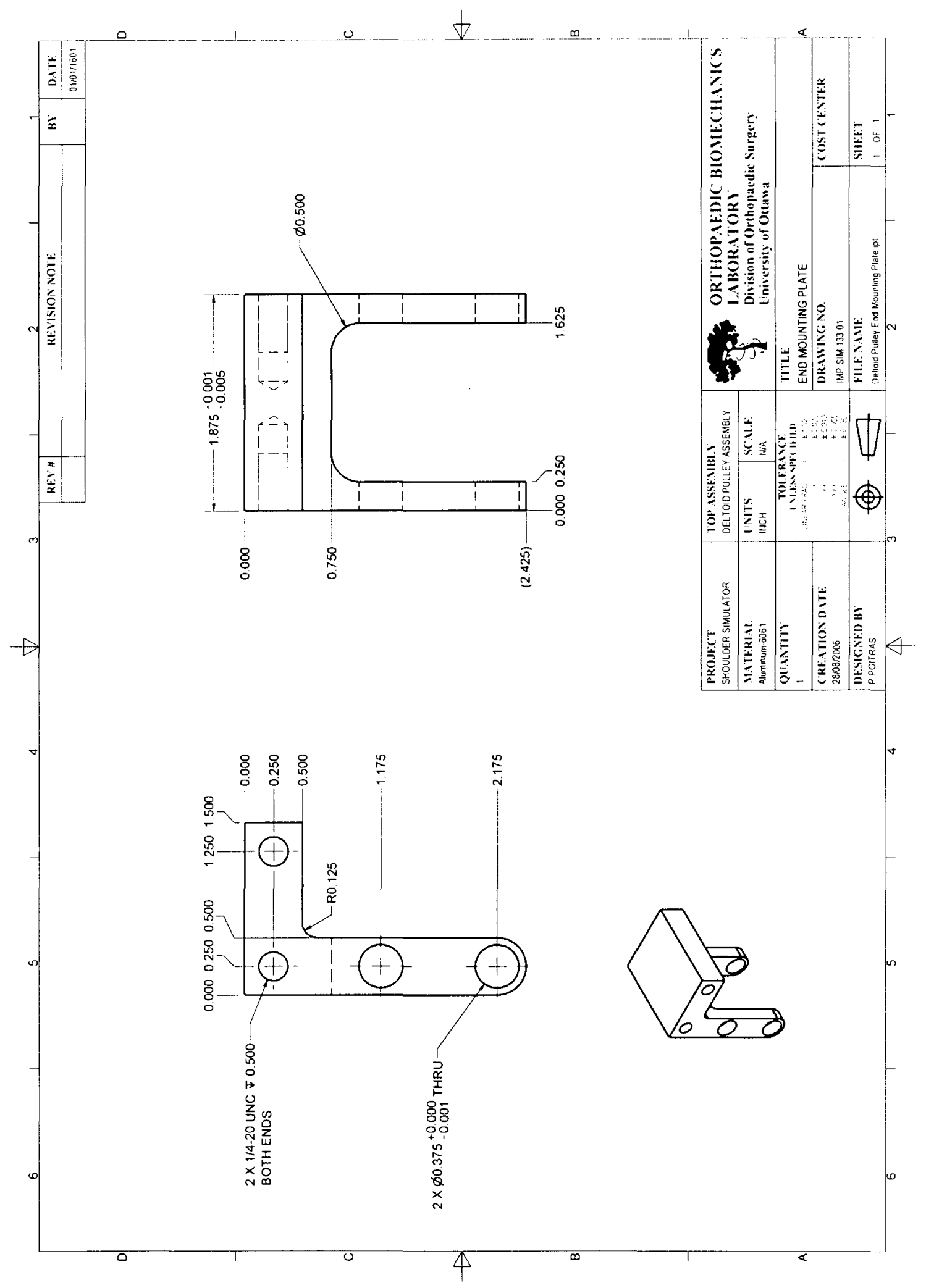




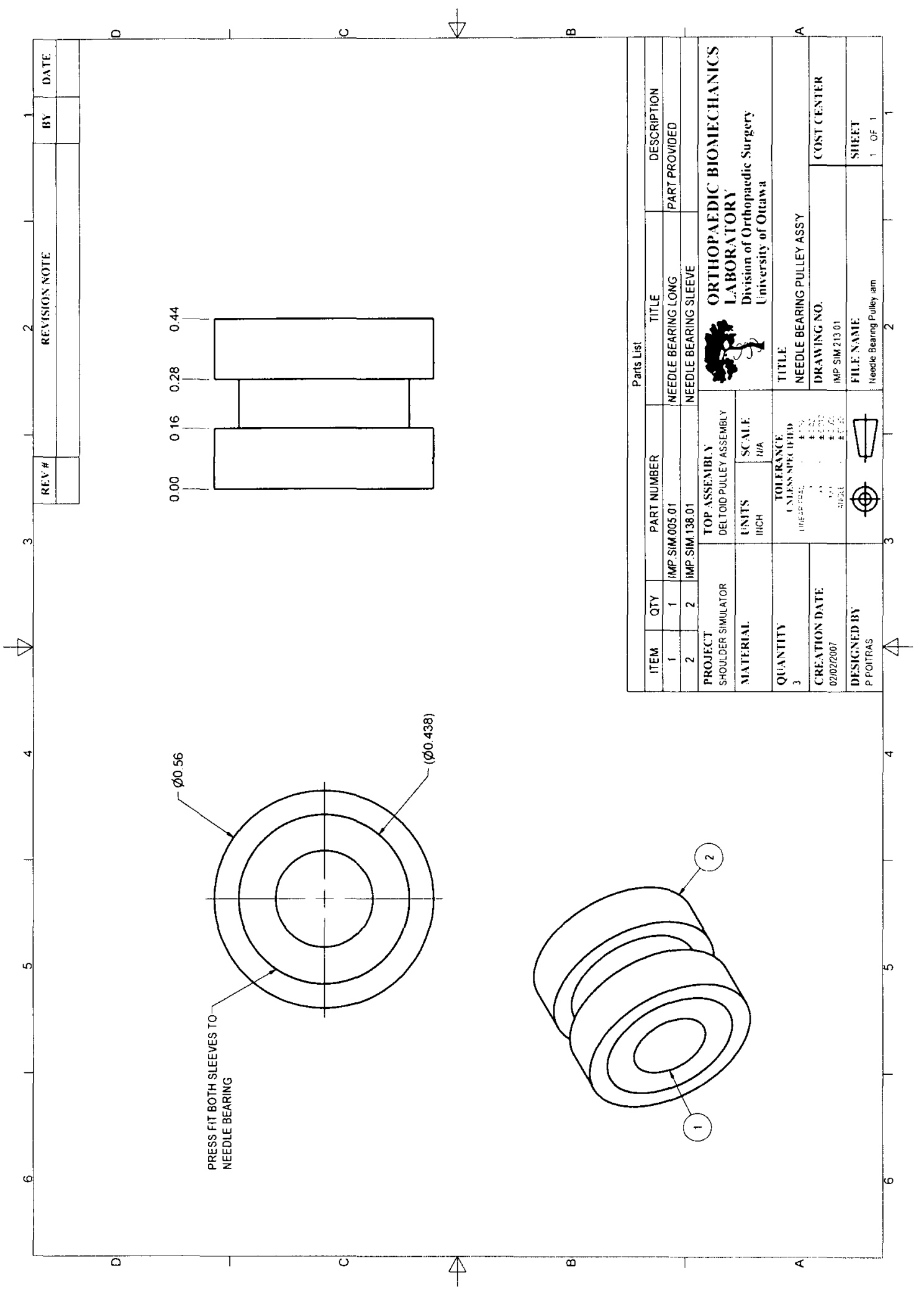




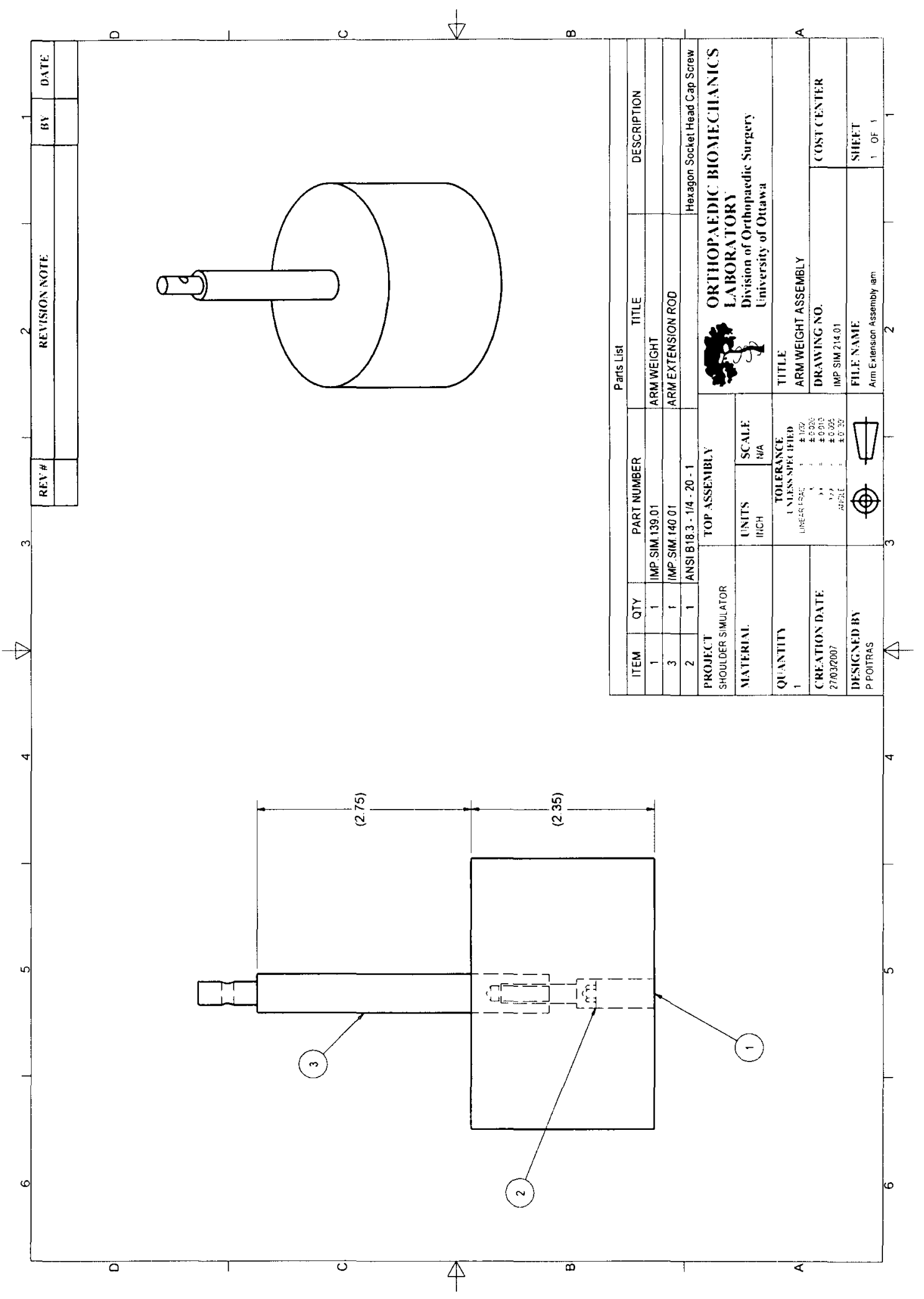




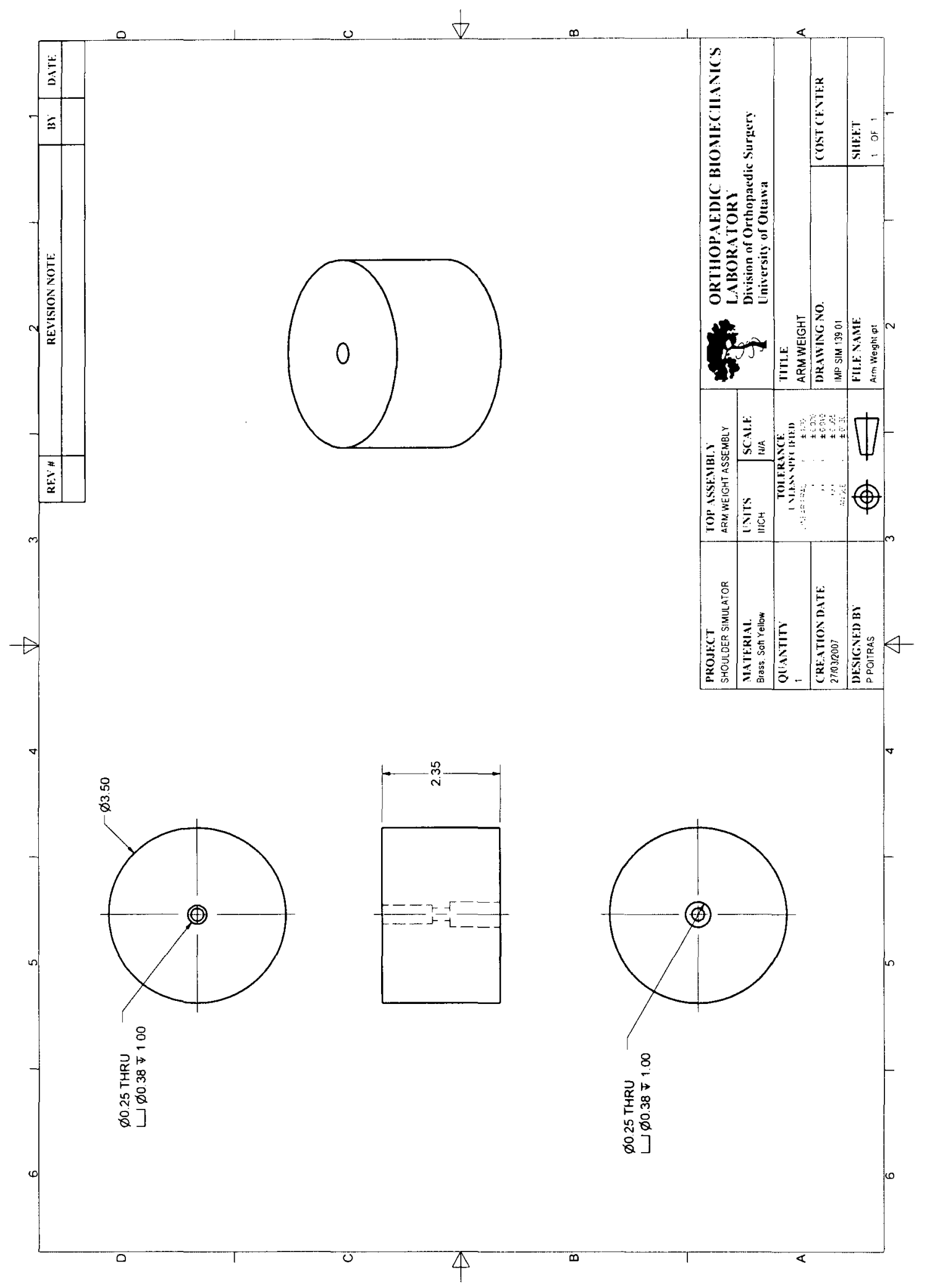




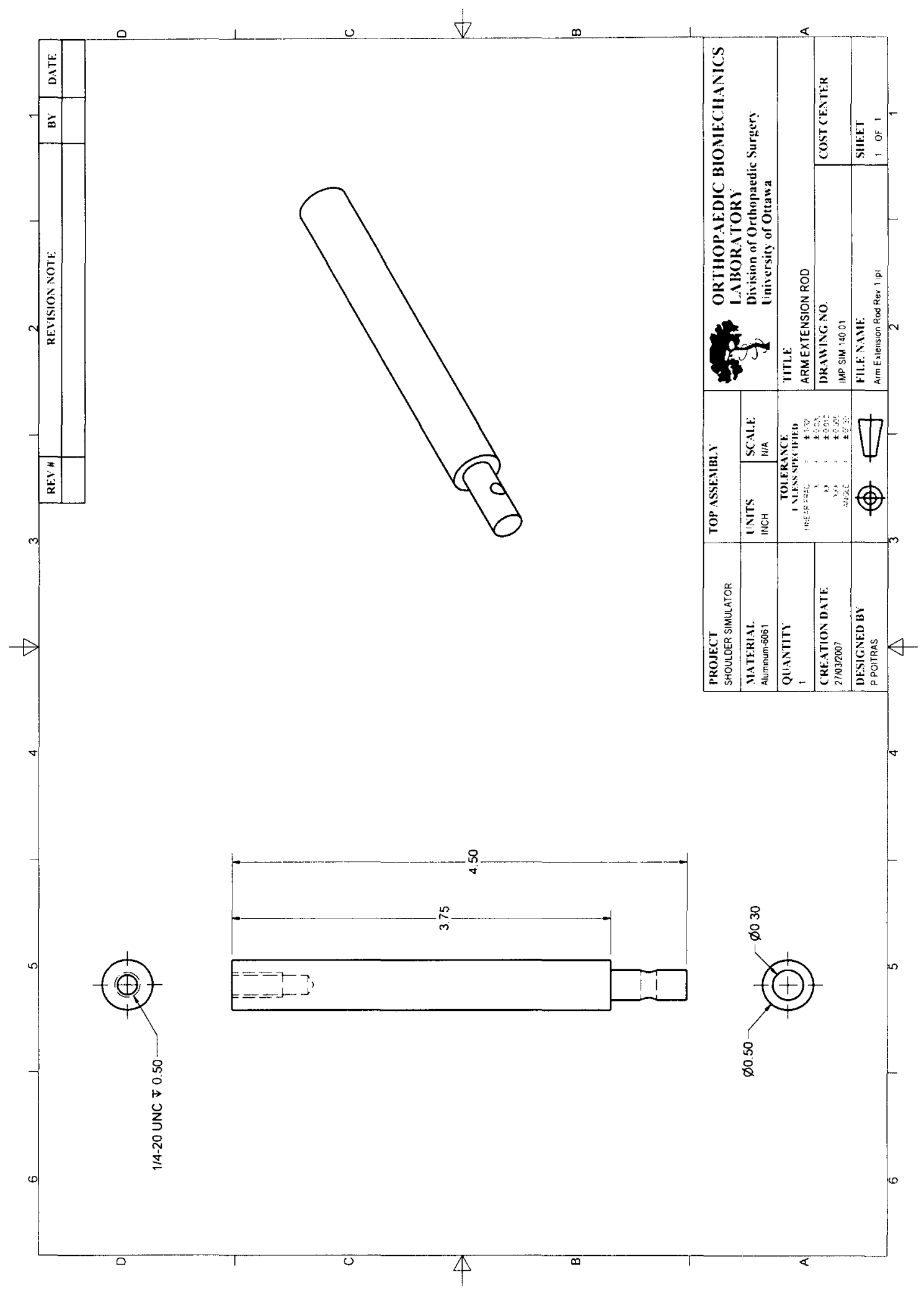




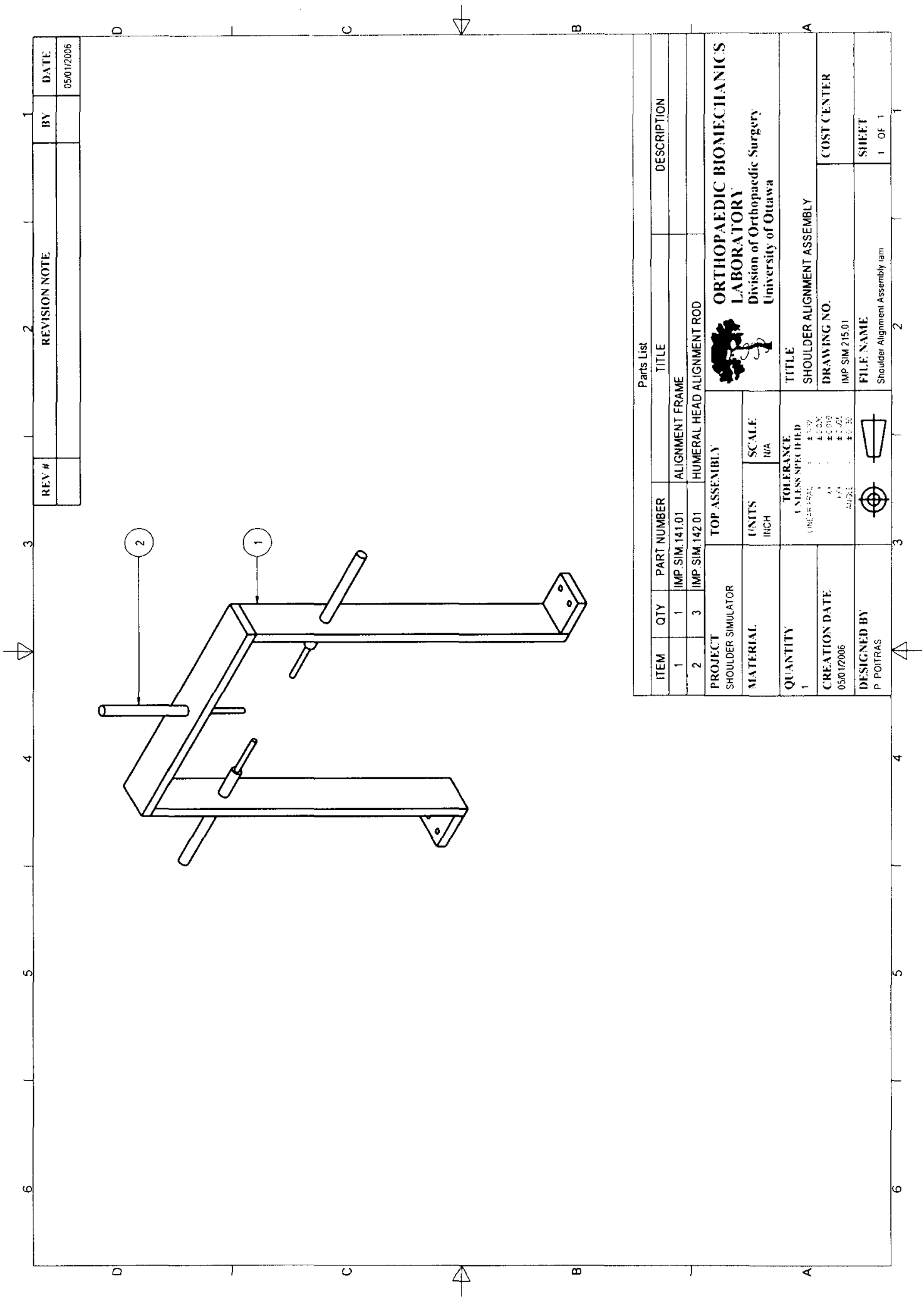




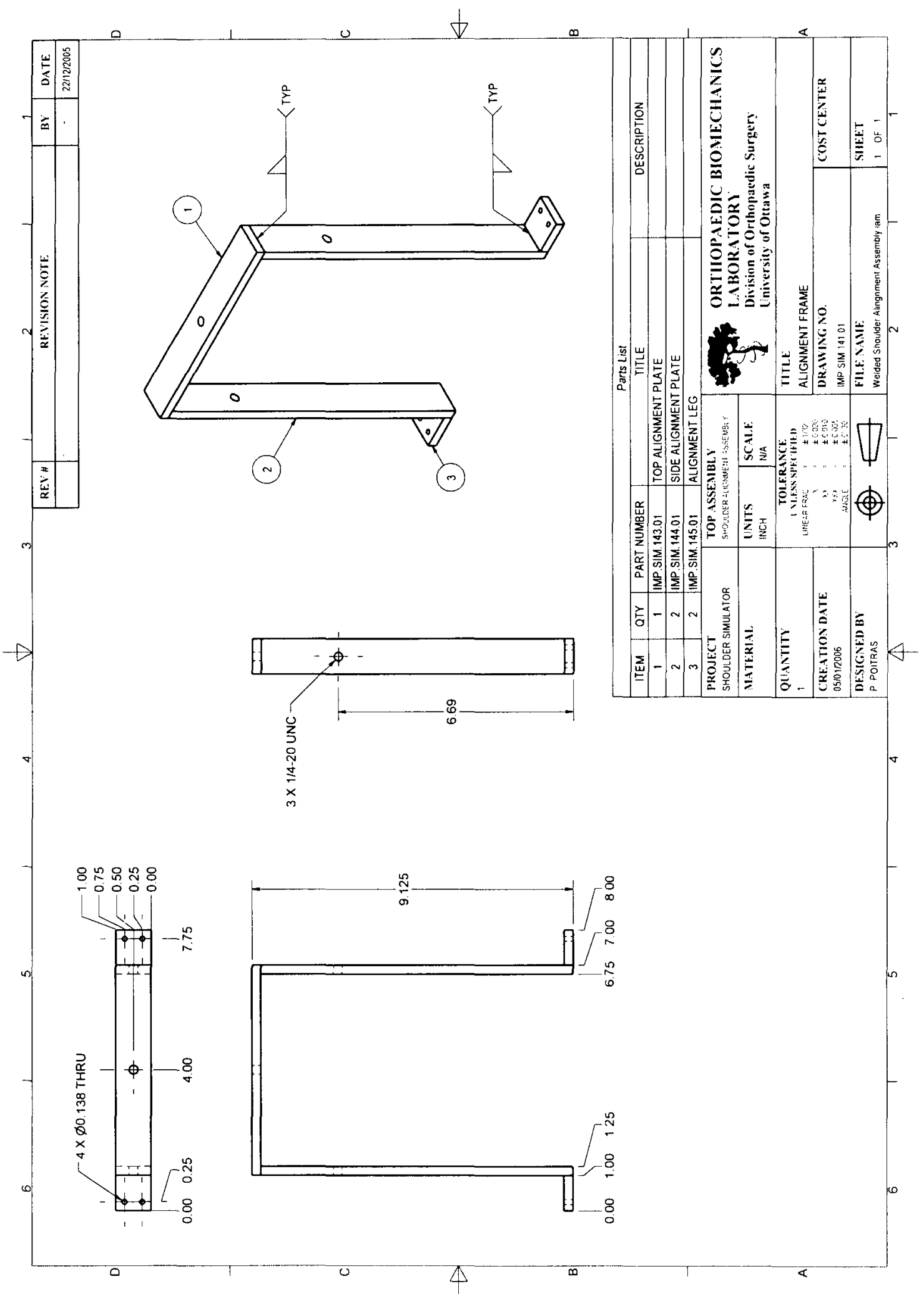


Appendix C.2: Polaris Passive Marker Tools - Engineering Drawings

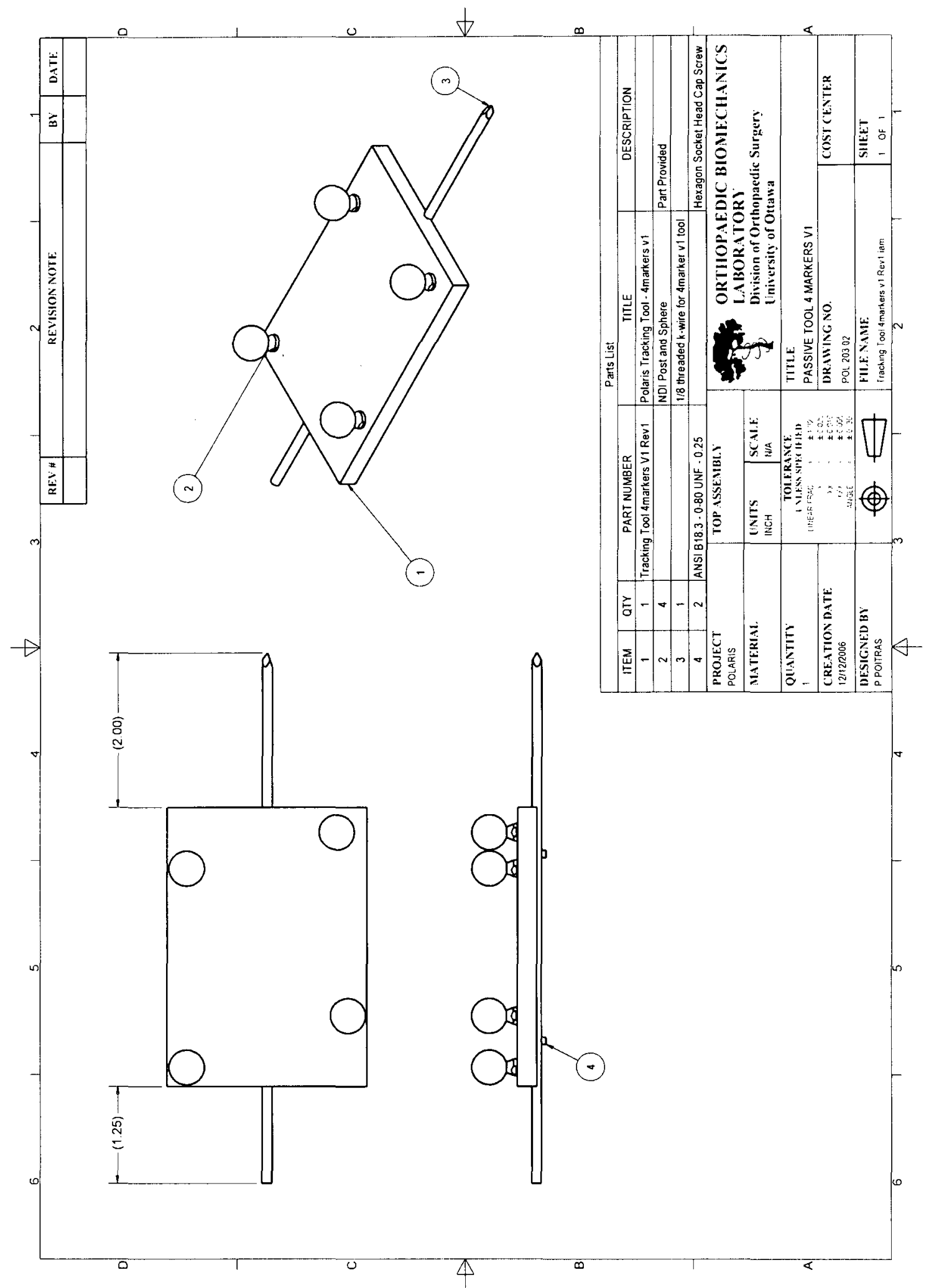




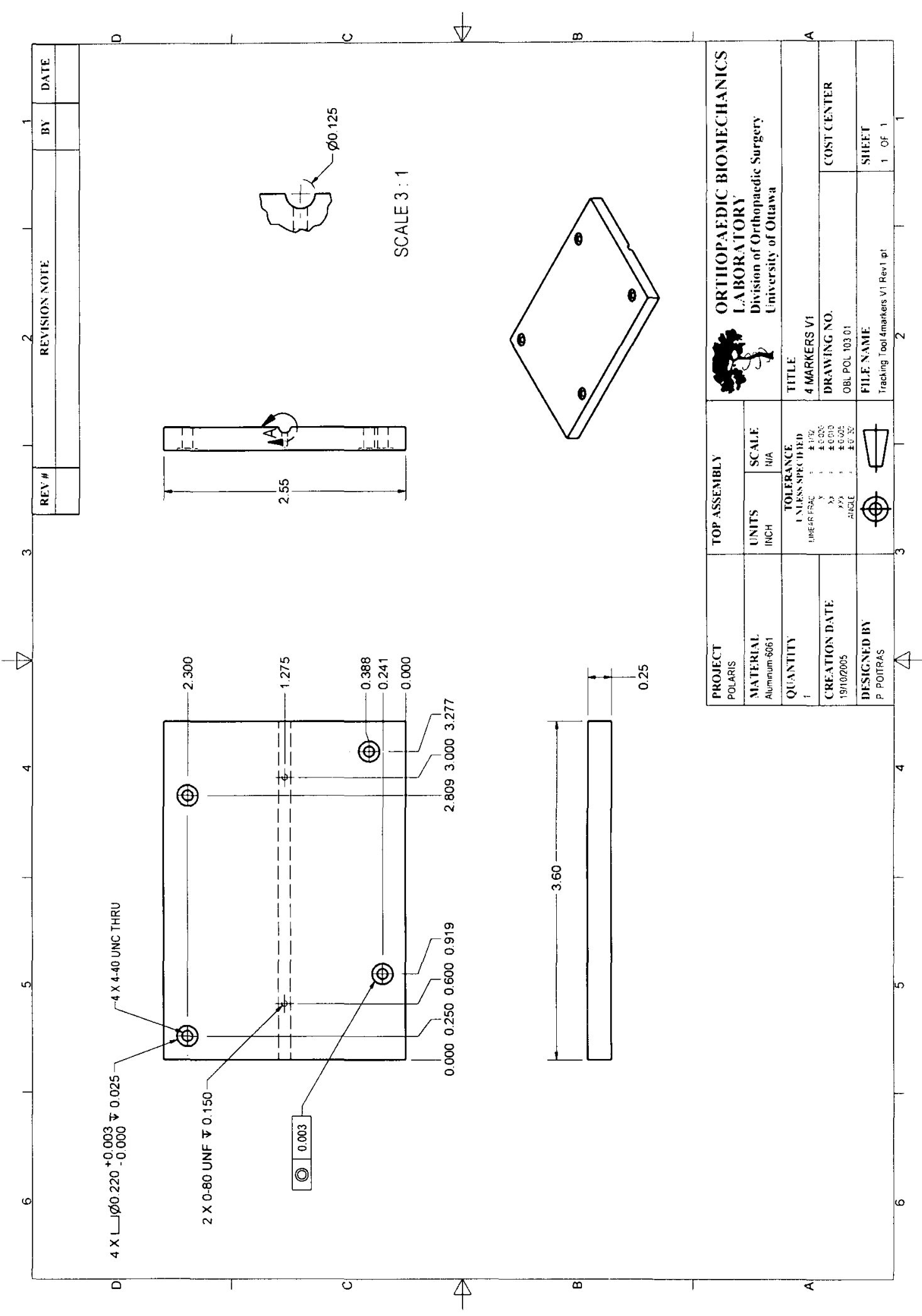




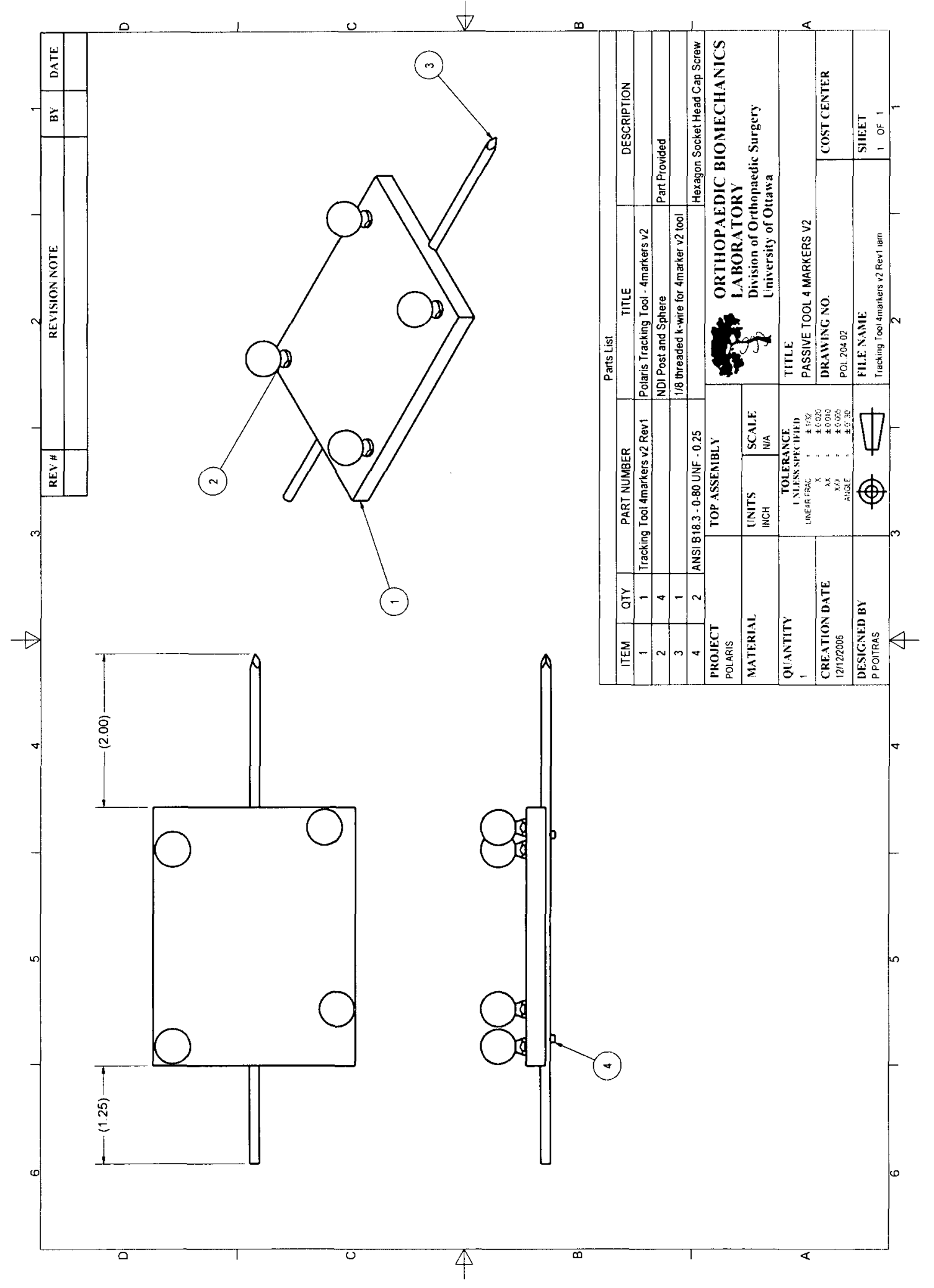




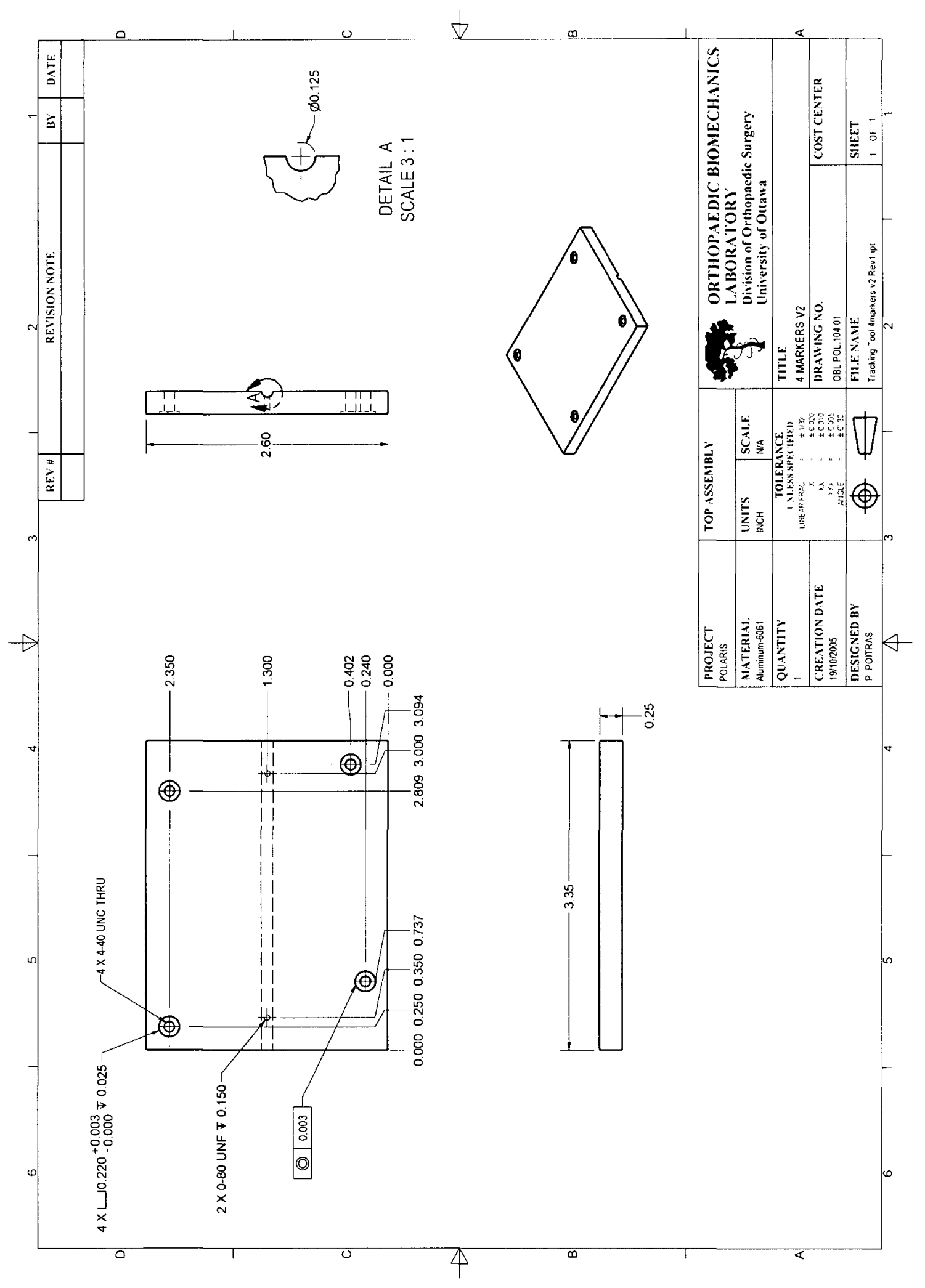

